\title{
OPERADS, CONFIGURATION SPACES AND QUANTIZATION
}

\author{
S.A. MERKULOV
}

\begin{abstract}
We review several well-known operads of compactified configuration spaces and construct several new such operads, $\bar{C}$, in the category of smooth manifolds with corners whose complexes of fundamental chains give us (i) the 2-coloured operad of $A_{\infty}$-algebras and their homotopy morphisms, (ii) the 2-coloured operad of $L_{\infty}$ algebras and their homotopy morphisms, and (iii) the 4-coloured operad of open-closed homotopy algebras and their homotopy morphisms.

Two gadgets - a (coloured) operad of Feynman graphs and a de Rham field theory on $\bar{C}$ - are introduced and used to construct quantized representations of the (fundamental) chain operad of $\bar{C}$ which are given by Feynman type sums over graphs and depend on choices of propagators.

Mathematics Subject Classifications (2000). 53D55, 16E40, 18G55, 58A50.

KEY WORDS. Poisson geometry, homotopy Lie algebras, configuration spaces.
\end{abstract}

\section{CONTENTs}

\section{Introduction}

2. Associahedra as compactified configuration spaces of points on the real line

2.1. Stasheff's associahedra and configuration spaces

2.2. Smooth structure on $\bar{C}_{n}(\mathbb{R})$

2.3. Induced orientation on the boundary strata

2.4. An equivalent definition of $\bar{C} \cdot(\mathbb{R})$

3. Multiplihedra as compactified configuration spaces of points on the real line

3.1. The first configuration space model

3.2. Another configuration space model for $\operatorname{Mor}\left(\mathcal{A}_{\infty}\right)$

3.3. One more configuration space model for $\operatorname{Mor}\left(\mathcal{A}_{\infty}\right)$

3.4. Metric graphs and smooth structures on compactified configuration spaces

4. Kontsevich configuration spaces and open-closed homotopy algebras

4.1. Fulton-MacPherson compactification of points on the complex plane Ko2

4.2. Kontsevich's compactification of points in the upper half plane Ko2

4.3. Higher dimensional version of $C_{n, m}(\mathbb{H})$ and open-closed homotopy Lie algebras

5. Configuration space models for the 2-coloured operad of $L_{\infty}$-morphisms

5.1. The complex plane models

5.2. Upper half space models for $\operatorname{Mor}\left(L_{\infty}\right) \mathrm{Me} 2$

6. Configuration space model for the 4-coloured operad of OCHA morphsisms

6.1. New compactified configuration spaces $\widehat{\mathfrak{C}}_{n, m}(\mathbb{H})$

6.2. Theorem on the face complex of $\widehat{\mathfrak{C}}_{\bullet}, \bullet$

6.3. Semialgebraic structure on $\widehat{\mathfrak{C}}_{n, m}$

6.4. Higher dimensional version

7. Operads of Feynman graphs and their representations

7.1. An operad of Feynman graphs $\mathfrak{G}$

7.2. A class of representations of $\mathfrak{G}$

7.3. A class of representations of the 2-coloured operads $\mathfrak{G}^{\uparrow \downarrow}, \mathfrak{G}^{\uparrow}$ and $\mathfrak{G}^{\downarrow}$

8. De Rham field theories on configuration spaces and quantization

8.1. Completed tensor product of de Rham algebras

8.2. De Rham field theory on $\bar{C}\left(\mathbb{R}^{d}\right)$

8.3. De Rham field theories on a class of free topological operads

9. Examples of quantized representations of operads of Feynman diagrams 43

9.1. Propagators on $\bar{C}\left(\mathbb{H}^{d}\right)$

9.2. Examples of propagators

9.3. Deformation quantization of associative algebras of polyvector fields

9.4. Kontsevich's formality maps 
9.5. Deformation quantization of the Schouten bracket

10. Towards the theory of open-closed morphisms of deformation quantizations

10.1. De Rham field theory on the first model of $\widehat{\mathfrak{C}}_{\bullet}(\mathbb{C})$

10.2. On (auto)morphisms of deformation quantizations

Appendix A. Operads and coloured operads BM, GJ, GK, LV]

A.1. Trees

A.2. $\mathcal{S}$-modules

A.3. Definitions of an operad

A.4. Coloured operads

A.5. Coloured operads of transformation type

References

\section{Introduction}

1.1. Configuration spaces. This paper is inspired by Kontsevich's proof [Ko2] of his celebrated formality theorem. A central role in that proof is played by a 2-coloured operad of compactified configuration spaces, $\bar{C}(\mathbb{H})=\bar{C} \bullet(\mathbb{C}) \sqcup \bar{C} \bullet, \bullet(\mathbb{H})$, whose associated operad of fundamental chains, $\mathcal{F C}$ hains $(\bar{C}(\mathbb{H}))$, was termed in KaSt] an operad, $\mathcal{O C}_{\infty}$, of open-closed homotopy algebras.

We review in this paper the operad $\bar{C}(\mathbb{H})$, its lower and higher dimensional versions, and also construct several new operads, $\bar{C}$, of compactified configuration spaces in the category of smooth manifolds with corners (or in the category of semialgebraic manifolds) whose complexes of fundamental chains, $\mathcal{F C h a i n s}(\bar{C})$, give us

(i) the 2-coloured operad of $\mathcal{A}_{\infty}$-algebras and their homotopy morphisms, $\operatorname{Mor}\left(\mathcal{A}_{\infty}\right)$,

(ii) the 2-coloured operad of $\mathcal{L}_{\infty}$-algebras and their homotopy morphisms, $\operatorname{Mor}\left(\mathcal{L}_{\infty}\right)$, and

(iii) the 4-coloured operad of open-closed homotopy algebras and their homotopy morphisms, $\operatorname{Mor}\left(\mathcal{O C}_{\infty}\right)$.

An upper-half space model for $\operatorname{Mor}\left(\mathcal{L}_{\infty}\right)$ was studied earlier in [Me2]; in this paper we introduce several other configuration space models for this important 2-coloured operad including the ones which use configurations of points in the complex plane $\mathbb{C}$.

1.2. Operads of Feynman graphs. Kontsevich formality map $F$ is given by a sum $[\mathrm{Ko} 2$,

$$
F=\sum_{\Gamma \in \mathfrak{G}} c_{\Gamma} \Phi_{\Gamma}
$$

where the summation runs over a family of graphs $\mathfrak{G}$ and, for each graph $\Gamma \in \mathfrak{G} c_{\Gamma}$ is a complex number given by an integral over a fundamental chain in $\bar{C} \bullet \bullet \bullet(\mathbb{H})$ of a differential form $\Omega_{\Gamma}$, and $\Phi_{\Gamma}$ is a certain polydifferential operator. We show that the family $\mathfrak{G}$ can be equipped with a natural structure of a 2-coloured operad of Feynman graphs which admits a canonical representation

$$
\begin{array}{cccc}
\rho: & \mathfrak{G} & \longrightarrow & \mathcal{E} n d_{\left\{\mathcal{T}_{\text {poly }}(V), \mathcal{O}_{V}\right\}} \\
\Gamma & \longrightarrow & \Phi_{\Gamma}
\end{array}
$$

into the two-coloured endomorphism operad generated by the vector space of smooth (formal) polyvector fields $\mathcal{T}_{\text {poly }}(V)$ and the vector space, $\mathcal{O}_{V}$, of smooth (formal) functions on an affine space $V$. This representation is given precisely by the aforementioned polydifferential operators $\Phi_{\Gamma}$.

One can construct natural analogues of $\mathfrak{G}$ for any (coloured) operad of compactified configuration spaces, $\bar{C}$, studied in this paper. To distinguish these (coloured) operads of Feynman graphs from each other we use an appropriate subscript, $\mathfrak{G}_{\bar{C}}$, to indicate which geometric operad $\bar{C}$ an operad of Feynman diagrams $\mathfrak{G}$ is associated to (or, speaking plainly, which space the graphs from $\mathfrak{G}$ are drawn on).

1.3. De Rham field theories on $\bar{C}$. The numbers $c_{\Gamma}=\int_{\bar{C}, \bullet(\mathbb{H})} \Omega_{\Gamma}$ in the Kontsevich formula also have a clear operadic meaning. To explain it we have to articulate a new concept (cf. [Ko3]).

For any operad, $\bar{C}=\{\bar{C}(n)\}$, in the category of smooth manifolds with corners, the associated $\mathbb{S}$-module of de Rham algebras, $\Omega_{\bar{C}}=\left\{\Omega_{\bar{C}(n)}, d_{D R}\right\}$, is a dg cooperad (if equipped with a completed tensor product, see $₫ 8$ for details). Let $\mathfrak{G}_{\bar{C}}^{*}$ be the dual cooperad of Feynman graphs, and let $\check{\mathfrak{G}}_{\bar{C}} \subset \mathfrak{G}_{\bar{C}}^{*}$ be its sub-cooperad spanned by finite 
linear combinations of graphs. A de Rham field theory on $\bar{C}$ is, by definition, a morphism of dg cooperads,

$$
\begin{array}{ccc}
\Omega:\left(\check{\mathfrak{G}}_{\bar{C}}, 0\right) & \longrightarrow & \left(\Omega_{\bar{C}}, d_{D R}\right) \\
\Gamma & \longrightarrow & \Omega_{\Gamma}
\end{array}
$$

where $\check{\mathfrak{G}}_{\bar{C}}$ is equipped with the trivial differential (there exist variants of this definition in which $\check{\mathfrak{G}}_{\bar{C}}$ has a non-trivial differential but we do not need such variants in this paper). Any such a theory defines an associated morphism of dg operads,

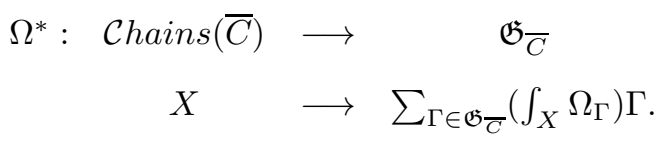

Therefore, any representation,

$$
\rho: \mathfrak{G}_{\bar{C}} \longrightarrow \mathcal{E} n d_{W}
$$

of the (coloured) operad of Feynman graphs in a (collection of) vector space(s) $W$ can be quantized as follows

$$
\rho^{\text {quant }}: \mathcal{F C h a i n s}(\bar{C}) \hookrightarrow \mathcal{C} \text { hains }(\bar{C}) \stackrel{\Omega^{*}}{\longrightarrow} \mathfrak{G}_{\bar{C}} \stackrel{\rho}{\longrightarrow} \mathcal{E} n d_{W} .
$$

When one applies this general construction to Kontsevich's configuration spaces, $\bar{C}=\bar{C}(\mathbb{H})$, and uses his formulae for $\Omega_{\Gamma}$ in terms of a propagator, then one obtains precisely his formality map as the quantization of the aforementioned standard representation $\mathfrak{G} \rightarrow \mathcal{E} n d_{\left\{\mathcal{T}_{\text {poly }}(V), \mathcal{O}_{V}\right\}}$. Note that Kontsevich formulae admit a natural extension from the suboperad of fundamental chains, $\mathcal{F C h a i n s}(\bar{C})$ to the full operad of chains in $\bar{C}(\mathbb{H})$; this extension plays no role in our paper but we refer to a beautiful work of Johan Alm A who employed this observation to construct another less obvious sub-operad of $\mathcal{C}$ hains $(\bar{C}(\mathbb{H}))$ and then used this new suboperad to extend explicitly Duflo-Kontsevich algebra isomorphism CaRo, Du, Ko2, MT, $\mathrm{PT}$

$$
H^{\bullet}(\mathfrak{g}, \odot \bullet \mathfrak{g}) \longrightarrow H^{\bullet}(\mathfrak{g}, U(\mathfrak{g})),
$$

at the level of cohomologies to an $\mathcal{A}_{\infty}$ quasi-isomorphism between the associated Chevalley-Eilenberg complexes equipped with certain $A_{\infty}$-structures. Here $\mathfrak{g}$ stands for an arbitrary finite-dimensional graded Lie algebra, and $U(\mathfrak{g})$ for its universal enveloping algebra.

Another useful output of this interpretation of Kontsevich's deformation quantization is that one can apply this technique to any operad of compactified configuration spaces and to any representation of the operad of Feynman graphs, not necessarily to the standard representation in $\mathcal{E} n d_{\left\{\mathcal{T}_{\text {poly }}(V), \mathcal{O}_{V}\right\}}$. We show several new explicit examples below.

1.4. Content of the paper. Section 2 reminds a well-known interpretation of Stasheff's associahedra (or, in essence, of the operad of $\mathcal{A}_{\infty}$-algebras) as compactified configuration spaces of points on the real line $\mathbb{R}$. In $\S 3$ we give a similar description of Stasheff's multiplihedra (or the 2-coloured operad, $\operatorname{Mor}\left(\mathcal{A}_{\infty}\right)$, of $\mathcal{A}_{\infty}$-morphisms of $\mathcal{A}_{\infty}$-algebras). The main novelty here is a new compactification of configuration spaces of points on $\mathbb{R}$ whose boundary strata involve not only collapsing points but also points going far away from each other in the standard Euclidean metric on $\mathbb{R}$; this construction is a 1-dimensional version of the 2-dimensional geometric model [Me2] for the 2-coloured operad $\operatorname{Mor}\left(\mathcal{L}_{\infty}\right)$. In fact we give in $₫ 3$ two inequivalent configuration space models for $\operatorname{Mor}\left(\mathcal{A}_{\infty}\right)$ and discuss at length their similarities and differences as the same idea will be repeated several times later in higher dimensions.

In 4 we remind Kontsevich's compactification [Ko2 of configuration spaces of points on the closed upper half-plane [Ko2] and the associated notion of open-closed homotopy algebra [KaSt]. In $\$ 5$ we discuss several configuration space models for the 2-coloured operad, $\operatorname{Mor}\left(\mathcal{L}_{\infty}\right)$, of $\mathcal{L}_{\infty}$ morphisms; one of them was studied earlier in Me2]. In $\S \sqrt{6}$ we construct two configuration space models, $\widehat{\mathfrak{C}}_{\bullet}, \bullet(\mathbb{H})$, for the operad, $\mathcal{M o r}\left(\mathcal{O C}_{\infty}\right)$, of morphisms of open-closed homotopy algebras.

Operads of Feynman graphs and their representations are studied in $\$ 7$ Re Rham field theories on operads of configuration spaces are introduced in $8 \sqrt[8]{8}$ a de Rham field theory on the Fulton-MacPherson compactification, $\bar{C}\left(\mathbb{R}^{d}\right)$, of points in $\mathbb{R}^{d}$ - one of the simplest in the class - is studied there in full details.

In 9 we consider several concrete quantized representations of operads of Feynman diagrams including the one which gives a strange non-flat $\mathcal{A}_{\infty}$-algebra structure on $\mathcal{T}_{\text {poly }}(V)$ induced from the standard homogeneous volume 
form on the circle $S^{1}$. We also consider a version of the Kontsevich construction in the 3-dimensional hyperbolic space and use it to give explicit formulae for a 1-parameter (homotopy trivial) deformation of the standard Gerstenhaber algebra structure in $\mathcal{T}_{\text {poly }}(V)$ which involves an infinite sequence of Bernoulli numbers.

In Sect. 10 we discuss de Rham field theories on configuration space models for the 2-coloured operad $\mathcal{M o r}\left(\mathcal{L}_{\infty}\right)$ and on the 4-coloured operad, $\widehat{\mathfrak{C}}_{\bullet}, \bullet(\mathbb{H})$. This machinery is expected to produce morphisms of open-closed homotopy algebras out of a propagator, $\omega$, on the following 3-dimensional version of the Kontsevich eye,

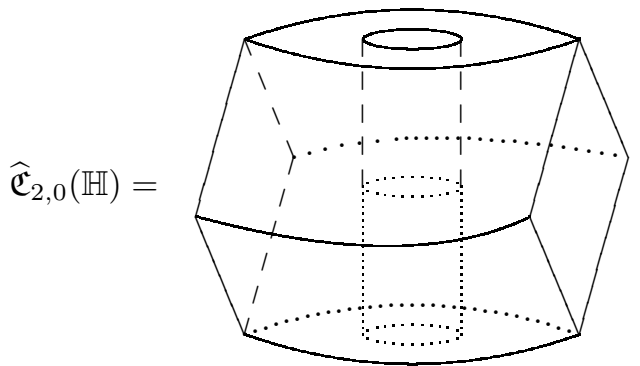

and give us explicit formulae for the homotopy action of the Grothendieck-Teichmueller group on deformation quantizations, an open problem which we hope to address elsewhere and which was the main motivation for writing this paper.

We assume that the reader knows the language of (coloured) operads. However in the Appendix we collected all the information about this concept which is necessary to read our text. We paid special attention to the presence and absence of units in operads as several operads of configuration spaces have no units so that some classical definitions of operads become inequivalent to each other.

We tried not to be sketchy and attempted to show every important detail of all the constructions and illustrate with examples every non-evident definition. Hence the size of this text.

1.5. Some notation. The set $\{1,2, \ldots, n\}$ is abbreviated to $[n]$; its group of automorphisms is denoted by $\mathbb{S}_{n}$. The cardinality of a finite set $A$ is denoted by \#A. If $V=\oplus_{i \in \mathbb{Z}} V^{i}$ is a graded vector space, then $V[k]$ stands for the graded vector space with $V[k]^{i}:=V^{i+k}$ and and $s^{k}$ for the associated isomorphism $V \rightarrow V[k]$; for $v \in V^{i}$ we set $|v|:=i$. For a pair of graded vector spaces $V_{1}$ and $V_{2}$, the symbol $\operatorname{Hom}_{i}\left(V_{1}, V_{2}\right)$ stands for the space of homogeneous linear maps of degree $i$, and $\operatorname{Hom}\left(V_{1}, V_{2}\right):=\bigoplus_{i \in \mathbb{Z}} \operatorname{Hom}_{i}\left(V_{1}, V_{2}\right)$; for example, $s^{k} \in \operatorname{Hom}_{-k}(V, V[k])$. If $\omega_{1}$ and $\omega_{2}$ are differential forms on manifolds $M_{1}$ and, respectively, $M_{2}$, then the form $p_{1}^{*}\left(\omega_{1}\right) \wedge p_{2}^{*}\left(\omega_{2}\right)$ on $M_{1} \times M_{2}$, where $p_{1}: M_{1} \times M_{2} \rightarrow M_{1}$ and $p_{2}: M_{1} \times M_{2} \rightarrow M_{2}$ are natural projections, is often abbreviated to $\omega_{1} \wedge \omega_{2}$.

We work throughout in the category of smooth manifolds with corners. However, all the main theorems of this paper hold true in the category of semialgebraic manifolds introduced in [KS] and further developed in [HLTV] so that in applications one can employ not only ordinary smooth differential forms but also $P A$-forms, where $P A$ stands for "piecewise semi-algebraic" as defined in the above mentioned papers. We use this freedom to change the category of geometric species we work in throughout the text.

\section{Associahedra as compactified configuration spaces of points on the real line}

2.1. Stasheff's associahedra and configuration spaces. Here we remind a well-known construction [St, Ko2] identifying the operad of $\mathcal{A}_{\infty}$-algebras with the fundamental chain complex 1 of the topological operad, $\bar{C}(\mathbb{R})=$ $\left\{\bar{C}_{n}(\mathbb{R})\right\}_{n \geq 2}$, of compactified configuration spaces of (equivalence classes of) points on the real line $\mathbb{R}$. Let

$$
\operatorname{Conf}_{n}(\mathbb{R}):=\{[n] \hookrightarrow \mathbb{R}\},
$$

be the space of all possible injections of the set $[n]$ into the real line $\mathbb{R}$. This space is a disjoint union of $n$ ! connected components each of which is isomorphic to the space

$$
\operatorname{Conf}_{n}^{o}(\mathbb{R})=\left\{x_{1}<x_{2}<\ldots<x_{n}\right\} .
$$

\footnotetext{
${ }^{1}$ All operads $\mathcal{C}=\left\{\mathcal{C}_{n}\right\}_{n \geq 1}$ of compactified configuration spaces considered in this paper are free as operads in the category of sets; the topological closures of its generators are called faces or fundamental chains of $\mathcal{C}$; moreover, the subspace of the chain operad of the topological operad $\mathcal{C}$ generated by the fundamental chains is always a $\operatorname{dg}$ suboperad called the fundamental chain operad of $\mathcal{C}$, or its face complex.
} 
The set $\operatorname{Conf}_{n}(\mathbb{R})$ has a natural structure of an oriented $n$-dimensional manifold with orientation on $\operatorname{Conf}{ }_{n}^{0}(\mathbb{R})$ given by the volume form $d x_{1} \wedge d x_{2} \wedge \ldots \wedge d x_{n}$; orientations of all other connected components are then fixed once we assume that the natural smooth action of $\mathbb{S}_{n}$ on $\operatorname{Conf}_{n}(\mathbb{R})$ is orientation preserving. In fact, we can (and often do) label points by an arbitrary finite set $I$, that is, consider the space of injections of sets,

$$
\operatorname{Conf}_{I}(\mathbb{R}):=\{I \hookrightarrow \mathbb{R}\} .
$$

A 2-dimensional Lie group $G_{(2)}=\mathbb{R}^{+} \ltimes \mathbb{R}$ acts freely on $\operatorname{Conf}_{n}(\mathbb{R})$ by the law,

$$
\begin{array}{cccc}
\operatorname{Conf}_{n}(\mathbb{R}) & \times \mathbb{R}^{+} \ltimes \mathbb{R} & \longrightarrow & \operatorname{Conf}_{n}(\mathbb{R}) \\
p=\left\{x_{1}, \ldots, x_{n}\right\} & (\lambda, \nu) & \longrightarrow & \lambda p+\nu:=\left\{\lambda x_{1}+\nu, \ldots, \lambda x_{n}+\nu\right\} .
\end{array}
$$

The action is free so that the quotient space,

$$
C_{n}(\mathbb{R}):=\operatorname{Conf}_{n}(\mathbb{R}) / G_{(2)}, \quad n \geq 2,
$$

is naturally an $(n-2)$-dimensional real oriented manifold equipped with a smooth orientation preserving action of the group $\mathbb{S}_{n}$. In fact,

$$
C_{n}(\mathbb{R})=C_{n}^{o}(\mathbb{R}) \times \mathbb{S}_{n}
$$

with orientation, $\Omega_{n}$, defined on $C_{n}^{o}(\mathbb{R}):=\operatorname{Conf}_{n}^{o}(\mathbb{R}) / G_{(2)}$ as follows: identify $C_{n}^{o}(\mathbb{R})$ with the subspace of $\operatorname{Conf}_{n}^{o}(\mathbb{R})$ consisting of points $\left\{0=x_{1}<x_{2}<\ldots<x_{n}=1\right\}$ and then set $\Omega_{n}:=d x_{2} \wedge \ldots \wedge d x_{n-1}$.

The space $C_{2}(\mathbb{R})$ is closed as it is the disjoint union, $C_{2}(\mathbb{R}) \simeq \mathbb{S}_{2}$, of two points. The topological compactification, $\bar{C}_{n}(\mathbb{R})$, of $C_{n}(\mathbb{R})$ for higher $n$ can be defined as $\bar{C}_{n}^{o}(\mathbb{R}) \times \mathbb{S}_{n}$ where $\bar{C}_{n}^{o}(\mathbb{R})$ is, by definition, the closure of an embedding,

$$
\begin{array}{ccc}
C_{n}^{o}(\mathbb{R}) & \longrightarrow & \left(\mathbb{R}^{2}\right)^{n(n-1)(n-2)} \\
\left(x_{i_{1}}, \ldots, x_{i_{n}}\right) & \longrightarrow & \prod_{i_{p} \neq i_{q} \neq i_{r} \neq i_{p}}\left[\left|x_{i_{p}}-x_{i_{q}}\right|:\left|x_{i_{q}}-x_{i_{r}}\right|:\left|x_{i_{p}}-x_{i_{r}}\right|\right] .
\end{array}
$$

Its codimension one strata are given by

$$
\partial \bar{C}_{n}^{o}(\mathbb{R})=\bigsqcup_{A} \bar{C}_{n-\# A+1}^{o}(\mathbb{R}) \times \bar{C}_{\# A}^{o}(\mathbb{R}),
$$

where the union runs over connected proper subsets, $A$, of the set $[1,2, \ldots, n]$ with $\# A \geq 2$. The fundamental chain operad of $\bar{C}(\mathbb{R})$ is a dg free operad (in the category of linear spaces) generated by the $\mathbb{S}$-module,

$$
\mathbb{K}\left[\mathbb{S}_{n}\right]=\langle\underset{\sigma(1) \sigma(2)}{\downarrow}\rangle_{\sigma(n)}, \quad n \geq 2,
$$

with the differential given by 2

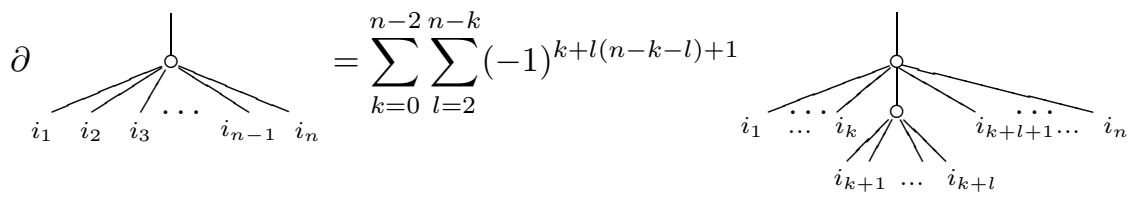

Therefore, the operad of fundamental chains of $\bar{C}(\mathbb{R})$ is nothing but the minimal resolution, $\mathcal{A} s s_{\infty}$, of the operad of associative algebras.

2.1.1. Example. $C_{3}^{0}(\mathbb{R})$ is an open interval,

$$
C_{3}^{0}(\mathbb{R})=(0,1) \simeq \stackrel{x_{1}=0}{\stackrel{x_{3}=1}{*}:-}
$$

Its compactification $\bar{C}_{3}^{0}(\mathbb{R})$ is, by definition, the closure of the following embedding,

$$
\left.i: \begin{array}{ccc}
C_{3}^{0}(\mathbb{R}) & \longrightarrow & \mathbb{R P}^{2} \\
& \left(x_{1}<x_{2}<x_{3}\right) & \longrightarrow
\end{array}\left[\left|x_{1}-x_{2}\right|:\left|x_{2}-x_{3}\right|: \mid x_{1}-x_{3}\right]\right]
$$

so that

$$
\bar{C}_{3}^{0}(\mathbb{R})=\overline{i\left(C_{3}^{0}(\mathbb{R})\right)}=i\left(C_{3}^{0}(\mathbb{R})\right) \sqcup[0: 1: 1] \sqcup[1: 0: 1]=(0,1) \sqcup(0) \sqcup(1)=[0,1]
$$

\footnotetext{
${ }^{2}$ This formula follows immediately from the above formula for the above formula for $\partial \bar{C}_{n}(\mathbb{R})$ except for the sign factor which compares the induced orientation on the boundary with the product orientation on the right hand side. We shall prove this sign factor in 2.3 below.
} 
Therefore,

$$
\partial \bigwedge_{12_{3}}=-\AA_{2}^{\alpha_{3}}+\underset{1}{q_{2}} .
$$

where

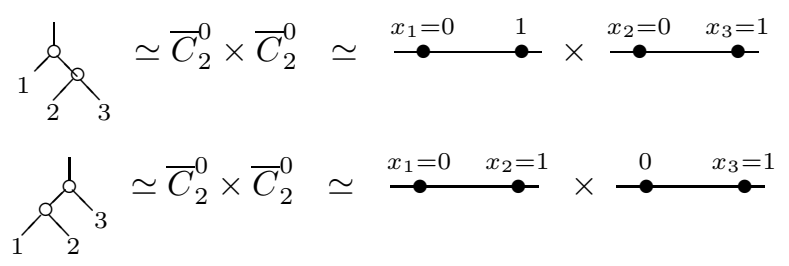

2.2. Smooth structure on $\bar{C}_{n}(\mathbb{R})$. The codimension $l$ boundary strata of $\bar{C}_{n}(\mathbb{R})$ is a disjoint union,

$$
\coprod_{T \in \mathcal{T}_{n, l}} \underbrace{\prod_{v \in V(T)} C_{\# \operatorname{In}(v)}(\mathbb{R})}_{C_{T}(\mathbb{R})}
$$

running over the set, $\mathcal{T}_{n, l}$, of all possible trees (built from the above corollas) with $l+1$ vertices and $n$ input legs which are labeled by elements of $[n]$. Here $V(T)$ stands for the set of vertices of a tree $T$ and $\operatorname{In}(v)$ for the set of input legs of a vertex $v$ (we also use below the symbol $E(T)$ to denote the set of internal edges of $T$ ). The resulting stratification,

$$
\bar{C}_{n}(\mathbb{R})=\coprod_{l \geq 0} \coprod_{T \in \mathcal{T}_{n, l}} C_{T}(\mathbb{R})
$$

can be used to make the compactified configuration space $\bar{C}_{n}(\mathbb{R})$ into a smooth manifold with corners. For that purpose we need to construct a coordinate chart $\mathcal{U}_{T}$ near the boundary stratum $C_{T}(\mathbb{R}) \subset \bar{C}_{n}(\mathbb{R})$ corresponding to an arbitrary tree $T$, say to this one

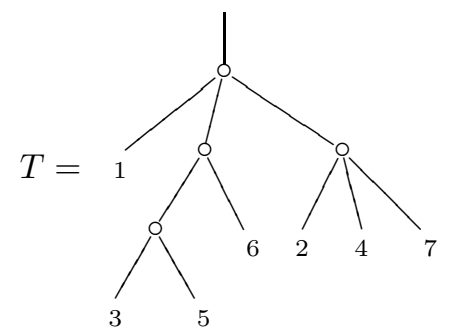

and then check that the gluing mappings at the intersections, $\mathcal{U}_{T} \cap \mathcal{U}_{T^{\prime}}$, of such charts are smooth. The construction of $\mathcal{U}_{T}$ goes in four steps (cf. Sect. 5.2 in [Ko2] which we discuss in some detail as it applies to all configuration spaces we study in this paper:

(i) Associate to $T$ a metric graph, $T_{\text {metric }}$, by assigning a small non-negative parameter $\varepsilon$ to each internal edge of $T$, e.g.

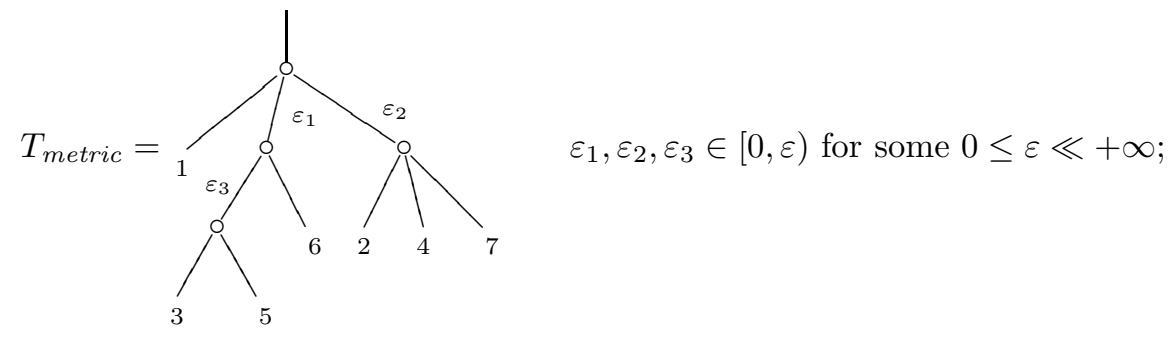

(ii) choose an $\mathbb{S}_{n}$-equivariant section, $\tau: C_{n}(\mathbb{R}) \rightarrow \operatorname{Conf}_{n}(\mathbb{R})$, of the natural projection $\operatorname{Conf}_{n}(\mathbb{R}) \rightarrow C_{n}(\mathbb{R})$ as well as an arbitrary smooth structure on its image, $C_{n}^{s t}(\mathbb{R}):=\tau\left(C_{n}(\mathbb{R})\right)$, which is often called the space of configurations in the standard position; for example, $C_{n}^{\text {st }}(\mathbb{R})$ can be chosen to be a subspace of Conf $_{n}(\mathbb{R})$ satisfying either the conditions $\sum_{i=1}^{n} x_{i}=0$ and $\sum_{i}\left|x_{i}\right|^{2}=1$, or the conditions that the leftmost point in the configuration is at 0 and the rightmost point is at 1 ; 
(iii) the required coordinate chart $\mathcal{U}_{T} \subset \bar{C}_{n}(\mathbb{R})$ is, by definition, isomorphic to the manifold with corners $[0, \varepsilon)^{\# E(T)} \times \prod_{v \in V(T)} C_{\# \operatorname{In}(v)}(\mathbb{R})$ and the isomorphism is given by a map,

$$
\alpha_{T}:[0, \varepsilon)^{\# E(T)} \times \prod_{v \in V(T)} C_{\# I n(v)}^{s t}(\mathbb{R}) \longrightarrow \mathcal{U}_{T}
$$

which one reads from the graph $T_{\text {metric }}$ by interpreting it as a substitution scheme of $\varepsilon$-magnified standard configurations; for example, the map $\alpha_{T}$ corresponding to the tree (3) is given by a continuous map,

$$
\begin{aligned}
& (0, \varepsilon)^{3} \times C_{3}^{s t}(\mathbb{R}) \times C_{2}^{s t}(\mathbb{R}) \times C_{3}^{s t}(\mathbb{R}) \times C_{2}^{s t}(\mathbb{R}) \quad \longrightarrow \quad C_{7}(\mathbb{R}) \\
& \left(\varepsilon_{1}, \varepsilon_{2}, \varepsilon_{3}\right) \times\left(x_{1}, x^{\prime}, x^{\prime \prime}\right) \times\left(x^{\prime \prime \prime}, x_{6}\right) \times\left(x_{2}, x_{4}, x_{7}\right) \times\left(x_{3}, x_{7}\right) \longrightarrow\left(y_{1}, y_{3}, y_{5}, y_{6}, y_{2}, y_{4}, y_{7}\right) \\
& y_{1}=x_{1} \quad y_{2}=\varepsilon_{2} x_{2}+x^{\prime \prime} \\
& y_{3}=\varepsilon_{1}\left(\varepsilon_{3} x_{3}+x^{\prime \prime \prime}\right)+x^{\prime} \quad y_{4}=\varepsilon_{2} x_{4}+x^{\prime \prime} \\
& y_{5}=\varepsilon_{1}\left(\varepsilon_{3} x_{5}+x^{\prime \prime \prime}\right)+x^{\prime} \quad y_{7}=\varepsilon_{2} x_{7}+x^{\prime \prime} \\
& y_{6}=\varepsilon_{1} x_{6}+x^{\prime},
\end{aligned}
$$

whose domain is formally extended to $[0, \varepsilon)^{3} \times C_{3}^{s t}(\mathbb{R}) \times C_{2}^{s t}(\mathbb{R}) \times C_{3}^{s t}(\mathbb{R}) \times C_{2}^{s t}(\mathbb{R})$; the boundary stratum $C_{T}(\mathbb{R})$ is given in $\mathcal{U}_{T}$ by the equations $\varepsilon_{1}=\varepsilon_{2}=\varepsilon_{3}=0$.

It is easy to check that the gluing mappings at every non-empty intersection $\mathcal{U}_{T} \cap \mathcal{U}_{T^{\prime}}$ are smooth (cf. Ga]).

We have $\bar{C}_{n}(\mathbb{R})=\mathcal{A}_{n} \times \mathbb{S}_{n}$, where $\mathcal{A}_{n}:=\bar{C}_{n}^{o}(\mathbb{R})$ is the $n$-th Stasheff's associahedron [St], for example

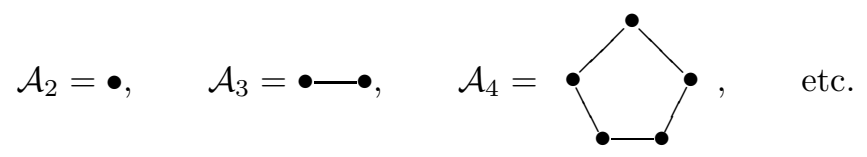

2.3. Induced orientation on the boundary strata. Let us prove the formula for signs in (1D), that is, let us compare the orientation, $\Omega_{T}$, induced on a generic codimension 1 boundary stratum $C_{T}(\mathbb{R}) \simeq \bar{C}_{n-l+1}(\mathbb{R}) \times \bar{C}_{l}(\mathbb{R}) \subset$ $\bar{C}_{n}(\mathbb{R})$ corresponding to a tree,

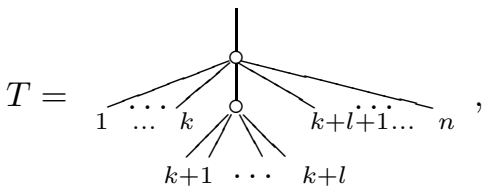

from the standard orientation, $\Omega$, on $\bar{C}_{n}(\mathbb{R})$ with the product, $\Omega_{1} \times \Omega_{2}$, of the standard orientations on $\bar{C}_{n-l+1}(\mathbb{R})$ and $\bar{C}_{l}(\mathbb{R})$. The upper corolla in $T$ corresponds to the configuration space $C_{n-l+1}(\mathbb{R})$ with the volume form in the standard coordinates (in which the left most point is at 0 at the right most point at 1 ) given by

$$
\Omega_{1}=d x_{2} \wedge \ldots \wedge d x_{k} \wedge d x_{\bullet} \wedge d x_{k+l+1} \wedge \ldots \wedge d x_{n-1} .
$$

The lower corolla corresponds to $C_{l}(\mathbb{R})$ with the volume form given in the standard coordinates by

$$
\Omega_{2}=d \bar{x}_{k+2} \wedge \ldots \wedge d \bar{x}_{k+l-1} .
$$

The inclusion $C_{T}(\mathbb{R}) \hookrightarrow \bar{C}_{n}(\mathbb{R})$ is best described in the coordinate chart $\mathcal{U}_{T}$ corresponding to the metric tree,

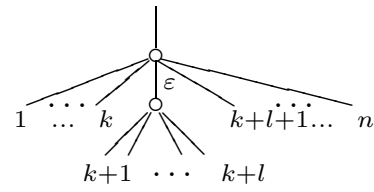

i.e. in the coordinates

$$
\left(\varepsilon, x_{2}, \ldots, x_{k}, x_{\bullet}, x_{k+l+1}, \ldots, x_{n-1}, \bar{x}_{2}, \bar{x}_{3} \ldots, \bar{x}_{k+l-1}\right) .
$$

These coordinates are related to the standard coordinates $0<y_{2}<\ldots<y_{n-1}<1$ on $C_{n}(\mathbb{R})$ as follows,

$$
\begin{gathered}
y_{2}=x_{2}, \quad y_{3}=x_{3}, \quad \ldots, \quad y_{k}=x_{k}, \\
y_{k+1}=x_{\bullet}, \quad y_{k+2}=\varepsilon \bar{x}_{k+2}+x_{\bullet}, \ldots, \quad y_{k+l-1}=\varepsilon \bar{x}_{k+l-1}+x_{\bullet}, \quad y_{k+l}=\varepsilon+x_{\bullet}, \\
y_{k+l+1}=x_{k+l+1}, \quad \ldots, \quad y_{n-1}=x_{n-1}
\end{gathered}
$$


so that the orientation form on $C_{n}(\mathbb{R})$ is given in the "metric tree" coordinates as follows,

$$
\begin{aligned}
\Omega & =d y_{2} \wedge d y_{3} \wedge \ldots \wedge d y_{n-1} \\
& =\varepsilon^{l-2} d x_{1} \wedge \ldots \wedge d x_{k} \wedge d x_{\bullet} \wedge d \bar{x}_{k+2} \wedge \ldots \wedge d \bar{x}_{k+l-1} \wedge d \varepsilon \wedge d x_{k+l+1} \wedge \ldots \wedge d x_{n-1} \\
& =(-1)^{k+l+1+(l-2)(n-k-l-1)} \varepsilon^{l-2} d \varepsilon \Omega_{1} \wedge \Omega_{2} \\
& =(-1)^{k+1+l(n-k-l)} \varepsilon^{l-2} d \varepsilon \Omega_{1} \wedge \Omega_{2} .
\end{aligned}
$$

As the boundary $C_{T}(\mathbb{R}) \hookrightarrow \bar{C}_{n}$ is given by the equation $\varepsilon=0$ and $\varepsilon \geq 0$, the induced orientation on $C_{T}(\mathbb{R})$ is given by the form

$$
\Omega_{T}=-(-1)^{k+1+l(n-k-l)} \Omega_{1} \wedge \Omega_{2}=(-1)^{k+l(n-k-l)} \Omega_{1} \wedge \Omega_{2}
$$

proving thereby the sign formula in (11).

\subsection{An equivalent definition of $\bar{C} \cdot(\mathbb{R})$. Let}

$$
\widetilde{\operatorname{Conf}_{n}}(\mathbb{R}):=\{[n] \rightarrow \mathbb{R}\}
$$

be the space of all possible (not necessarily injective) maps of the set $[n]$ into the real line $\mathbb{R}$. For a configuration $p=\left(x_{i_{1}}, \ldots x_{i_{n}}\right)$ in $\operatorname{Conf}_{n}(\mathbb{R})$ or in $\widetilde{\operatorname{Conf}}_{n}(\mathbb{R})$ we set

$$
x_{c}(p):=\frac{1}{n} \sum_{k=1}^{n} x_{i_{k}}, \quad\|p\|:=\sqrt{\sum_{k=1}^{n}\left|x_{i_{k}}-x_{c}(p)\right|^{2}} .
$$

Recall that each space $C_{n}(\mathbb{R})$ can be identified with a subspace,

$$
C_{n}^{s t}(\mathbb{R}):=\left\{p \in \operatorname{Conf}_{n}(\mathbb{R}) \mid x_{c}(p)=0, \quad\|p\|=1\right\} .
$$

Define next a compact space,

$$
\widetilde{C}_{n}^{s t}(\mathbb{R}):=\left\{p \in \widetilde{\operatorname{Conf}_{n}}(\mathbb{R}) \mid x_{c}(p)=0,\|p\|=1\right\}
$$

which contains $C_{n}^{s t}(\mathbb{R})$ as a subspace. For any subset $A \subseteq[n]$ there is a natural map

$$
\begin{aligned}
& \pi_{A}: \quad C_{n}(\mathbb{R}) \quad \longrightarrow \quad C_{A}(\mathbb{R}) \\
& p=\left\{x_{i}\right\}_{i \in[n]} \longrightarrow p_{A}:=\left\{x_{i}\right\}_{i \in A}
\end{aligned}
$$

which forgets all the points labeled by elements of the complement $[n] \backslash A$.

The compactification, $\bar{C}_{n}(\mathbb{R})$, can be defined as the closure of the embedding $\mathrm{AT}$,

$$
C_{n}(\mathbb{R}) \stackrel{\prod \pi_{A}}{\longrightarrow} \prod_{\substack{A \subseteq[n] \\ \# A \geq 2}} C_{A}(\mathbb{R}) \stackrel{\simeq}{\longrightarrow} \prod_{\substack{A \subseteq[n] \\ \# A \geq 2}} C_{A}^{s t}(\mathbb{R}) \hookrightarrow \prod_{\substack{A \subseteq[n] \\ \# A \geq 2}} \widetilde{C}_{A}^{s t}(\mathbb{R}) .
$$

For example, consider the case $n=3$,

$$
\begin{aligned}
& \begin{array}{lllllll}
i: & C_{3}(\mathbb{R}) & \widetilde{C}_{123}^{s t}(\mathbb{R}) & \times & \widetilde{C}_{12}^{s t}(\mathbb{R}) & \times & \widetilde{C}_{23}^{s t}(\mathbb{R})
\end{array} \\
& p=\left(x_{1}, x_{2}, x_{3}\right) \longrightarrow \frac{p-x_{c}(p)}{\| p||} \quad\left(\frac{x_{1}-x_{2}}{2\left|x_{2}-x_{1}\right|}, \frac{x_{2}-x_{1}}{2\left|x_{2}-x_{1}\right|}\right)=\left(-\frac{1}{2}, \frac{1}{2}\right) \quad\left(\frac{x_{2}-x_{3}}{2\left|x_{2}-x_{1}\right|}, \frac{x_{3}-x_{2}}{2\left|x_{2}-x_{3}\right|}\right)=\left(-\frac{1}{2}, \frac{1}{2}\right)
\end{aligned}
$$

It is clear that $\overline{i\left(C_{3}(\mathbb{R})\right)}$ is the union of $C_{3}(\mathbb{R})=(0,1)$ with two limiting points, 0 corresponding to $x_{1}-x_{2} \rightarrow 0$ and 1 corresponding to $x_{2}-x_{3} \rightarrow 0$, i.e. again $\bar{C}_{3}(\mathbb{R})=[0,1]$.

\section{Multiplihedra as compactified configuration spaces of points on the real line}

Here we construct two different compactifications of configuration spaces of distinct points on the real line both of which come equipped with the structure of a two coloured operad in the category of smooth manifolds with corners and share one and the same property: the associated face complex is precisely the 2-coloured operad, $\operatorname{Mor}\left(\mathcal{A}_{\infty}\right)$, whose representations are the same as a pair of $\mathcal{A}_{\infty}$-algebras together with an $\mathcal{A}_{\infty}$ morphism between them. These compactifications involve not only strata of collapsing points but also strata of points going far away from each other with respect to the standard Euclidean metric on $\mathbb{R}$. 
3.1. The first configuration space model. Let a 1-dimensional Lie group $G_{(1)}=\mathbb{R}$ act on the configuration space $\operatorname{Conf}_{n}(\mathbb{R})$ by translations,

$$
\begin{aligned}
& \operatorname{Conf}_{n}(\mathbb{R}) \times \mathbb{R} \quad \longrightarrow \quad \operatorname{Conf}_{n}(\mathbb{R}) \\
& \left(p=\left\{x_{1}, \ldots, x_{n}\right\}, \nu\right) \longrightarrow p+\nu:=\left\{x_{1}+\nu, \ldots, x_{n}+\nu\right\}
\end{aligned}
$$

This action is free so that the quotient space,

$$
\mathfrak{C}_{n}(\mathbb{R}):=\operatorname{Conf}_{n}(\mathbb{R}) / G_{(1)},
$$

is a naturally oriented $(n-1)$-dimensional real manifold equipped with a smooth action of the group $\mathbb{S}_{n}$ (one defines analogously $\mathfrak{C}_{A}(\mathbb{R}):=\operatorname{Conf}_{A}(\mathbb{R}) / G_{(1)}$ for any non-empty set $A$ ). The space $\mathfrak{C}_{1}(\mathbb{R})$ is a point and hence closed. For $n \geq 2$ we have an $\mathbb{S}_{n}$-equivariant isomorphism,

$$
\begin{aligned}
\Psi_{n}: \quad \begin{array}{c}
\mathfrak{C}_{n}(\mathbb{R}) \\
\quad p=\left(x_{i_{1}}, \ldots, x_{i_{n}}\right)
\end{array} & \longrightarrow\left(\frac{p-x_{c}(p)}{\| p},\|p\|\right)
\end{aligned}
$$

Note that the configuration $\frac{p-x_{c}(p)}{\|p\|}$ is $\mathbb{R}^{+} \ltimes \mathbb{R}$-invariant and hence represents a uniquely defined point in $C_{n}(\mathbb{R})$.

For any subset $A \subseteq[n]$ there is a natural map

$$
\begin{array}{ccc}
\pi_{A}: & \mathfrak{C}_{n}(\mathbb{R}) \\
p=\left\{x_{i}\right\}_{i \in[n]} & \longrightarrow & \mathfrak{C}_{A}(\mathbb{R}) \\
& \longrightarrow=\left\{x_{i}\right\}_{i \in A}
\end{array}
$$

which forgets all points labeled by elements of the complement $[n] \backslash A$ (which can be empty). We have $\mathfrak{C}_{n}(\mathbb{R})=$ $\mathfrak{C}_{n}^{0}(\mathbb{R}) \times \mathbb{S}_{n}$ where $\mathfrak{C}_{n}^{0}(\mathbb{R}):=\operatorname{Conf}_{n}^{o}(\mathbb{R}) / G_{(1)}$.

A topological compactification, $\widehat{\mathfrak{C}}_{n}(\mathbb{R})$, of $\mathfrak{C}_{n}(\mathbb{R})$ can be defined as $\widehat{\mathfrak{C}}_{n}^{o}(\mathbb{R}) \times \mathbb{S}_{n}$ where $\widehat{\mathfrak{C}}_{n}^{o}(\mathbb{R})$ is the closure of a composition (cf. [Me2]),

$$
\mathfrak{C}_{n}^{0}(\mathbb{R}) \stackrel{\prod \pi_{A}}{\longrightarrow} \prod_{A \subseteq[n]} \mathfrak{C}_{A}(\mathbb{R}) \stackrel{\prod_{A}}{\longrightarrow} \prod_{A \subseteq[n]} C_{A}^{s t}(\mathbb{R}) \times(0,+\infty) \hookrightarrow \prod_{A \subseteq[n]} \widetilde{C}_{A}^{s t}(\mathbb{R}) \times[0,+\infty] .
$$

here the product runs over connected nonempty subsets, $A$, of the set $[1,2, \ldots, n]$. Thus all the limiting points in this compactification come from configurations when a group or groups of points move too close to each other within each group (as in the case of $\bar{C}_{n}(\mathbb{R})$ ) and/or a group or groups of points are moving too far (with respect to the relative Euclidean distances inside each group) away from each other.

It is not hard to see from the above definition (we refer to 3 3.1.2 below for a detailed discussion of several explicit examples) that the codimension 1 boundary strata in $\widehat{\mathfrak{C}}_{n}(\mathbb{R})$ are given by

$$
\partial \widehat{\mathfrak{C}}_{n}(\mathbb{R})=\bigcup_{\substack{n=p+q+r \\ p, r \geq 0, q \geq 2}} \widehat{\mathfrak{C}}_{n-r+1}(\mathbb{R}) \times \bar{C}_{r}(\mathbb{R}) \bigcup_{\substack{n=n_{1}+\ldots+n_{k} \\ 2 \leq k \leq n \\ n_{1}, \ldots, n_{k} \geq 1}} \bar{C}_{k}(\mathbb{R}) \times \widehat{\mathfrak{C}}_{n_{1}}(\mathbb{R}) \times \ldots \times \widehat{\mathfrak{C}}_{n_{k}}(\mathbb{R})
$$

where

- the first summation runs over all possible partitions of the form

$$
\left\{x_{i_{1}}, \ldots, x_{i_{n}}\right\}=\left\{x_{i_{1}}, \ldots, x_{i_{p}}\right\} \sqcup\left\{x_{i_{p+1}}, \ldots, x_{i_{p+q}}\right\} \sqcup\left\{x_{i_{p+q+1}}, \ldots, x_{i_{n}}\right\}
$$

with the corresponding stratum $\mathfrak{C}_{n-r+1}(\mathbb{R}) \times C_{r}(\mathbb{R})$ describing limit configurations in which the points $\left\{x_{i_{p+1}}, \ldots, x_{i_{p+q}}\right\}$ collapse into a single point in the real line $\mathbb{R}$, and

- the second summation runs over all possible partitions of the form

$$
\left\{x_{i_{1}}, \ldots, x_{i_{n}}\right\}=\underbrace{\left\{x_{i_{1}}, \ldots, x_{i_{n_{1}}}\right\}}_{I_{1}} \sqcup \underbrace{\left\{x_{i_{n_{1}+1}}, \ldots, x_{i_{n_{1}+n_{2}}}\right\}}_{I_{2}} \sqcup \ldots \sqcup \underbrace{\left\{x_{i_{n_{1}+\ldots+n_{k-1}+1}}, \ldots, x_{i_{n}}\right\}}_{I_{k}}
$$

with the corresponding stratum $C_{k}(\mathbb{R}) \times \mathfrak{C}_{n_{1}}(\mathbb{R}) \times \ldots \times \mathfrak{C}_{n_{k}}(\mathbb{R})$ describing limit configurations in which the distances between different configurations $I_{i}$ and $I_{j}$ tend to $+\infty$ while the diameter of each such a configuration stands finite.

By analogy to $\overline{2.1}$ the collection of spaces $\left\{\bar{C} \cdot(\mathbb{R}) \sqcup \widehat{\mathfrak{C}}_{n}(\mathbb{R}) \sqcup \bar{C} \bullet(\mathbb{R})\right\}$ is naturally a dg topological operad, but this time a two coloured dg operad. Note that the faces of the type $C_{k}(\mathbb{R})$ appear in the natural stratification of $\widehat{\mathfrak{C}}_{n}(\mathbb{R})$ in two ways - as the strata of collapsing points and as the strata controlling groups of points at "infinity" — and 
they never intersect in $\widehat{\mathfrak{C}}_{n}(\mathbb{R})$. For that reason we have to assign to these two groups of faces different colours and represent collapsing $\bar{C} \cdot(\mathbb{R})$-strata by, say, solid white corollas

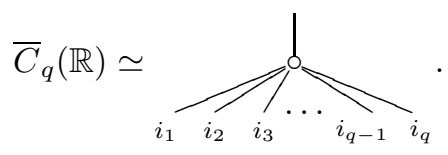

and $\bar{C} \cdot(\mathbb{R})$-stratum at "infinity" by, say, dashed white corollas

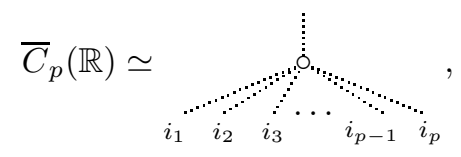

Finally, representing the faces $\widehat{\mathfrak{C}} \cdot(\mathbb{R})$ by the black corollas

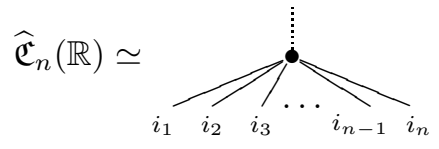

we can rewrite the boundary differential (11) in the associated face complex in a more informative way

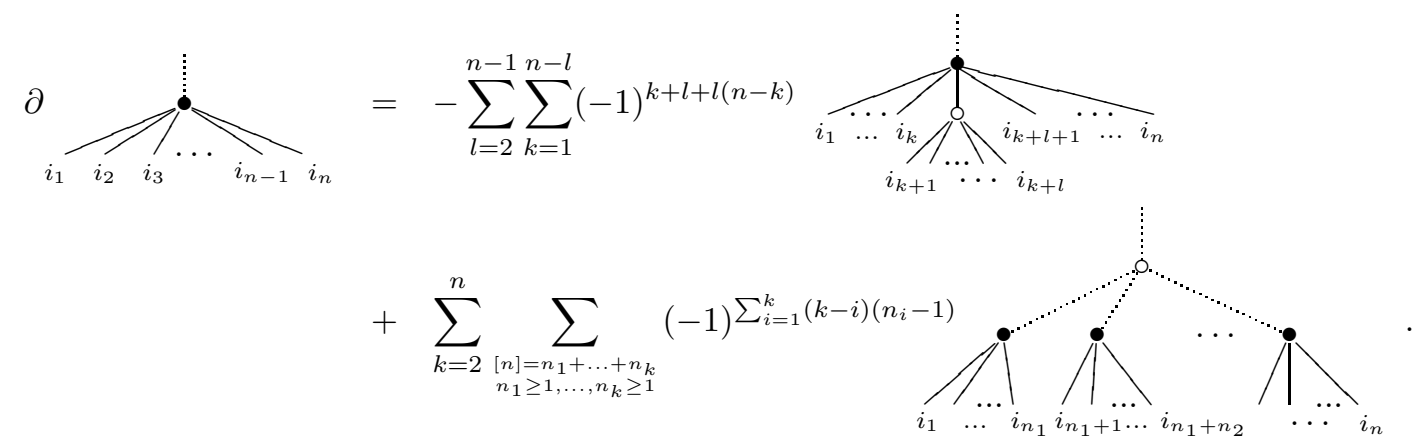

which takes into account signs coming from natural orientations of the faces. The next statement is now obvious.

3.1.1. Proposition. The face complex of the disjoint union $\bar{C} \bullet(\mathbb{R}) \sqcup \widehat{\mathfrak{C}}_{\bullet}(\mathbb{R}) \sqcup \bar{C} \bullet(\mathbb{R})$ has naturally a structure of a dg free non-unital 2-coloured operad of transformation type,

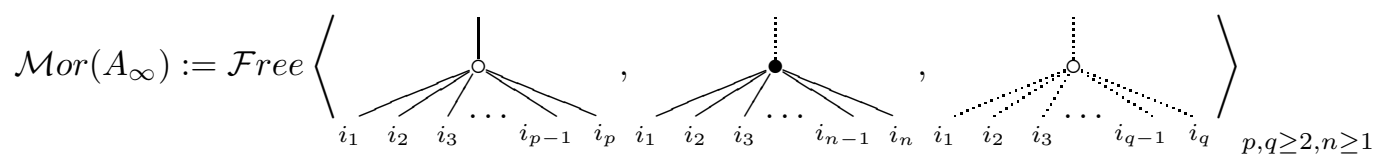

whose representations in a pair of vector spaces $V^{\text {in }}$ and $V^{\text {out }}$ are in 1-1 correspondence with with the triples, $\left(\mu_{\bullet}^{\text {in }}, \mu_{\bullet}^{\text {out }}, f_{\bullet}\right)$, consisting of an $\mathcal{A}_{\infty}$-structure $\mu_{\bullet}^{\text {in }}$ on the space $V^{\text {in }}$, an $\mathcal{A}_{\infty}$-structure $\mu_{\bullet}^{\text {out }}$ on the space $V^{\text {out }}$, and of an $\mathcal{A}_{\infty}$-morphisms, $f_{\bullet}:\left(V^{\text {in }}, \mu_{\bullet}^{\text {in }}\right) \rightarrow\left(V^{\text {out }}, \mu_{\bullet}^{\text {out }}\right)$, of $\mathcal{A}_{\infty}$-algebras

3.1.2. Examples. (i) $\mathfrak{C}_{1}(\mathbb{R})$ is already compact so that $\widehat{\mathfrak{C}}_{1}(\mathbb{R})=\mathfrak{C}_{1}(\mathbb{R})$.

(ii) $\mathfrak{C}_{2}(\mathbb{R})$ is isomorphic to $(0,+\infty)$. Its compactification, $\overline{\mathfrak{C}}_{2}(\mathbb{R})$ is given as the closure of the embedding,

$$
\begin{aligned}
& C_{2}(\mathbb{R}) \quad \longrightarrow \quad \widetilde{C}_{2}^{s t}(\mathbb{R}) \quad \times \quad[0,+\infty] \\
& \left\{x_{1}<x_{2}\right\} \quad \longrightarrow \quad\left(-\frac{1}{\sqrt{2}}, \frac{1}{\sqrt{2}}\right) \quad \frac{1}{\sqrt{2}}\left|x_{1}-x_{2}\right|,
\end{aligned}
$$

and hence is isomorphic to the closed interval $[0,+\infty]$. In terms of fundamental chains we get,

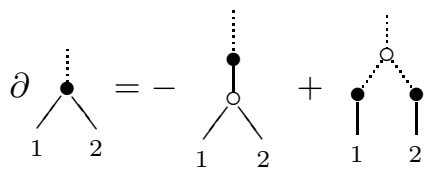

where the first term in the r.h.s. represents the limit configuration $\left|x_{1}-x_{2}\right| \rightarrow 0$ and the second one the limit configuration $\left|x_{1}-x_{2}\right| \rightarrow+\infty$. 
(iii) the compactification $\widehat{\mathfrak{C}}_{3}(\mathbb{R})$ is defined as the closure of an embedding 3 ,

$$
\begin{aligned}
& \begin{array}{lllll}
\mathfrak{C}_{3}(\mathbb{R}) \quad & \widetilde{C}_{3}^{s t}(\mathbb{R}) \times[0,+\infty] & \times & \widetilde{C}_{12}^{s t}(\mathbb{R}) \times[0,+\infty] \quad \times \quad \widetilde{C}_{23}^{s t}(\mathbb{R}) \times[0,+\infty]
\end{array} \\
& p=\left\{x_{1}<x_{2}<x_{3}\right\} \quad \longrightarrow \quad\left(\frac{p-x_{c}(p)}{\|p\|},\|p\|\right) \quad\left\|p_{12}\right\|=\frac{1}{\sqrt{2}}\left|x_{1}-x_{2}\right| \quad|| p_{23} \|=\frac{1}{\sqrt{2}}\left|x_{2}-x_{3}\right| .
\end{aligned}
$$

The codimension 1 boundary strata of $\widehat{\mathfrak{C}}_{3}(\mathbb{R})$ decomposes into strata determined by various limit values of the parameters $\|p\|,\left\|p_{12}\right\|$ and $\left\|p_{23}\right\|$ as follows:

(a) the stratum, $\bigcap_{1}^{\infty} \simeq \mathfrak{C}_{1}(\mathbb{R}) \times C_{3}^{s t}(\mathbb{R})$, is given by $\|p\|=\left\|p_{12}\right\|=\left\|p_{23}\right\|=0$; it represents the limit configurations in which all three points collapse into a single point in $\mathfrak{C}_{1}$ in such a way that the ratio $\frac{p-x_{c}(p)}{\|p\|}$ gives in the limit a well-defined point in $C_{3}^{s t}(\mathbb{R})$; any point in this boundary stratum can be obtained as the $\lambda \rightarrow 0$ limit of a configuration $\left(\lambda x_{1}, \lambda x_{2}, \lambda x_{3}\right) \in \mathfrak{C}_{3}(\mathbb{R})$ for some fixed $\left(x_{1}, x_{2}, x_{3}\right) \in C_{3}^{s t}(\mathbb{R})$;

(b) the stratum,

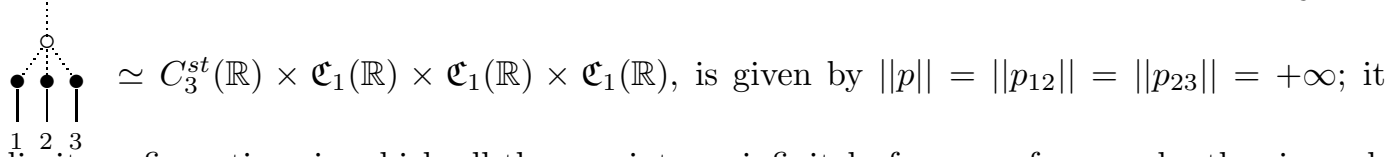
represents the limit configurations in which all three points go infinitely far away from each other in such a way that the ratio $\frac{p-x_{c}(p)}{\|p\|}$ gives in the limit a well-defined point in $C_{3}^{s t}(\mathbb{R})$; any point in this boundary stratum can be obtained as the $\lambda \rightarrow+\infty$ limit of a configuration $\left(\lambda x_{1}, \lambda x_{2}, \lambda x_{3}\right) \in \mathfrak{C}_{3}(\mathbb{R})$ for some fixed $\left(x_{1}, x_{2}, x_{3}\right) \in C_{3}^{s t}(\mathbb{R})$

(c) the stratum, represents the limit configurations in which the point 1 goes infinitely far away from the points 2 and 3 in such a way that the ratio $\frac{p-x_{c}(p)}{\|p\|}$ is well-defined; using translation freedom we can fix $x_{c}=0$ so that

$$
\frac{p}{\|p\|}=\frac{\left(x_{1}=:-\lambda, \frac{1}{2}\left(\lambda+\left(x_{2}-x_{3}\right)\right), \frac{1}{2}\left(\lambda+\left(x_{3}-x_{2}\right)\right)\right)}{\sqrt{\lambda^{2}+\frac{1}{4}\left(\lambda-\left(x_{3}-x_{2}\right)\right)^{2}+\frac{1}{4}\left(\lambda+\left(x_{3}-x_{2}\right)\right)^{2}}} \stackrel{\lambda \rightarrow+\infty}{\longrightarrow}\left(-\frac{\sqrt{2}}{\sqrt{3}}, \frac{1}{\sqrt{6}}, \frac{1}{\sqrt{6}}\right) .
$$

Thus in the limit the images of the points $x_{2}$ and $x_{3}$ in $\widetilde{C}_{3}(\mathbb{R})$ collapse into a single point. Any point in this boundary stratum can be obtained as the $\lambda \rightarrow+\infty$ limit of a configuration of the form $(-\lambda, \lambda+\Delta, \lambda-\Delta) \in$ $\mathfrak{C}_{3}(\mathbb{R})$ for some $\Delta \in \mathbb{R}$

(d) the stratum,<smiles>C1CCCCC1</smiles>

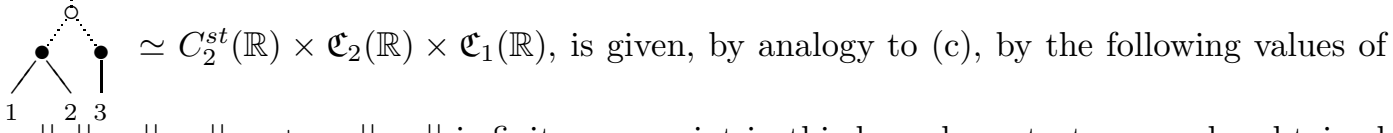
the parameters: $\|p\|=\left\|p_{23}\right\|=+\infty,\left\|p_{12}\right\|$ is finite; any point in this boundary stratum can be obtained as the $\lambda \rightarrow+\infty$ limit of a configuration of the form $(-\lambda-\Delta,-\lambda+\Delta, \lambda) \in \mathfrak{C}_{3}(\mathbb{R})$ for some $\Delta \in \mathbb{R}$;

(e) the stratum, $\overbrace{1} \simeq C_{2}^{s t}(\mathbb{R}) \times \mathfrak{C}_{1}(\mathbb{R}) \times \mathfrak{C}_{2}(\mathbb{R})$, is given by $0<\|p\|,\left\|p_{12}\right\|<+\infty,\left\|p_{23}\right\|=0$; it represents the limit configurations in which all the points are at a finite distance from each other and the points 2 and 3 collapse into a single point in $\mathfrak{C}_{2}(\mathbb{R})$; any point in this boundary stratum can be obtained as the $\lambda \rightarrow 0$ limit of a configuration of the form $(-x, x-\lambda, x+\lambda) \in \mathfrak{C}_{3}(\mathbb{R})$ for some $x \in \mathbb{R}$;

(f) the stratum,

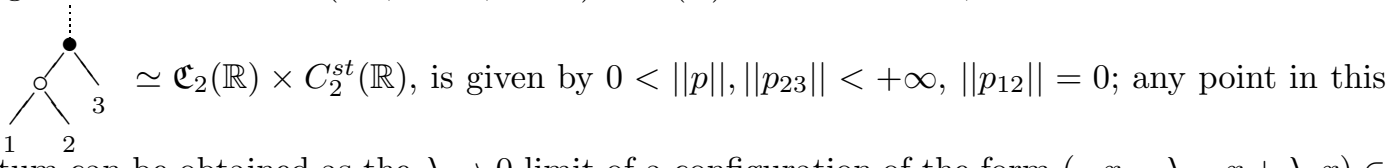
boundary stratum can be obtained as the $\lambda \rightarrow 0$ limit of a configuration of the form $(-x-\lambda,-x+\lambda, x) \in$ $\mathfrak{C}_{3}(\mathbb{R})$ for some $x \in \mathbb{R}$;

\footnotetext{
${ }^{3}$ It should be clear from the context that the subscript $12 \mathrm{in}$, say, $\widetilde{C}_{12}^{s t}(\mathbb{R})$ refers to a subset of [3] rather than to a natural number.
} 
Taking into account natural orientations we can finally summarize the above discussion in a single formula

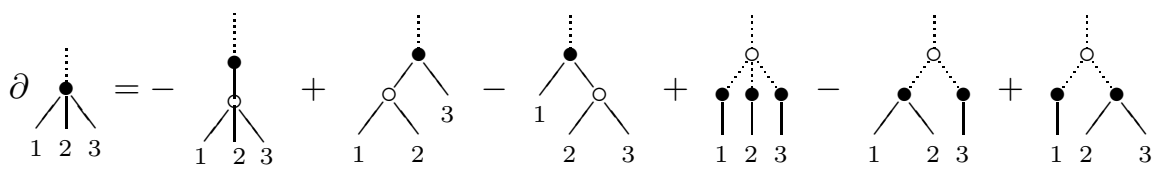

which is in agreement with (7).

A choice of a total ordering on the set $[n]$ (say, the natural one, $1<2<\ldots<n$ ), gives an equivariant smooth isomorphism $\overline{\mathfrak{C}}_{n}(\mathbb{R})=\mathcal{J}_{n} \times \mathbb{S}_{n}$, where $\mathcal{J}_{n}$ is the $n$-th Stasheff's multiplihedron [St], for example

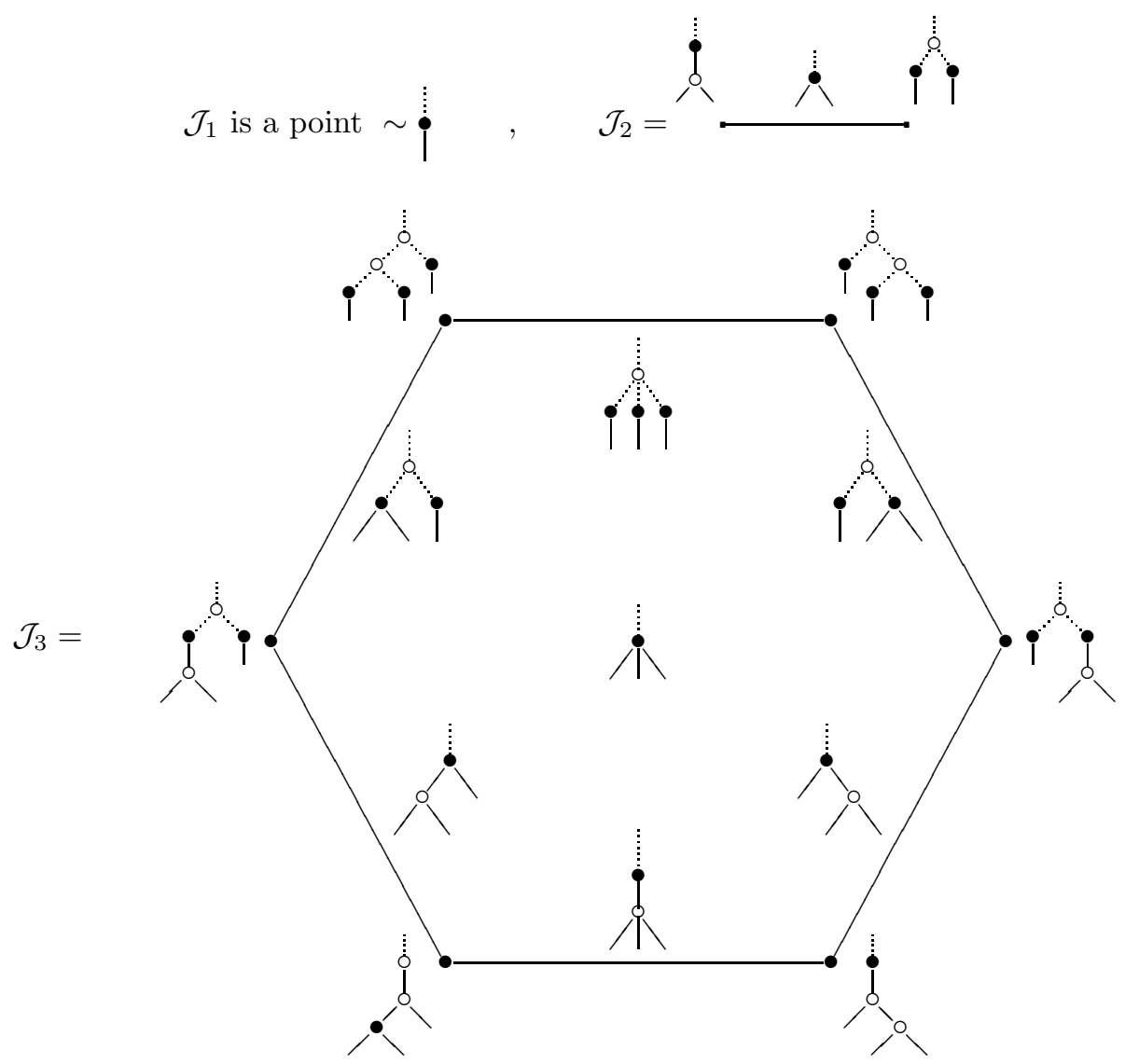

3.1.3. A smooth atlas on the compactification $\widehat{\mathfrak{C}}_{\bullet}(\mathbb{R})$. Using metric graphs we can make $\widehat{\mathfrak{C}}_{\bullet}(\mathbb{R})$ into a smooth manifold with corners by constructing a coordinate chart, $\mathcal{U}_{T}$, near the boundary stratum corresponding to an arbitrary tree $T \in \mathcal{M} \operatorname{or}\left(A_{\infty}\right)$ containing at least one black vertex as follows:

(i) Associate to $T$ a metric graph, $T_{\text {metric }}$, by assigning

(a) to every internal edge of $T$ of the form $\{$ a small positive real number $\varepsilon \ll+\infty$;

(b) to every (if any) white vertex of a dashed corolla in $T$ a large positive real number $\tau \gg 0$,

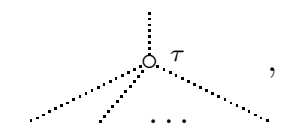

(c) to every (if any) two vertex subgraph of $T_{\text {metric }}$ of the form ${\stackrel{0}{\tau_{1}}}_{\tau_{2}}^{2}$ an inequality $\tau_{1} \gg \tau_{2} \gg 0$. This can be understood as a relation $\tau_{2}=\varepsilon_{12} \tau_{1}$ for some small parameter $\varepsilon_{12}$.

${ }^{4}$ If $T$ does not contain black vertices, then the associated boundary stratum lies in $\bar{C} \bullet(\mathbb{R})$ and the construction of $\mathcal{U}_{T}$ goes as in Subsection 2.1. 


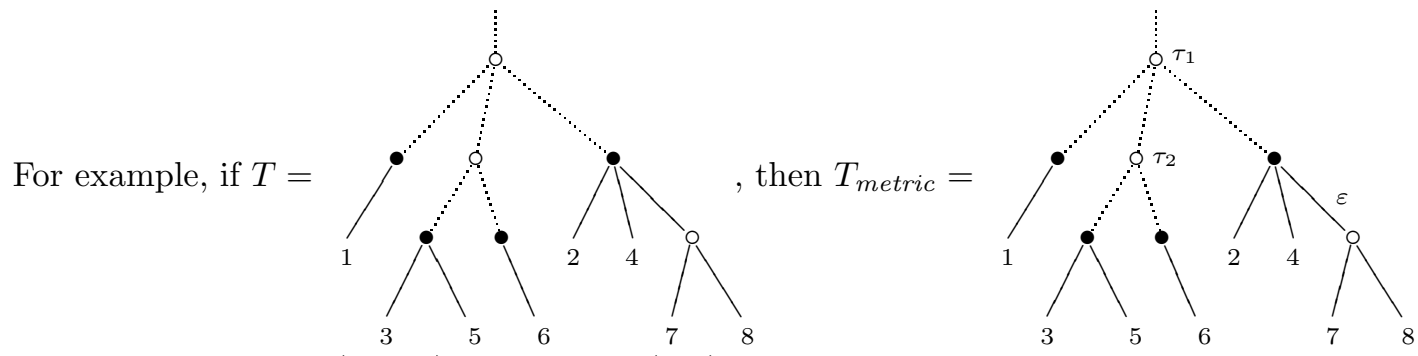

with $\tau_{1}, \tau_{2}=\varepsilon_{12} \tau_{1} \in(l,+\infty)$ and $\varepsilon_{12}, \varepsilon \in(0, s)$ for some real numbers $l \gg 0$ and $s \ll+\infty$.

(ii) Choose an equivariant section, $s: \mathfrak{C}_{n}(\mathbb{R}) \rightarrow \operatorname{Conf}_{n}(\mathbb{R})$, of the natural projection $\operatorname{Conf}_{n}(\mathbb{R}) \rightarrow \mathfrak{C}_{n}(\mathbb{R})$ as well as an arbitrary smooth structure on its image, $\mathfrak{C}_{n}^{s t}(\mathbb{R}):=s\left(\mathfrak{C}_{n}(\mathbb{R})\right)$, which is called the space of configurations in the standard position; for example, $\mathfrak{C}_{n}^{s t}(\mathbb{R})$ can be chosen to be the subspace of $\operatorname{Conf}_{n}(\mathbb{R})$ satisfying the condition $\sum_{i=1}^{n} x_{i}=0$; in particular, $\mathfrak{C}_{1}^{s t}(\mathbb{R})=0 \in \mathbb{R}$.

(iii) the required coordinate chart $\mathcal{U}_{T} \subset \overline{\mathfrak{C}}_{n}(\mathbb{R})$ is, by definition, isomorphic to the manifold with corners,

$$
(l,+\infty]^{\# V_{0}^{d}(T)} \times[0, s)^{\# E_{0}^{\bullet}(T)} \times \prod_{v \in V_{0}(T)} C_{\# \operatorname{In}(v)}^{s t}(\mathbb{R}) \times \prod_{v \in V_{\bullet}(T)} \mathfrak{C}_{\# \operatorname{In}(v)}^{s t}(\mathbb{R})
$$

where $V_{\circ}(T)$ is the set of white vertices of $T, V_{\circ}^{d}(T) \subset V_{\circ}(T)$ a subset corresponding to dashed corollas, $V_{\bullet}(T)$ the set of black vertices, and $E_{\circ}^{\bullet}(T)$ the set of internal edges of the type $\mathfrak{l}_{0}$. The isomorphism $\alpha_{T}$ between $\mathcal{U}_{T}$ and the latter product of manifolds with corners can be read from the metric graph via a simple procedure which we explain on the particular example. For the tree $T$ shown above the map $\alpha_{T}$ is defined by a formal extension of the domain of the following continuous man

$$
\begin{array}{rrrr}
(l,+\infty)^{2} \times(0, s) \times \quad C_{3}^{s t}(\mathbb{R}) \times \mathfrak{C}_{1}^{s t}(\mathbb{R}) \times \quad C_{2}^{s t}(\mathbb{R}) \times \mathfrak{C}_{3}^{s t}(\mathbb{R}) \times \mathfrak{C}_{2}^{s t}(\mathbb{R}) \times \mathfrak{C}_{1}^{s t}(\mathbb{R}) \times \quad & C_{2}^{s t}(\mathbb{R}) \\
\left(\tau_{1}, \tau_{2}\right) \times \quad \varepsilon \times\left(x^{\prime}, x^{\prime \prime}, x^{\prime \prime \prime}\right) \times\left(x_{1}=0\right) \times\left(t^{\prime}=\frac{-1}{\sqrt{2}}, t^{\prime \prime}=\frac{1}{\sqrt{2}}\right) \times\left(x_{2}, x_{4}, u\right) \times\left(x_{3}, x_{5}\right) \times\left(x_{6}=0\right) \times\left(x_{7}=\frac{-1}{\sqrt{2}}, x_{8}=\frac{1}{\sqrt{2}}\right) & \\
& \longrightarrow & \mathfrak{C}_{8}(\mathbb{R}) \\
& \longrightarrow\left(y_{1}, \ldots, y_{8}\right)
\end{array}
$$

with

$$
\begin{array}{ll}
y_{1}=\tau_{1} x^{\prime}+x_{1} & y_{2}=\tau_{1} x^{\prime \prime \prime}+x_{2} \\
y_{3}=\tau_{1} x^{\prime \prime}+\tau_{2} t^{\prime}+x_{3} & y_{4}=\tau_{1} x^{\prime \prime \prime}+x_{4} \\
y_{5}=\tau_{1} x^{\prime \prime}+\tau_{2} t^{\prime}+x_{5} & y_{7}=\tau_{1} x^{\prime \prime \prime}+u+\varepsilon x_{7} \\
y_{6}=\tau_{1} x^{\prime \prime}+\tau_{2} t^{\prime \prime}+x_{6}, & y_{8}=\tau_{1} x^{\prime \prime \prime}+u+\varepsilon x_{8}
\end{array}
$$

The boundary strata in $\mathcal{U}_{T}$ are given by allowing formally $\tau_{1}=+\infty, \tau_{2}=+\infty$ with $\tau_{1} / \tau_{2}=0$ and $\varepsilon=0$. Therefore, the main novelty comparing to the case of associahedra discussed in Sect. 2.1 comes from the dashed corollas decorated by a large parameter $\tau$.

3.2. Another configuration space model for $\operatorname{Mor}\left(\mathcal{A}_{\infty}\right)$. For a pair of subspaces $B \subsetneq A \subseteq[n]$ we consider

$$
\begin{array}{cccc}
\pi_{A, B}: \mathfrak{C}_{n}(\mathbb{R}) & \longrightarrow & C_{B}^{s t}(\mathbb{R}) \\
p & \longrightarrow \frac{p_{B}-x_{c}\left(p_{B}\right)}{\left\|p_{B}\right\|} & & \times p_{A, B}\|:=\| p_{A}\|\cdot\| p_{B} \|
\end{array}
$$

and then define a compactification $\sqrt{6} \widehat{\mathfrak{C}}_{\bullet}(\mathbb{R})$ as the closure of the following composition of embeddings,

$$
\mathfrak{C}_{n}(\mathbb{R}) \stackrel{\Psi_{n}}{\longrightarrow \pi_{A, B}} C_{n}^{s t}(\mathbb{R}) \times(0,+\infty) \prod_{\substack{B \subseteq A \subset[n] \\ \# B \geq 2}} C_{B}^{s t}(\mathbb{R}) \times(0,+\infty) \hookrightarrow \widetilde{C}_{n}^{s t}(\mathbb{R}) \times[0,+\infty] \prod_{\substack{B \subseteq A \subset[n] \\ \# B \geq 2}} \widetilde{C}_{B}^{s t}(\mathbb{R}) \times[0,+\infty]
$$

This new compactification affects only configurations tending to infinity: if, for a pair $B \subsetneq A \subseteq[n]$, the parameter $\left\|p_{A}\right\|$ tends to $+\infty$, then, for the parameter $\left\|p_{A, B}\right\|$ used in the above compactification to take a finite value $a \in \mathbb{R}$, one must have $\left\|p_{B}\right\|=a /\left\|p_{A}\right\| \rightarrow 0$. Put another way, if in the previous compactification (5) the boundary stratum

\footnotetext{
${ }^{5}$ We ordered factors $C_{\# \operatorname{In}(v)}(\mathbb{R})$ and $\mathfrak{C}_{\# \operatorname{In}(v)}(\mathbb{R})$ in the formula below in accordance with a natural "from top to the bottom" and "from left to the right" ordering of the vertices $v$ of the planar tree $T$. .

${ }^{6}$ We use the same symbol $\widehat{\mathfrak{C}}_{\bullet}(\mathbb{R})$ to denote this new compactification as it has the same combinatoric of the boundary strata as the model in the previous subsection; we define essentially here a different manifold structure on the same set theoretic operad $\widehat{\mathfrak{C}}_{\bullet}(\mathbb{R})$.
} 
at "infinity" was given by, say, $k$ groups of points, $\left\{I_{i}\right\}_{i \in[k]}$, going far away from each other in such a way that each group $I_{i}$ has a finite size $a_{i} \in \mathbb{R}$, now that size should decrease as $a_{i} /\left\|p_{A}\right\|$ as points go to infinity, i.e. the points in each group $I_{i}$ gradually collapse to a single point in the limit.

As an illustration of this "renormalized" embedding formula, let us consider in detail the case $n=3$ :

$$
\begin{aligned}
& \begin{array}{lllllll}
\mathfrak{C}_{3}(\mathbb{R}) & \longrightarrow & \widetilde{C}_{3}^{s t}(\mathbb{R}) \times[0,+\infty] & \times & \widetilde{C}_{12}^{s t}(\mathbb{R}) \times[0,+\infty] & \times & \widetilde{C}_{23}^{s t}(\mathbb{R}) \times[0,+\infty]
\end{array} \\
& p=\left\{x_{1}<x_{2}<x_{3}\right\} \quad \rightarrow \quad\left(\frac{p-x_{c}(p)}{\|p\|},\|p\|\right) \quad\left\|p_{[3], 12}\right\|:=\|p\| \cdot\left\|p_{12}\right\| \quad\left\|p_{[3], 23}\right\|:=\|p\| \cdot\left\|p_{23}\right\| .
\end{aligned}
$$

The codimension 1 boundary strata of $\widehat{\mathfrak{C}}_{3}(\mathbb{R})$ decomposes into strata determined by various limit values of the parameters $\|p\|,\left\|p_{[3], 12}\right\|$ and $\left\|p_{[3], 12}\right\|$ in a close analogy to the case (55):

(a) the stratum, $\overbrace{1}^{\infty} \simeq \mathfrak{C}_{1}(\mathbb{R}) \times C_{3}^{s t}(\mathbb{R})$, is given by $\|p\|=\left\|p_{[3], 12}\right\|=\left\|p_{[3], 23}\right\|=0$; it represents the limit configurations in which all three points collapse into a single point in $\mathfrak{C}_{1}$ in such a way that the ratio $\frac{p-x_{c}(p)}{\|p\|}$ gives in the limit a well-defined point in $C_{3}^{s t}(\mathbb{R})$; any point in this boundary stratum can be obtained as the $\lambda \rightarrow 0$ limit of a configuration $\left(\lambda x_{1}, \lambda x_{2}, \lambda x_{3}\right) \in \mathfrak{C}_{3}(\mathbb{R})$ for some fixed $\left(x_{1}, x_{2}, x_{3}\right) \in C_{3}^{s t}(\mathbb{R})$;

(b) the stratum,

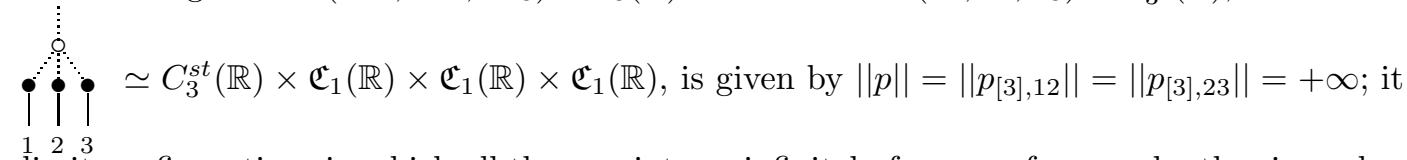
represents the limit configurations in which all three points go infinitely far away from each other in such a way that the ratio $\frac{p-x_{c}(p)}{\|p\|}$ is well-defined giving in the limit a point in $C_{3}^{s t}(\mathbb{R})$; any point in this boundary stratum can be obtained as the $\lambda \rightarrow+\infty$ limit of a configuration $\left(\lambda x_{1}, \lambda x_{2}, \lambda x_{3}\right) \in \mathfrak{C}_{3}(\mathbb{R})$ for some fixed $\left(x_{1}, x_{2}, x_{3}\right) \in C_{3}^{s t}(\mathbb{R})$

(c) the stratum, it represents the limit configurations in which the point 1 goes infinitely far away from the points 2 and 3 in such a way that the ratio $\frac{p-x_{c}(p)}{\|p\|}$ and the product $\|p\| \cdot\left\|p_{23}\right\|$ are well-defined elements of $\widetilde{C}_{3}^{s t}(\mathbb{R})$ and $(0,+\infty)$ respectively; any point in this boundary stratum can be obtained as the $\lambda \rightarrow+\infty$ limit of a configuration $\left(-\lambda, \lambda-\lambda^{-1} \Delta, \lambda+\lambda^{-1} \Delta\right) \in \mathfrak{C}_{3}(\mathbb{R})$ for some $\Delta \in \mathbb{R}$ as

$$
\begin{gathered}
\frac{p-x_{c}(p)}{\|p\|}=\frac{\left(-\frac{4}{3} \lambda, \frac{2}{3} \lambda-\lambda^{-1} \Delta, \frac{2}{3} \lambda+\lambda^{-1} \Delta\right)}{\sqrt{\frac{16}{9} \lambda^{2}+\left(\frac{2}{3} \lambda-\lambda^{-1} \Delta\right)^{2}+\left(\frac{2}{3} \lambda+\lambda^{-1} \Delta\right)^{2}}} \stackrel{\lambda \rightarrow+\infty}{\longrightarrow}\left(-\frac{\sqrt{2}}{\sqrt{3}}, \frac{1}{\sqrt{6}}, \frac{1}{\sqrt{6}}\right) \\
\left\|p_{[3], 23}\right\|=\|p\| \cdot\left\|p_{[3], 23}\right\| \quad=\quad \sqrt{\frac{16}{9} \lambda^{2}+\left(\frac{2}{3} \lambda-\lambda^{-1} \Delta\right)^{2}+\left(\frac{2}{3} \lambda+\lambda^{-1} \Delta\right)^{2}} \cdot \sqrt{2} \lambda^{-1} \Delta \\
\stackrel{\lambda \rightarrow+\infty}{\longrightarrow} \frac{4}{\sqrt{3}} \Delta \in(0,+\infty) .
\end{gathered}
$$

Thus this stratum consists of limit configurations in which the point $x_{1}$ goes far away from the points $x_{2}$ and $x_{3}$, and simultaneously, the points $x_{2}$ and $x_{3}$ approach each other with the speed given by $\|p\|^{-1}$ so that the product $\|p\| \cdot\left\|p_{23}\right\|$ is a well-defined finite number.

(d) the stratum,

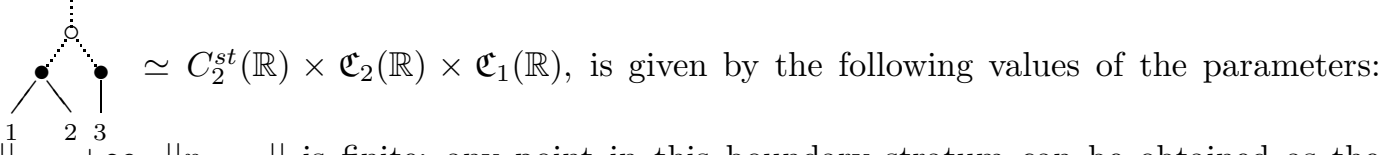
$\|p\|=\left\|p_{[3], 23}\right\|^{1}={ }^{2}+\infty,\left\|p_{[3], 12}\right\|$ is finite; any point in this boundary stratum can be obtained as the $\lambda \rightarrow+\infty$ limit of a configuration $\left(-\lambda-\lambda^{-1} \Delta,-\lambda+\lambda^{-1} \Delta, \lambda\right) \in \mathfrak{C}_{3}(\mathbb{R})$ for some $\Delta \in \mathbb{R}$,

(e) the stratum

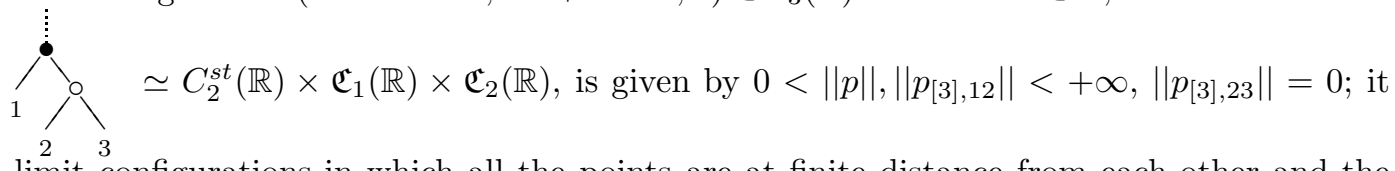
represents the limit ${ }^{2}$ configurations in which all the points are at finite distance from each other and the 
points 2 and 3 collapse into a single point in $\mathfrak{C}_{2}(\mathbb{R})$; any point in this boundary stratum can be obtained as the $\lambda \rightarrow 0$ limit of a configuration $(-1,1-\lambda \Delta, 1+\lambda \Delta, 1) \in \mathfrak{C}_{3}(\mathbb{R})$ for some $\Delta \in \mathbb{R}$

(f) the stratum,

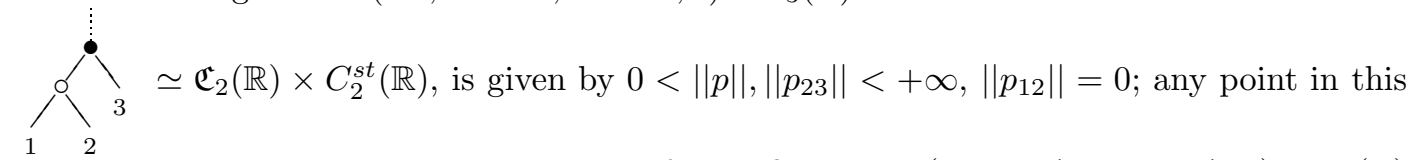
boundary stratum can be obtained as the $\lambda \rightarrow 0$ limit of a configuration $(-1-\lambda \Delta,-1+\lambda \Delta, 1) \in \mathfrak{C}_{3}(\mathbb{R})$ for some $\Delta \in \mathbb{R}$.

It is important to notice the following difference between the compactification formulae (5) and (10):

- consider limit configurations in which the points $x_{2}$ and $x_{3}$ go far away from the point $x_{1}$ while keeping a finite distance, $\left\|p_{23}\right\|$, between themselves; in the first compactification formula such limit configurations fill in the stratum as $\|p\|,\left\|p_{12}\right\| \rightarrow+\infty$ while $\left\|p_{23}\right\|$ stays finite; in the second compactification formula all such configurations tend to one and the same point in the boundary represented by the graph as in this case all three parameters, $\|p\|,\|p\| \cdot\left\|p_{23}\right\|$ and $\|p\| \cdot\left\|p_{12}\right\|$, tend to $+\infty$ and the limit $i$

point $p /\|p\|$ in $\widetilde{C}_{3}^{s t}$ consists of only two different points (rather than of three ones as in the case of a generic point in the stratum

- consider now limit configurations in which the point $x_{1}$ goes far away from the points $x_{2}$ and $x_{3}$, and simultaneously, the points $x_{2}$ and $x_{3}$ approach each other with the speed $\sim\|p\|^{-1}$ so that the product $\|p\| \cdot\left\|p_{23}\right\|$ is a well-defined finite number; in the second compactification such limit configurations fill in the stratum but in the first compactification all such configurations tend to one and the same point in the boundary given by the graph

Using metric graphs we can make the second topological compactification, $\widehat{\mathfrak{C}}_{\bullet}(\mathbb{R})$, into a smooth manifold with corners by constructing a coordinate chart, $\mathcal{U}_{T}$, near the boundary stratum corresponding to an arbitrary tree $T \in \mathcal{M o r}\left(A_{\infty}\right)$ as follows:

(i) Associate to $T$ a metric graph, $T_{\text {metric }}$, by assigning

(a) to every internal edge of $T$ of the form $[$ a small positive real number $\varepsilon \ll+\infty$;

(b) to every (if any) white vertex of a dashed corolla in $T$ a large positive real number $\tau \gg 0$, and to its every incoming edge a small parameter $\tau^{-1}$,

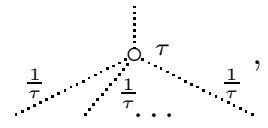

(c) to every (if any) two vertex subgraph of $T_{\text {metric }}$ of the form $\stackrel{\overbrace{1}^{\tau_{1}}}{\tau_{1}^{-1}}$ an inequality $\tau_{1} \gg \tau_{2} \gg 0$. This can be understood as a relation $\tau_{2}=\varepsilon_{12} \tau_{1}$ for some small parameter $\varepsilon_{12}$.

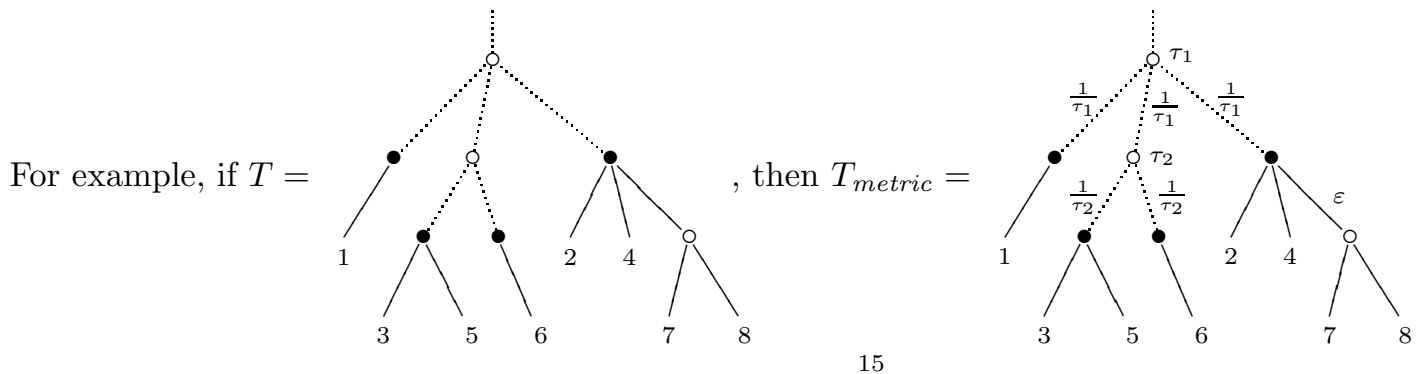


with $\tau_{1}, \tau_{2}=\varepsilon_{12} \tau_{1} \in(l,+\infty)$ and $\varepsilon_{12}, \varepsilon \in(0, s)$ for some large $l \gg 0$ and small $s \ll+\infty$ real numbers.

(ii) Choose an equivariant section, $s: \mathfrak{C}_{n}(\mathbb{R}) \rightarrow \operatorname{Conf}_{n}(\mathbb{R})$, of the natural projection $\operatorname{Conf}_{n}(\mathbb{R}) \rightarrow \mathfrak{C}_{n}(\mathbb{R})$ as well as an arbitrary smooth structure on its image, $\mathfrak{C}_{n}^{s t}(\mathbb{R}):=s\left(\mathfrak{C}_{n}(\mathbb{R})\right)$, which is called the space of configurations in the standard position; for example, $\mathfrak{C}_{n}^{s t}(\mathbb{R})$ can be chosen to be the subspace of $C_{0 n f}(\mathbb{R})$ satisfying the condition $\sum_{i=1}^{n} x_{i}=0 ;$ in particular, $\mathfrak{C}_{1}^{s t}(\mathbb{R})=0 \in \mathbb{R}$.

(iii) the required coordinate chart $\mathcal{U}_{T} \subset \overline{\mathfrak{C}}_{n}(\mathbb{R})$ is, by definition, isomorphic to the manifold with corners,

$$
(l,+\infty]^{\# V_{0}^{d}(T)} \times[0, s)^{\# E_{0}^{*}(T)} \times \prod_{v \in V_{0}(T)} C_{\# \operatorname{In}(v)}(\mathbb{R}) \times \prod_{v \in V_{\bullet}(T)} \mathfrak{C}_{\# \operatorname{In}(v)}(\mathbb{R})
$$

where $V_{\circ}(T)$ is the set of white vertices of $T, V_{\circ}^{d}(T) \subset V_{\circ}(T)$ a subset corresponding to dashed corollas, $V_{\bullet}(T)$ the set of black vertices, and $E_{\circ}^{\bullet}(T)$ the set of internal edges of the type $\mathfrak{j}_{0}$. The isomorphism $\alpha_{T}$ between $\mathcal{U}_{T}$ and the latter product of manifolds with corners can be read from the metric graph via a simple procedure which we explain on the particular example. For the tree $T$ shown above the map $\alpha_{T}$ is defined by a formal extension of the domain of the following continuous man?

$$
\begin{aligned}
(l,+\infty)^{2} \times(0, s) \times \quad C_{3}^{s t}(\mathbb{R}) \times \mathfrak{C}_{1}^{s t}(\mathbb{R}) \times \quad C_{2}^{s t}(\mathbb{R}) \times \mathfrak{C}_{3}^{s t}(\mathbb{R}) \times \mathfrak{C}_{2}^{s t}(\mathbb{R}) \times \mathfrak{C}_{1}^{s t}(\mathbb{R}) \times & C_{2}^{s t}(\mathbb{R}) \\
\left(\tau_{1}, \tau_{2}\right) \times \varepsilon \times\left(x^{\prime}, x^{\prime \prime}, x^{\prime \prime \prime}\right) \times\left(x_{1}=0\right) \times\left(t^{\prime}=\frac{-1}{\sqrt{2}}, t^{\prime \prime}=\frac{1}{\sqrt{2}}\right) \times\left(x_{2}, x_{4}, u\right) \times\left(x_{3}, x_{5}\right) \times\left(x_{6}=0\right) \times\left(x_{7}=\frac{-1}{\sqrt{2}}, x_{8}=\frac{1}{\sqrt{2}}\right) & \\
& \longrightarrow \\
& \mathfrak{C}_{8}(\mathbb{R}) \\
& \left(y_{1}, \ldots, y_{8}\right)
\end{aligned}
$$

with

$$
\begin{array}{ll}
y_{1}=\tau_{1} x^{\prime}+\frac{1}{\tau_{1}} x_{1} & y_{2}=\tau_{1} x^{\prime \prime \prime}+\frac{1}{\tau_{1}} x_{2} \\
y_{3}=\tau_{1} x^{\prime \prime}+\frac{1}{\tau_{1}}\left(\tau_{2} t^{\prime}+\frac{1}{\tau_{2}} x_{3}\right) & y_{4}=\tau_{1} x^{\prime \prime \prime}+\frac{1}{\tau_{1}} x_{4} \\
y_{5}=\tau_{1} x^{\prime \prime}+\frac{1}{\tau_{1}}\left(\tau_{2} t^{\prime}+\frac{1}{\tau_{2}} x_{5}\right) & y_{7}=\tau_{1} x^{\prime \prime \prime}+\frac{1}{\tau_{1}}\left(u+\varepsilon x_{7}\right) \\
y_{6}=\tau_{1} x^{\prime \prime}+\frac{1}{\tau_{1}}\left(\tau_{2} t^{\prime \prime}+\frac{1}{\tau_{2}} x_{6}\right), & y_{8}=\tau_{1} x^{\prime \prime \prime}+\frac{1}{\tau_{1}}\left(u+\varepsilon x_{8}\right)
\end{array}
$$

The boundary strata in $\mathcal{U}_{T}$ are given by allowing formally $\tau_{1}=+\infty, \tau_{2}=+\infty$ with $\tau_{1} / \tau_{2}=0$ and/or $\varepsilon=0$. Therefore, the only novelty comparing to the case of associahedra discussed in Sect. 2.1 comes from the dashed corollas decorated by a large parameter $\tau$; such a corolla tacitly assumes two rescaling operations: the first one is a magnification of the standard configuration used to decorate its vertex by the parameter $\tau$, and the second is a compression by the factor $\tau^{-1}$ of the standard configurations which correspond to all the corollas attached to its legs.

It is this second smooth structure on $\widehat{\mathfrak{C}}_{\bullet}(\mathbb{R})$ (and its higher dimensional analogs, $\widehat{\mathfrak{C}}_{\bullet}\left(\mathbb{R}^{d}\right)$ and $\widehat{\mathfrak{C}}_{\bullet}(\mathbb{H})$, see below) which we shall be interested in applications. We have no interesting propagators to show in the case of smooth structures of the first type at present.

3.3. One more configuration space model for $\operatorname{Mor}\left(\mathcal{A}_{\infty}\right)$. Let $\mathbb{R}^{+}:=\{x \in \mathbb{R} \mid x>0\}$ and, for a finite set $A$, consider a configuration space,

$$
\operatorname{Conf}_{A}\left(\mathbb{R}^{+}\right)=\left\{A \hookrightarrow \mathbb{R}^{+}\right\}
$$

of all injections of $A$ into $\mathbb{R}^{+}$. The 1-dimensional Lie group $\mathbb{R}^{+}$acts on Conf $A(\mathbb{R})$ by dilations,

$$
\begin{gathered}
\operatorname{Conf}_{A}\left(\mathbb{R}^{+}\right) \times \mathbb{R}^{+} \longrightarrow c \operatorname{Conf}_{A}\left(\mathbb{R}^{+}\right) \\
\left(p=\left\{x_{i}\right\}_{i \in A}, \lambda\right) \longrightarrow \lambda p:=\left\{\lambda x_{i}\right\}_{i \in A}
\end{gathered}
$$

This action is free so that the quotient space (cf. (4)),

$$
\mathfrak{C}_{A}\left(\mathbb{R}^{+}\right):=\operatorname{Conf}_{A}\left(\mathbb{R}^{+}\right) / \mathbb{R}^{+},
$$

is a naturally oriented ( $\# A-1$ )-dimensional real manifold equipped with a smooth action of the permutation group $\operatorname{Aut}(A)$. The space $\mathfrak{C}_{1}\left(\mathbb{R}^{+}\right)$is a point and hence closed. For any $p \in \operatorname{Conf}{ }_{A}\left(\mathbb{R}^{+}\right)$we set $x_{\min }(p):=\inf _{i \in A} x_{i}$. Define the following section,

$$
C_{A}^{s t}\left(\mathbb{R}^{+}\right):=\left\{p \in \operatorname{Conf}_{A}\left(\mathbb{R}^{+}\right) \mid x_{\min }(p)=1 \text { and }\left|p-x_{\min }(p)\right|=1\right\}
$$

\footnotetext{
${ }^{7}$ We ordered factors $C_{\# \operatorname{In}(v)}(\mathbb{R})$ and $\mathfrak{C}_{\# \operatorname{In}(v)}(\mathbb{R})$ in the formula below in accordance with a natural "from top to the bottom" and "from left to the right" ordering of the vertices $v$ of the planar tree $T$. We have also thrown away factors $\mathfrak{C}_{1}^{s t}(\mathbb{R})$ as they are just single points identified with $0 \in \mathbb{R}$.
} 
of the natural projection $\operatorname{Conf}_{A}\left(\mathbb{R}^{+}\right) \rightarrow C_{A}(\mathbb{R})$. Then, for any finite set $A$ with $\# A \geq 2$, we have an equivariant isomorphism,

$$
\begin{aligned}
& \Psi_{A}: \mathfrak{C}_{A}\left(\mathbb{R}^{+}\right) \quad \longrightarrow \quad C_{A}^{s t}\left(\mathbb{R}^{+}\right) \times \mathbb{R}^{+} \quad \simeq C_{A}(\mathbb{R}) \times(0,+\infty) \\
& p \quad \longrightarrow \quad\left(\frac{p-x_{\min }(p)}{\left|p-x_{\min }(p)\right|}+1,\left|p-x_{\min }(p)\right|\right) .
\end{aligned}
$$

Next one can define a compactification, $\widehat{\mathfrak{C}}_{n}\left(\mathbb{R}^{+}\right)$, of the space $\mathfrak{C}_{n}\left(\mathbb{R}^{+}\right)$using either formulae analogous to (5D) or to (10); the face complex of $\widehat{\mathfrak{C}}_{n}\left(\mathbb{R}^{+}\right)$is precisely the operad $\mathcal{M o r}\left(\mathcal{A}_{\infty}\right)$.

3.4. Metric graphs and smooth structures on compactified configuration spaces. To save space-time below in this paper we devote this subsection to a formalization of the Kontsevich type construction of a smooth atlas on a compactified configuration space with the help of metric trees which was used in Sections 2.2 and $\mathbf{3 . 2}$. This subsection can be skipped as its only purpose is to give a rigorous (and obvious) definition of the words "by analogy to $\$ \mathbf{2 . 2}$ or to $\mathbf{3 . 2}$ ' which we use several times below.

All compactified configurations spaces we work with in this paper come equipped with a structure of topological (coloured) operad, $\bar{C}=\left\{\bar{C}_{n}\right\}_{n \geq 1}$ which, as an operad in the category of sets, is free, $\bar{C}=\mathcal{F} r e e\langle C\rangle$, and generated by an $\mathbb{S}$-space,

$$
C=\left\{C_{p}:=\operatorname{Conf}_{p}(\mathbb{V}) / G\right\}_{p \geq 1},
$$

such that each $C_{p}$ is the quotient of the configuration space of $p$ pairwise distinct numbered points in a subset 8 $\mathbb{V} \subset \mathbb{R}^{d}$ with respect to an action of a subgroup $G$ of the group $\mathbb{R}^{+} \ltimes \mathbb{R}^{d}$ (with $\mathbb{R}^{+}$acting on $\mathbb{R}^{d}$ by dilations) which preserves $\mathbb{V}$ and commutes with the natural action of $\mathbb{S}_{p}$ on $\operatorname{Conf} f_{p}\left(\mathbb{R}^{d}\right)$. As $\bar{C}=\mathcal{F}$ ree $\langle C\rangle$, each topological space $\bar{C}_{n}$ is canonically stratified,

$$
\bar{C}_{n}=\coprod_{T \in \mathcal{T}_{n}} C_{T} \quad\left(C_{T} \hookrightarrow \bar{C}_{n} \text { is continous }\right)
$$

into a disjoint union of cartesian products,

$$
C_{T}=\prod_{v \in V(T)} C_{\# \operatorname{In}(v)}
$$

Here $\mathcal{T}_{n}$ stands for the family of trees whose input legs are in a fixed bijection with $[n], V(T)$ stands for the set of vertices of a tree $T \in \mathcal{T}_{n}$, and, for a $v \in V(T)$, $\operatorname{In}(v)$ stands for its set of input half-edges. For example if $T$ is given by (2), then $C_{T} \simeq C_{3}^{\times 2} \times C_{2}^{\times 2}$.

In all cases of interest in this paper the structure of a smooth manifold with corners on $\bar{C}_{n}$ will be defined via an explicit construction of a coordinate chart $\mathcal{U}_{T}$ at each stratum $C_{T}$ with the help of an associated metric tree, $T_{\text {metric }}$, obtained from $T$

(i) by assigning to all vertices, $v$, of some fixed colour a large parameter $\tau \in \mathbb{R}^{+}$and simultaneously to all input legs of $v$ the small parameter $\tau^{-1}$, see (11), and

(ii) by assigning to all other internal edges a small parameter $\varepsilon$, see (3) for an example.

As a result every internal edge of $T_{\text {metric }}$ gets assigned a small parameter $\varepsilon$ or $\tau^{-1}$. To read the coordinate chart $\mathcal{U}_{T}$ from such a metric tree one has to choose a suitable $\mathbb{S}_{n}$-equivariant section,

$$
s: C_{n} \longrightarrow \operatorname{Conf}_{n}(\mathbb{V}) \subset \operatorname{Conf}_{n}\left(\mathbb{R}^{d}\right),
$$

of the natural projection $\operatorname{Conf}_{n}(\mathbb{V}) \rightarrow C_{n}$. The subspace $s\left(C_{\bullet}\right) \subset C_{0 n f}(\mathbb{R})$ is denoted by $C_{\bullet}^{s t}$ and called the space of standard configurations. Then one can use a natural action of $\mathbb{R}^{+} \ltimes \mathbb{V}$ on $\operatorname{Conf}_{n}\left(\mathbb{R}^{d}\right)$ to define a suitable translation map,

and a rescaling map,

$$
\begin{aligned}
T: \mathbb{V} \times C_{n}^{s t} & \longrightarrow \operatorname{Conf}_{n}(\mathbb{V}) \\
\left(z_{0}, p\right) & \longrightarrow T_{z_{0}}(p)
\end{aligned}
$$

$$
\begin{array}{ccc}
.: \mathbb{R}^{+} \times C_{n}^{s t} & \longrightarrow & \operatorname{Conf}_{n}(\mathbb{V}) \\
(\lambda, p) & \longrightarrow & \lambda . p .
\end{array}
$$

\footnotetext{
${ }^{8}$ Say, $\mathbb{V}$ can be the "upper half" of $\mathbb{R}^{d}$ with respect to the coordinate $x^{d}$.

${ }^{9} \mathrm{~A}$ smooth structure on the compactification (5) should be described with the help of metric corollas (9) without assigning to its input legs the small paramter $\tau^{-1}$; however, we never use such a smooth structure in applications and, therefore, exclude it from now on from the consideration in this paper.
} 
Having made choices for $C_{n}^{s t}$ and the maps $T_{z_{0}}$ and $\lambda_{\text {. }}$, one constructs out of a metric tree an open subset, $\mathcal{U}_{T}$, containing the boundary stratum $T$ together with a homeomorphism,

$$
\phi_{T}: \mathcal{U}_{T} \longrightarrow[0, \delta)^{m} \times \prod_{v \in V(T)} C_{\# I n(v)}^{s t},
$$

for some sufficiently small positive real number $\delta$ and natural number $m$. This homeomorphism together with a suitable choice of an $\mathbb{S}_{\bullet}$-equivariant structure of a smooth manifold with corners on each $C_{\bullet}^{s t}$ makes $\mathcal{U}_{T}$ itself into a smooth manifold with corners; the final step in the construction of a smooth atlas $\left\{\mathcal{U}_{T}\right\}$ on $\bar{C}$ • is to check smoothness of the transition functions at the non-empty intersections $\mathcal{U}_{T} \cap \mathcal{U}_{T^{\prime}}$ of the coordinate charts (which is often straighforward).

The construction of (13) is universal and is best explained in a particular example: if

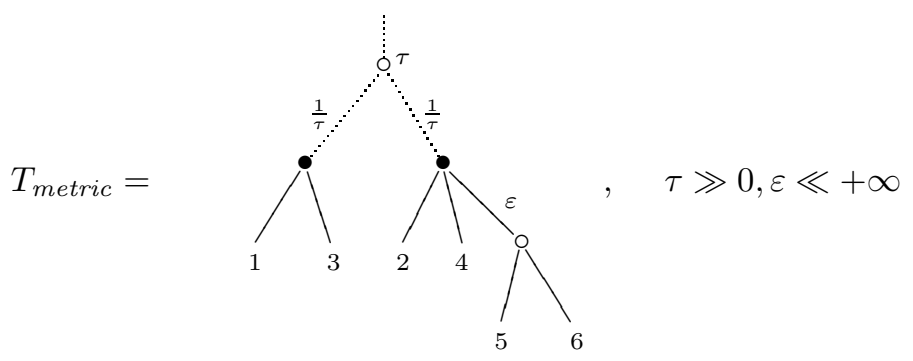

then $\mathcal{U}_{T} \cap C_{6}$ is given, by definition, by the image under projection $\operatorname{Conf}_{6}(\mathbb{V}) \rightarrow C_{6}$ of a subset consisting of all possible configurations, $\left\{z_{1}, \ldots, z_{6}\right\} \in \operatorname{Conf}_{6}(\mathbb{V})$, which can be obtained as follows

Step 1: Take an arbitrary standardly positioned configuration, $p^{(1)}=\left(z^{\prime}, z^{\prime \prime}\right) \in C_{2}^{s t}$ and apply $\tau$-rescaling, $p^{(1)} \rightarrow$ $\tau \cdot p^{(1)}=:\left(\tau \cdot z^{\prime}, \tau \cdot z^{\prime \prime}\right)$

Step 2: Take arbitrary standard configurations, $p^{(2)}=\left(z_{1}^{s t}, z_{3}^{s t}\right) \in C_{2}^{s t}$ and $p^{(3)}=\left(z_{2}^{s t}, z_{4}^{s t}, z^{\prime \prime \prime}\right) \in C_{3}^{s t}, \tau^{-1}$-rescale them,

$$
\begin{aligned}
& p^{(2)}=\left(z_{1}^{s t}, z_{3}^{s t}\right) \longrightarrow \tau^{-1} \cdot p^{(2)}=:\left(z_{1}, z_{3}\right), \\
& p^{(3)}=\left(z_{2}^{s t}, z_{4}^{s t}, z^{\prime \prime \prime}\right) \longrightarrow \tau^{-1} \cdot p^{(3)}=:\left(z_{2}, z_{4}, z^{\prime \prime \prime \prime}\right),
\end{aligned}
$$

and then place the results at the points $\tau \cdot z^{\prime}$ and $\tau \cdot z^{\prime}$ respectively, i.e. consider a configuration

$$
T_{\tau \cdot z^{\prime}}\left(\tau^{-1} \cdot p^{(2)}\right) \coprod T_{\tau \cdot z^{\prime \prime}}\left(\tau^{-1} \cdot p^{(3)}\right)=:\left(z_{1}, z_{3}, z_{2}, z_{4}, z^{\prime \prime \prime \prime \prime \prime}\right) \in \operatorname{Conf}_{5}(\mathbb{V}) ;
$$

Step 3: Finally, take an arbitrary standardly positioned configuration, $p^{(4)}=\left(z_{5}^{s t}, z_{6}^{s t}\right) \in C_{2}^{s t}$, apply $\varepsilon \tau^{-1}$-rescaling, $p^{(4)} \rightarrow \varepsilon \tau^{-1} \cdot p^{(4)}$, and place the result into the point $z^{\prime \prime \prime \prime \prime \prime}$. i.e. consider $T_{z^{\prime \prime \prime \prime \prime \prime}}\left(\varepsilon \tau^{-1} \cdot p^{(4)}\right)=:\left(z_{5}, z_{6}\right)$.

If the constructed continuous map,

$$
\phi_{T}:(0, \delta)^{2} \times\left(C_{2}^{s t}\right)^{\times 3} \times C_{3}^{s t} \rightarrow \operatorname{Conf}_{6}(\mathbb{V})
$$

is an injection for a sufficiently small $\delta \in \mathbb{R}^{+}$(and this will be the case in all cases of interest in this paper), then its image gives us the desired smooth coordinate chart $\mathcal{U}_{T}$; the boundary stratum $C_{T}$ is given in this chart by setting formally the small parameters $\varepsilon, \tau^{-1} \in(0, \delta)$ to zero.

The above construction is applicable to all operads, $\bar{C}$, of compactified configuration spaces studied in this paper. In each concrete case we have to specify only three things,

(i) an association $T \rightarrow T_{\text {metric }}$,

(ii) a definition of the space, $C_{n}^{s t} \subset \operatorname{Conf}_{n}(\mathbb{V})$, of standard positions, and

(iii) a rescaling map, . : $\mathbb{R}^{+} \times C_{n}^{s t} \rightarrow \operatorname{Conf}_{n}(\mathbb{V})$, and a translation map $T: \mathbb{V} \times C_{n}^{s t} \rightarrow \operatorname{Conf}_{n}(\mathbb{V})$.

the rest of the construction of a smooth atlas goes along the lines formalized in this subsection. 


\section{Kontsevich configuration spaces and open-closed homotopy algebras}

\subsection{Fulton-MacPherson compactification of points on the complex plane Ko2. Let}

$$
\operatorname{Conf}_{n}(\mathbb{C}):=\left\{z_{1}, \ldots, z_{n} \in \mathbb{C} \mid z_{i} \neq z_{j} \text { for } i \neq j\right\}
$$

be the configuration space of $n$ pairwise distinct points on the complex plane $\mathbb{C}$. The space $C_{n}(\mathbb{C})$ is a smooth $(2 n-3)$-dimensional real manifold defined as the orbit space [Ko2],

$$
C_{n}(\mathbb{C}):=\operatorname{Conf}_{n}(\mathbb{C}) / G_{(3)},
$$

with respect to the following action of a real 3-dimensional Lie group,

$$
G_{(3)}=\left\{z \rightarrow a z+b \mid a \in \mathbb{R}^{+}, b \in \mathbb{C}\right\} .
$$

The space $C_{2}(\mathbb{C})$ is homeomorphic to the circle $S^{1}$ and hence is compact. The compactification, $\bar{C}_{n}(\mathbb{C})$, of $C_{n}(\mathbb{C})$ for $n \geq 3$ can be defined $\mathrm{Ko} 2, \mathrm{Ga}$ ) as the closure of an embedding,

$$
\begin{aligned}
& C_{n}(\mathbb{C}) \quad \longrightarrow \quad(\mathbb{R} / 2 \pi \mathbb{Z})^{n(n-1)} \times \quad\left(\mathbb{R} \mathbb{P}^{2}\right)^{n(n-1)(n-2)} \\
& \left(z_{1}, \ldots, z_{n}\right) \longrightarrow \prod_{i \neq j} \operatorname{Arg}\left(z_{i}-z_{j}\right) \times \prod_{i \neq j \neq k \neq i}\left[\left|z_{i}-z_{j}\right|:\left|z_{j}-x_{k}\right|:\left|z_{i}-z_{k}\right|\right]
\end{aligned}
$$

The space $\bar{C}_{n}(\mathbb{C})$ is a smooth (naturally oriented) manifold with corners. Its codimension 1 strata is given by

$$
\partial \bar{C}_{n}(\mathbb{C})=\bigsqcup_{\substack{A \subset[n] \\ \# A \geq 2}} C_{n-\# A+1}(\mathbb{C}) \times C_{\# A}(\mathbb{C})
$$

where the summation runs over all possible proper subsets of $[n]$ with cardinality $\geq 2$. Geometrically, each such stratum corresponds to the $A$-labeled elements of the set $\left\{z_{1}, \ldots, z_{n}\right\}$ moving very close to each other. If we represent $\bar{C}_{n}(\mathbb{C})$ by the symmetric $n$-corolla of degree $3-2 n$

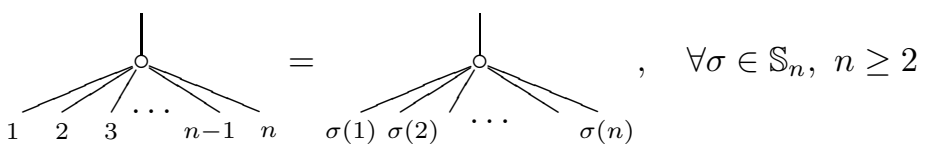

then the boundary operator in the associated face complex of $\bar{C} \bullet(\mathbb{C})$ takes a familiar form

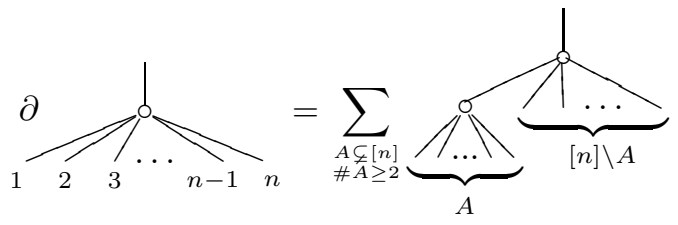

implying the following useful observation.

4.1.1. Proposition [GJ]. The face complex of the family of compactified configurations spaces, $\left\{\bar{C}_{n}(\mathbb{C})\right\}_{n \geq 2}$, has a structure of a dg free non-unital pseudo-operad canonically isomorphic to the operad, $L_{\infty}\{1\}$, of strong homotopy Lie algebras with degree shifted by on 11 .

4.1.2. Smooth atlas on $\bar{C}_{\bullet}(\mathbb{C})$. The coordinate chart $\mathcal{U}_{T}$ near the boundary stratum in $\bar{C} \cdot(\mathbb{C})$ corresponding to an arbitrary tree $T$ built from corollas (14) is constructed as in Section $\mathbf{2 . 2}$ by associating to $T$ a metric tree $T_{\text {metric }}$ whose every internal edge is assigned a small positive real number and whose every vertex, $v$, is decorated with an element of $C_{\# I n(v)}^{s t}(\mathbb{C})$ which is defined as the subset of $\operatorname{Conf}_{\# I n(v)}(\mathbb{C})$ consisting of all configurations $\left(z_{1}, \ldots, z_{\# \operatorname{In}(v)}\right)$ satisfying two conditions, $\sum_{i=1}^{\# \operatorname{In}(v)} z_{i}=0$ and $\sum_{i=1}^{\# I n(v)}\left|z_{i}\right|^{2}=1$. The rescaling (resp. translation) map on $C_{\bullet}^{s t}(\mathbb{C})$ is defined to be the ordinary dilation (resp. translation) map, see Remark $\mathbf{3 . 4}$.

\footnotetext{
${ }^{10}$ We prefer working with cochain complexes, and hence always adopt gradings accordingly.

${ }^{11}$ Denote the endomorphism operad, $\mathcal{E} n d_{\mathbb{K}[m]}$, of the one 1-dimensional graded vector space $\mathbb{K}[m]$ by $\{m\}$. Then for any dg operad $P$ the tensor product $P \otimes\{m\}=: P\{m\}$ is again an operad whose representations in a graded vector space $V$ are in one-to-one correspondence with representations of the operad $P$ in $V[m]$. Therefore, the association $P \rightarrow P\{m\}$ is a kind of degree shifting in the world of operads
} 
4.1.3. An equivalent construction of the compactification $\bar{C} \cdot(\mathbb{C})[\mathrm{AT}]$. Let $\operatorname{Conf}_{A}(\mathbb{C})$ stand for the space of immersions, $A \hookrightarrow \mathbb{C}$, of a finite non-empty set $A$ into the complex plane and $\widetilde{\operatorname{Conf}_{A}}(\mathbb{C})$ for the space of all possible maps. We define $C_{A}(\mathbb{C})=\operatorname{Conf}_{A}(\mathbb{C}) / G_{(3)}$ and, for a configuration $p=\left\{z_{i}\right\}_{i \in A} \in \operatorname{Conf}_{A}(\mathbb{C})$, we set,

$$
z_{c}(p):=\frac{1}{\# A} \sum_{i \in A} z_{i}, \quad\left|p-z_{c}(p)\right|:=\sqrt{\sum_{i \in A}\left|z_{i}-z_{c}(p)\right|^{2}} .
$$

Recall that $C_{A}(\mathbb{C})$ can be equivariantly identified with

$$
C_{A}^{s t}(\mathbb{C})=\left\{p \in \operatorname{Conf}_{n}(\mathbb{C})\left|z_{c}(p)=0,\right| p-z_{c}(p) \mid=1\right\}
$$

Let us also consider a space,

$$
\widetilde{C}_{A}^{s t}(\mathbb{C})=\left\{p \in \widetilde{\operatorname{Conf}}_{A}(\mathbb{C})\left|z_{c}(p)=0,\right| p-z_{c}(p) \mid=1\right\},
$$

which is a compact $(2 \# A-3)$-dimensional manifold with boundary. The compactification $\bar{C} \bullet(\mathbb{C})$ can be defined as the closure of an embedding,

$$
C_{n}(\mathbb{C}) \stackrel{\prod \pi_{A}}{\longrightarrow} \prod_{\substack{A \subseteq[n] \\ \# \bar{A} \geq 2}} C_{A}(\mathbb{C}) \stackrel{\simeq}{\longrightarrow} \prod_{\substack{A \subseteq[n] \\ \# A \geq 2}} C_{A}^{s t}(\mathbb{C}) \hookrightarrow \prod_{\substack{A \subseteq[n] \\ \# A \geq 2}} \widetilde{C}_{A}^{s t}(\mathbb{C}) .
$$

where the product runs over all possible subsets $A$ of $[n]$ with $\# A \geq 2$, and

$$
\begin{aligned}
& \pi_{A}: \quad C_{n}(\mathbb{C}) \quad \longrightarrow \quad C_{A}(\mathbb{C}) \\
& p=\left\{z_{i}\right\}_{i \in[n]} \longrightarrow p_{A}:=\left\{z_{i}\right\}_{i \in A}
\end{aligned}
$$

is the natural forgetful map.

4.1.4. Higher dimensional version. One sets, for $d \geq 2$,

$$
C_{n}\left(\mathbb{R}^{d}\right):=\frac{\left\{p_{1}, \ldots, p_{n} \in \mathbb{R}^{d} \mid p_{i} \neq p_{j} \text { for } i \neq j\right\}}{G_{(k+1)}},
$$

where $G_{(d+1)}:=\left\{p \rightarrow \lambda p+\nu \mid \lambda \in \mathbb{R}^{+}, \nu \in \mathbb{R}^{d}\right\}$. The map

$$
\begin{aligned}
& C_{2}\left(\mathbb{R}^{d}\right) \quad \longrightarrow \quad S^{d-1} \\
& \left(p_{1}, p_{2}\right) \quad \longrightarrow \quad \frac{p_{1}-p_{2}}{\left|p_{1}-p_{2}\right|}
\end{aligned}
$$

is an isomorphism so that $C_{2}\left(\mathbb{R}^{d}\right)$ is compact. For $n \geq 3$ the compactified configuration space $\bar{C}_{n}\left(\mathbb{R}^{d}\right)$ is defined as the closure of an embedding

$$
\begin{array}{ccccc}
C_{n}\left(\mathbb{R}^{d}\right) & \longrightarrow & \left(S^{d-1}\right)^{n(n-1)} & \times & \left(\mathbb{R}^{2}\right)^{n(n-1)(n-2)} \\
\left(p_{1}, \ldots, p_{n}\right) & \longrightarrow & \prod_{i \neq j} \frac{p_{i}-p_{j}}{\left|p_{i}-p_{j}\right|} \times & \times & \prod_{i \neq j \neq k \neq i}\left[\left|p_{i}-p_{j}\right|:\left|p_{j}-p_{k}\right|:\left|p_{i}-p_{k}\right|\right]
\end{array}
$$

The face complex of $\left\{\bar{C}_{n}\left(\mathbb{R}^{d}\right)\right\}_{n \geq 2}$ has a structure of a dg free operad canonically isomorphic to the operad of $\mathcal{A}_{\infty}$-algebras for $d=1$, and, for $d \geq 2$, to the operad, $\mathcal{L}_{\infty}\{d-1\}$, of strong homotopy Lie algebras with degree shifted by $d-1$ [GJ]. If $d$ is odd, then the action of an element $\sigma \in \mathbb{S}_{n}$ on $C_{n}\left(\mathbb{R}^{d}\right)$ preserves its natural orientation if the permutation $\sigma$ is even and reverses the orientation if $\sigma$ is odd. Therefore, the generating corollas (14) of the operad $\bar{C} \bullet\left(\mathbb{R}^{d}\right)$ are symmetric for $d$ even, and skewsymmetric for $d$ odd.

\subsection{Kontsevich's compactification of points in the upper half plane [Ko2]. Let}

$$
\operatorname{Conf}_{n, m}(\mathbb{H}):=\left\{z_{1}, \ldots, z_{n} \in \mathbb{H}, x_{\overline{1}}, \ldots, x_{\bar{m}} \in \mathbb{R} \subset \partial \overline{\mathbb{H}} \mid z_{i} \neq z_{j}, x_{\bar{i}} \neq x_{\bar{j}} \text { for } i \neq j\right\}
$$

be the configuration space of $n+m$ pairwise distinct points on the closed upper half plane $\overline{\mathbb{H}}$. For future reference we also define,

$$
\widetilde{\operatorname{Conf}}_{n, m}(\mathbb{H}):=\left\{z_{1}, \ldots, z_{n} \in \overline{\mathbb{H}}, x_{\overline{1}}, \ldots, x_{\bar{m}} \in \mathbb{R} \subset \partial \overline{\mathbb{H}}\right\},
$$

where the condition on the points being distinct is dropped.

For any configuration $p=\left(z_{1}, \ldots, z_{n}, x_{\overline{1}}, \ldots, x_{\bar{m}}\right) \in \operatorname{Conf}_{n, m}(\mathbb{H})$ we set

$$
x_{c}(p):=\frac{1}{n+m}\left(\sum_{i=1}^{n} \Re\left(z_{i}\right)+\sum_{i=1}^{m} x_{i}\right), \quad \text { and } \quad\left|p-x_{c}(p)\right|:=\sqrt{\sum_{i=1}^{p}\left|z_{i}-x_{c}(p)\right|^{2}+\sum_{i=1}^{q}\left|x_{i}-x_{c}(p)\right|^{2}} .
$$


The Lie group $G_{(2)}$ acts freely on $\operatorname{Conf}_{n, m}(\mathbb{H})$ with the space of orbits,

$$
C_{n, m}(\mathbb{H}):=\operatorname{Conf}_{n, m}(\mathbb{H}) / G_{(2)}, \quad 2 n+m \geq 2,
$$

being a $(2 n+m-2)$-dimensional naturally oriented manifold. A compactification, $\bar{C}_{n, m}(\mathbb{H})$, of $C_{n, m}(\mathbb{H})$ has been defined in $[\mathrm{Ko}$ ] as the closure of an embedding

$$
\begin{array}{clc}
C_{n, m}(\mathbb{H}) & \longrightarrow & C_{2 n+m}(\mathbb{C}) \\
\left(z_{1}, \ldots, z_{n}, x_{\overline{1}}, \ldots, x_{\bar{m}}\right) & \longrightarrow & \left(z_{1}, \ldots, z_{n}, \overline{z_{1}}, \ldots, \overline{z_{n}}, x_{\overline{1}}, \ldots, x_{\bar{m}}\right)
\end{array} \bar{C}_{2 n+m}(\mathbb{C}) .
$$

The face complex of a disjoint union,

$$
\bar{C}(\mathbb{H}):=\bar{C} \bullet(\mathbb{C}) \bigsqcup \bar{C} \bullet, \bullet(\mathbb{H}),
$$

has a natural structure of a dg free 2-colored operad KaSt generated by degree $3-2 n$ corollas (14) representing $\bar{C}_{n}(\mathbb{C}), n \geq 2$, and degree $2-2 n-m$ corollas,

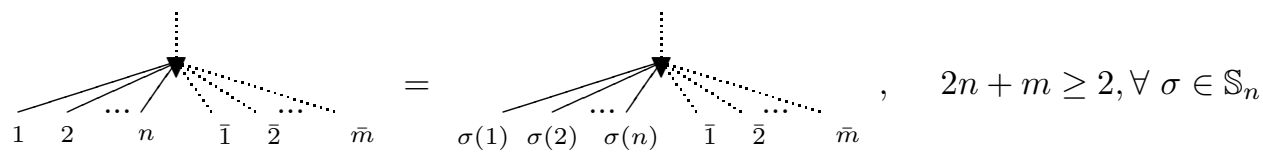

representing $\bar{C}_{n, m}(\mathbb{H})$. The boundary differential in the associated face complex is given on the generators by (15) and the following formula [Ko2, KaSt]

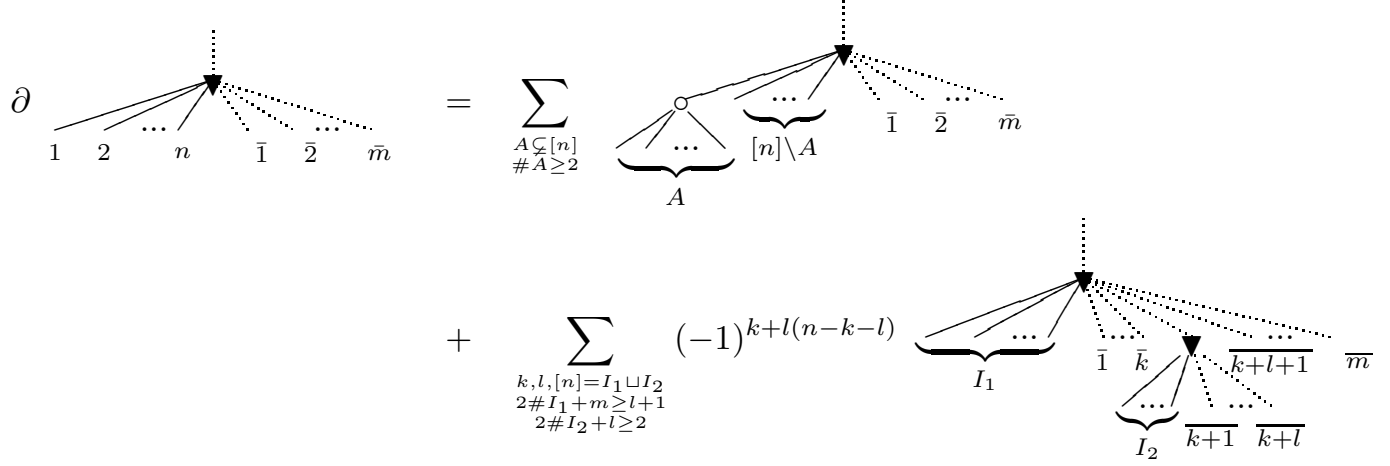

This operad was denoted in KaSt] by ${ }^{12} \mathcal{O C}_{\infty}$ and its representations in a pair of dg vector spaces $\left(X_{c}, X_{o}\right)$ were called open-closed homotopy algebras or OCHA for short.

It was shown in $\mathrm{Ho}$ that representations of $\mathcal{O C}_{\infty}$ are in one-to-one correspondence with degree one codifferentials in the tensor product, $\odot^{\bullet}\left(X_{c}[2]\right) \otimes \otimes \bullet\left(X_{o}[1]\right)$, of the free graded cocommutative coalgebra cogenerated by $X_{c}[2]$ and the free coalgebra cogenerated by $X_{o}[1]$. As $\mathcal{O C} \mathcal{C}_{\infty}$ is a free operad, its arbitrary representation, $\rho$, is uniquely determined by the values on the generators,

$$
\begin{aligned}
& \nu_{n}:=\rho(\overbrace{2}^{\infty} \overbrace{n-1}) \in \operatorname{Hom}\left(\odot^{n} X_{c}, X_{c}[3-2 n]\right), \quad n \geq 2,
\end{aligned}
$$

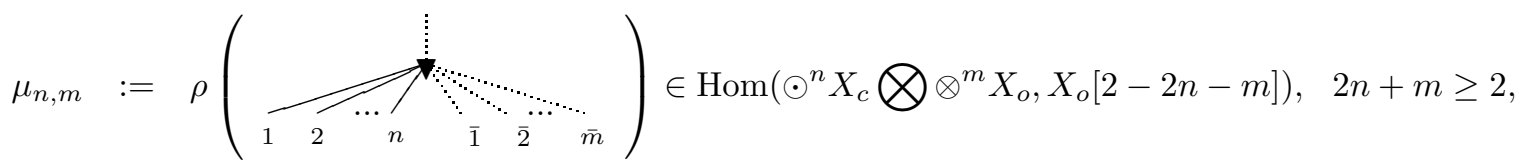

which satisfy quadratic relations given by the formulae for the differential $\partial$. One often denotes in this context the given differential in the dg space $X_{c}$ by $\nu_{1}$ and the one in $X_{o}$ by $\mu_{0,1}$.

\footnotetext{
${ }^{12}$ This notation may be misleading as this operad is neither a minimal resolution of some operad $\mathcal{O C}$ nor a cobar construction of some cooperad which is Koszul dual to a quadratic operad $\mathcal{O C}$.
} 
4.2.1. An interpretation of OCHA. Let $\operatorname{Coder}\left(\otimes^{\bullet}\left(X_{o}[1]\right),[],\right)$ be the Lie algebra of coderivations of the free coalgebra, $\otimes \bullet\left(X_{o}[1]\right)$, cogenerated by $X_{o}[1]$. We do not assume that coderivations preserve the co-unit so that MC elements, $\gamma$, in this Lie algebra describe, in general, non-flat $\mathcal{A}_{\infty}$-structures on $X_{o}$. We have an isomorphism of vector spaces,

$$
\operatorname{Coder}\left(\otimes{ }^{\bullet}\left(X_{o}[1]\right)\right)=\oplus_{m \geq 0} \operatorname{Hom}\left(\otimes^{m} X_{o}, X_{o}[1-m]\right) .
$$

It is not hard to check that a representation, $\rho$, of the $\mathrm{dg}$ operad $\mathcal{O C}_{\infty}$ in a pair of $\mathrm{dg}$ vector spaces $\left(X_{c}, X_{o}\right)$ is equivalent to the following data

(i) a $\mathcal{L}_{\infty}\{1\}$-algebra structure, $\nu=\left\{\nu_{n}: \odot^{n} X_{c} \rightarrow X_{c}[3-2 n]\right\}_{n \geq 1}$, in $X_{c}$, i.e. an ordinary $\mathcal{L}_{\infty}$-structure in $X_{c}[1]$

(ii) a $\mathcal{A}_{\infty}$-algebra structure, $\mu=\left\{\mu_{0, m}: \otimes^{m} X_{o} \rightarrow X_{c}[2-m]\right\}_{m \geq 1}$, in $X_{o}$; the associated MC element, $\mu$, of the Lie algebra $\left.\operatorname{Coder}\left(\otimes^{\bullet}\left(X_{o}[1]\right)\right),[],\right)$ makes the latter into a $d g$ Lie algebra with the differential $d_{\mu}:=[\mu$,$] ;$

(iii) a $\mathcal{L}_{\infty}$-morphism, $F$, from the $L_{\infty}$-algebra $\left(X_{c}[1], \nu\right)$ to the $\operatorname{dg}$ Lie algebra $\left(\operatorname{Coder}\left(\otimes \bullet\left(X_{o}[1]\right)\right),[],, d_{\mu}\right)$,

$$
F=\left\{F_{n}: \odot^{n} X_{c} \longrightarrow \operatorname{Coder}\left(\otimes \bullet\left(X_{o}[1]\right)\right)[1-2 n]\right\}_{n \geq 1}
$$

such that the composition

$$
\odot^{n} X_{c} \stackrel{F_{n}}{\longrightarrow} \operatorname{Coder}\left(\otimes \bullet\left(X_{o}[1]\right)\right)[1-2 n] \stackrel{\text { proj }}{\longrightarrow} \operatorname{Hom}\left(\otimes^{m} X_{o}, X_{o}[2-2 n-m]\right)
$$

coincides precisely with $\mu_{n, m}$ for any $n \geq 1, m \geq 0$.

If $\rho$ is an arbitrary representation of $\mathcal{O C}_{\infty}$ and $\gamma \in X_{c}$ is an arbitrary Maurer-Cartan element 13 ,

$$
\sum_{n \geq 0} \frac{1}{n !} \nu_{n}\left(\gamma^{\otimes n}\right)=0, \quad|\gamma|=2
$$

of the associated $\mathcal{L}_{\infty}$-algebra $\left(X_{c}, \nu\right)$, then the maps

$$
\mu_{m}:=\sum_{n \geq 1} \frac{\hbar^{n}}{n !} \mu_{n, m}\left(\gamma^{\otimes n} \otimes \ldots\right): \otimes^{m} X_{o} \longrightarrow X_{o}[[\hbar]][2-m], \quad m \geq 0,
$$

make the topological (with respect to the adic topology) vector space $X_{o}[[\hbar]]$ into a continuous and, in general, non-flat $\mathcal{A}_{\infty}$-algebra. Here $\hbar$ is a formal parameter, and $X_{c}\left[[\hbar]:=X_{c} \otimes \mathbb{K}[[\hbar]]\right.$.

4.2.2. Example. Kontsevich's formality construction [Ko2] gives a non-trivial representation of $\mathcal{O C}_{\infty}$ in the pair $\left(X_{c}:=\mathcal{T}_{\text {poly }}\left(\mathbb{R}^{d}\right), X_{o}:=C^{\infty}\left(\mathbb{R}^{d}\right)\right)$, consisting of the space of polyvector fields and the space of smooth functions on $\mathbb{R}^{d}$ for any $d$.

4.2.3. Smooth atlas on $\bar{C}(\mathbb{C}, \mathbb{H})$. A generic boundary stratum in $\bar{C}(\mathbb{H})=\bar{C} \bullet(\mathbb{C}) \amalg \bar{C} \bullet, \bullet(\mathbb{H})$ is given by a tree $T$ constructed from corollas (14) and (17). A smooth coordinate chart, $\mathcal{U}_{T}$, containing that boundary stratum is constructed as in Section $\mathbf{3 . 4}$ by associating to $T$ a metric tree $T_{\text {metric }}$ whose every (of any colour) internal edge is assigned a small positive real number $\varepsilon$ (i.e. there are no metric corollas type (11)). The spaces of standard configurations associated with o-vertices are set to be $C_{\bullet}^{s t}(\mathbb{C})$ and with $\mathbf{\nabla}$-vertices are set to be $C_{\bullet \bullet}^{s t}(\mathbb{H})$ which are, by definition, the subsets of Conf..$(\mathbb{H})$ consisting of all configurations $p$ satisfying two conditions, $x_{c}(p)=0$ and $\|p\|=1$. The rescaling and translation maps are defined to be the ordinary dilation, $z \rightarrow \lambda z$, and translation, $T_{z_{o}}: z \rightarrow z+z_{o}$, maps on both $C_{\bullet}^{s t}(\mathbb{C})$ and $C_{\bullet}^{s t}(\mathbb{H})$. The latter two groups of spaces can be equipped with arbitrary S-equivariant smooth structures.

\subsubsection{Example: Kontsevich eye.}

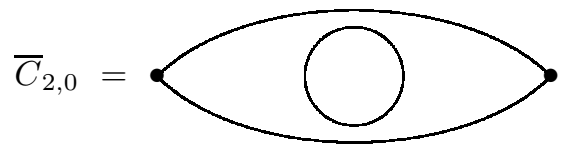

The codimension 1 boundary splits into the union of three strata: the inner circle $C_{2}(\mathbb{C})$ ("pupil") represents limit configurations when two points $z_{1}, z_{2} \in \mathbb{H}$ collapse into a single point in $\mathbb{H}$, the upper (resp. lower) $\operatorname{lid} C_{1,1}(\mathbb{C}$ ) represents limit configurations of the form $\left(z_{1} \in \mathbb{R}, z_{2} \in \mathbb{H}\right)\left(\right.$ resp. $\left.\left(z_{1} \in \mathbb{H}, z_{2} \in \mathbb{R}\right)\right)$.

\footnotetext{
${ }^{13}$ We tacitly assume here that the $L_{\infty}$-algebra $\left(X_{c}, \nu_{\bullet}\right)$ is appropriately filtered so that the MC equation makes sense. In our applications below $\nu_{n \geq 3}=0$ so that one has no problems with convergence of the infinite sum.
} 
4.3. Higher dimensional version of $C_{n, m}(\mathbb{H})$ and open-closed homotopy Lie algebras. Let $\mathbb{H}^{d}$ stand for a subspace of $\mathbb{R}^{d}=\left\{x_{1}, \ldots, x_{d-1}, x_{d}\right\}$ such that $x_{d}>0, d \geq 2$. The space $\mathbb{H}^{2}$ is just another notation for the the upper-half-plane $\mathbb{H}$. In a full analogy to the case $\bar{C}_{\bullet, \bullet}\left(\mathbb{H}^{2}\right)$ one defines a compactification $\bar{C} \bullet, \bullet\left(\mathbb{H}^{d}\right)$ for any $d \geq 3$ and observes that the disjoint union,

$$
\bar{C}\left(\mathbb{H}^{d}\right):=\bar{C}_{\bullet}\left(\mathbb{R}^{d}\right) \coprod \bar{C}_{\bullet, \bullet}\left(\mathbb{H}^{d}\right)
$$

has a natural structure of a 2-coloured operad in the category of semialgebraic sets. Representations of the associated dg operad of fundamental chains, $\mathcal{F C h a i n s}\left(\bar{C}\left(\mathbb{H}^{d}\right)\right.$, are called open-closed homotopy Lie algebras or, shortly, OCHLA. One can describe this operad in terms of generators of degree $d(1-n)-(d-1) m$,

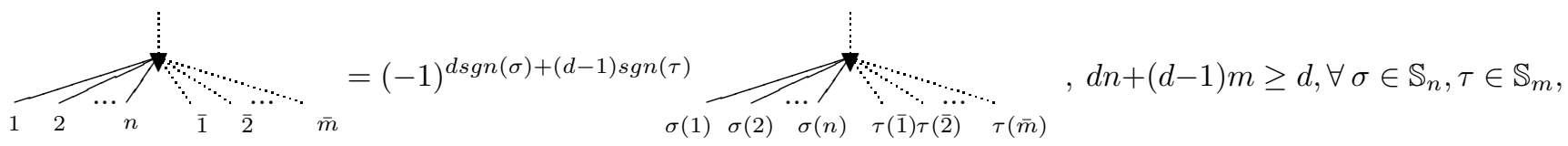

or, equivalently, in terms of its representations in an arbitrary pair, $\left(X_{o}, X_{c}\right)$, of dg vector spaces. We choose here a second more compact option.

As $\mathcal{F C h a i n s}\left(\bar{C}\left(\mathbb{H}^{d}\right)\right.$ is a free operad, its arbitrary representation, $\rho$, in $\left(X_{o}, X_{c}\right)$ is uniquely determined by the values on the generators,

$$
\nu_{n}:=\rho(\overbrace{n, m}=\rho\left(\odot_{n}^{n}\left(X_{c}[d]\right), X_{c}[d+1]\right), \quad n \geq 2,
$$

which satisfy quadratic relations which we explain in the definition of OCHLA. Let us denote the given differential in the dg space $X_{c}$ by $\nu_{1}$ and the one in $X_{o}$ by $\mu_{0,1}$.

A structure of an open-closed homotopy Lie d-algebra in a pair of dg vector spaces $\left(X_{c}, X_{o}\right)$ is, for $d \geq 3$, the data,

(i) a $\mathcal{L}_{\infty}\{d-1\}$-algebra structure, $\nu=\left\{\nu_{n}: \odot^{n}\left(X_{c}[d]\right) \rightarrow X_{c}[d+1]\right\}_{n \geq 1}$, on $X_{c}$, i.e. an ordinary $\mathcal{L}_{\infty}$-structure on $X_{c}[d-1]$;

(ii) a $\mathcal{L}_{\infty}\{d-2\}$-algebra structure, $\mu=\left\{\mu_{0, n}: \odot^{n}\left(X_{o}[d-1]\right) \rightarrow X_{o}[d]\right\}_{n \geq 1}$, on $X_{o}$; the associated MC element, $\mu$, of the Lie algebra $\left(\operatorname{Coder}\left(\odot \bullet\left(X_{o}[d-1]\right)\right),[],\right)$ makes the latter into a $d g$ Lie algebra with the differential $d_{\mu}:=[\mu$,

(iii) a $\mathcal{L}_{\infty}$-morphism, $F$, from the $\mathcal{L}_{\infty}$-algebra $\left(X_{c}[d-1], \nu\right)$ to the $\operatorname{dg}$ Lie algebra $\left(\operatorname{Coder}\left(\odot \bullet\left(X_{o}[d-1]\right)\right)\right.$, [, ], $\left.d_{\mu}\right)$,

$$
F=\left\{F_{n}: \odot^{n}\left(X_{c}[d]\right) \longrightarrow \operatorname{Coder}\left(\odot \bullet\left(X_{o}[d-1]\right)[1]\right)\right\}_{n \geq 1}
$$

such that the composition

$$
\odot^{n}\left(X_{c}[d]\right) \stackrel{F_{n}}{\longrightarrow} \operatorname{Coder}\left(\odot \odot^{\bullet}\left(X_{o}[d-1]\right)\right)[1] \stackrel{\text { proj }}{\longrightarrow} \operatorname{Hom}\left(\odot^{m}\left(X_{o}[d-1]\right), X_{o}[d-1]\right)[1]
$$

coincides precisely with $\mu_{n, m}$ for any $n \geq 1, m \geq 0$.

If $\rho$ is an arbitrary representation of $\mathcal{F C h a i n s}\left(\bar{C}\left(\mathbb{H}^{d}\right)\right.$ for $d \geq 3$ and $\gamma \in X_{c}$ is an arbitrary Maurer-Cartan element,

$$
\sum_{n \geq 0} \frac{1}{n !} \nu_{n}\left(\gamma^{\otimes n}\right)=0, \quad|\gamma|=d
$$

of the associated $\mathcal{L}_{\infty}$-algebra $\left(X_{c}, \nu_{\bullet}\right)$, then the element

$$
F(\gamma):=\sum_{n \geq 1} \frac{\hbar^{n}}{n !} F_{n}\left(\gamma^{\otimes n}\right) \in \operatorname{Coder}\left(\odot \cdot\left(X_{o}^{\hbar}[d-1]\right)[1],\right.
$$

make the topological vector space $X_{o}^{\hbar}:=X_{o}[[\hbar]]$ into a continuous (in general, non-flat) $\mathcal{L}_{\infty}\{d-2\}$-algebra. We show an explicit and non-trivial example of such an open-closed homotopy Lie algebra below in Corollary 9.5.1. below. 


\section{Configuration space models for the 2-coloured operad of $L_{\infty}$-morphisms}

5.1. The complex plane models. The 2 -dimensional Lie group, $G_{(2)}^{\prime}$, of complex translations, $z \rightarrow z+\nu, \nu \in \mathbb{C}$, acts freely on $\operatorname{Conf}_{A}(\mathbb{C})$ (see $\$ \mathbf{4 . 1 . 3}$ for notations used in this subsection),

$$
\begin{array}{ccc}
\operatorname{Conf}_{A}(\mathbb{C}) \times \mathbb{C} & \longrightarrow & \operatorname{Conf}_{A}(\mathbb{C}) \\
\left(p=\left\{z_{i}\right\}_{i \in A}, \nu\right) & \longrightarrow & p+\nu:=\left\{z_{i}+\nu\right\}_{i \in A}
\end{array}
$$

so that the quotient

$$
\mathfrak{C}_{A}(\mathbb{C}):=\operatorname{Conf}_{A}(\mathbb{C}) / G_{(2)}^{\prime}
$$

is a $(2 \# A-2)$-dimensional manifold; as usual, we abbreviate $\mathfrak{C}_{[n]}(\mathbb{C})$ to $\mathfrak{C}_{n}(\mathbb{C})$. There is a diffeomorphism,

$$
\begin{aligned}
& \Psi_{A}: \mathfrak{C}_{A}(\mathbb{C}) \quad \longrightarrow \quad C_{A}^{s t}(\mathbb{C}) \quad \times \quad(0,+\infty) \\
& p \quad \longrightarrow \frac{p-z_{c}(p)}{\left|p-z_{c}(p)\right|} \quad\left|p-z_{c}(p)\right|
\end{aligned}
$$

Note that the configuration $\frac{p-z_{c}(p)}{\left|p-z_{c}(p)\right|}$ is invariant under $\mathbb{R}^{+} \ltimes \mathbb{C}$ and hence gives a well-defined point in $C_{A}^{s t}(\mathbb{C}) \simeq$ $C_{A}(\mathbb{C})$. For any non-empty subset $A \subseteq[n]$ there is a natural map

$$
\begin{array}{cccc}
\pi_{A}: & \mathfrak{C}_{n}(\mathbb{C}) & \longrightarrow & \mathfrak{C}_{A}(\mathbb{C}) \\
& p=\left\{z_{i}\right\}_{i \in[n]} & \longrightarrow & p_{A}:=\left\{z_{i}\right\}_{i \in A}
\end{array}
$$

which forgets all the points labeled by elements of the complement $[n] \backslash A$.

A topological compactification, $\widehat{\mathfrak{C}}_{n}(\mathbb{C})$, of $\mathfrak{C}_{n}(\mathbb{C})$ can be defined as the closure of a composition (cf. [Me2]),

$$
\mathfrak{C}_{n}(\mathbb{C}) \stackrel{\prod \pi_{A}}{\longrightarrow} \prod_{\substack{A \subset[n] \\ \# A \geq 2}} \mathfrak{C}_{A}(\mathbb{C}) \stackrel{\prod^{\Psi_{A}}}{\longrightarrow} \prod_{\substack{A \subseteq[n] \\ \# A \geq 2}} C_{A}^{s t}(\mathbb{C}) \times(0,+\infty) \hookrightarrow \prod_{\substack{A \subset[n] \\ \# A \geq 2}} \widetilde{C}_{A}^{s t}(\mathbb{C}) \times[0,+\infty] .
$$

Thus all the limiting points in this compactification come from configurations when a group or groups of points move too close to each other within each group (as in the case of $\bar{C}_{n}(\mathbb{R})$ ) and/or a group or groups of points are moving too far (with respect to the relative Euclidean distances inside each group) away from each other (cf. §3). The boundary strata in $\widehat{\mathfrak{C}}_{n}(\mathbb{C})$ are given by the limit values 0 or $+\infty$ of the parameters $\left|p_{A}-z_{c}\left(p_{A}\right)\right|, A \subseteq[n]$, and it is an easy (and fully analogous to $\$ \mathbf{3}$ ) exercise to find all the codimension 1 boundary strata,

$$
\partial \widehat{\mathfrak{C}}_{n}(\mathbb{C})=\bigsqcup_{\substack{A \subseteq[n] \\ \# A \geq 2}}\left(\widehat{\mathfrak{C}}_{n-\# A+1}(\mathbb{C}) \times \bar{C}_{\# A}(\mathbb{C})\right) \bigsqcup_{\substack{[n]=B_{1} \leq \ldots, \ldots B_{k} \\ 2 \leq \leq \leq n \\ \# B_{1}, \ldots, \ldots B_{k} \geq 1}}\left(\bar{C}_{k}(\mathbb{C}) \times \widehat{\mathfrak{C}}_{\# B_{1}, 0}(\mathbb{C}) \times \ldots \times \widehat{\mathfrak{C}}_{\# B_{k}, 0}(\mathbb{C})\right)
$$

where the first summation runs over all possible subsets, $A$, of $[n]$ with cardinality at least two, and the second summation runs over all possible decompositions of $[n]$ into (at least two) disjoint non-empty subsets $B_{1}, \ldots, B_{k}$. Geometrically, a stratum in the first group of summands corresponds to $A$-labeled elements of the set $\left\{z_{1}, \ldots, z_{n}\right\}$ moving close to each other, while a stratum in the second group of summands corresponds to $k$ clusters of points (labeled, respectively, by disjoint ordered subsets $B_{1}, \ldots B_{k}$ of $[n]$ ) moving far from each other while keeping relative distances within each group $B_{i}$ finite.

Note that the faces of the type $C \cdot(\mathbb{C})$ appear in the natural stratification of $\widehat{\mathfrak{C}}_{n}(\mathbb{C})$ in two ways - as the strata describing collapsing points and as the strata controlling groups of points at "infinity" — and they never intersect in $\widehat{\mathfrak{C}}_{n}(\mathbb{C})$ (cf. $\oiint_{3}$ ). For that reason we have to assign to these two groups of faces different colours and represent collapsing $\bar{C}_{n}(\mathbb{C})$-stratum by, say, white corolla with straight legs as in (14), the $\bar{C}_{n}(\mathbb{R})$-stratum at "infinity" by, say, a version of (14) with "broken" legs, of degree $2-2 n$.

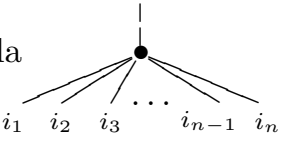


5.1.1. Proposition (cf. $[\mathrm{Me} 2]$ ). The face complex of the disjoint union

$$
\widehat{\mathfrak{C}}(\mathbb{C}):=\bar{C} \bullet(\mathbb{C}) \sqcup \widehat{\mathfrak{C}}_{\bullet}(\mathbb{C}) \sqcup \bar{C} \cdot(\mathbb{C})
$$

has naturally a structure of a $d g$ free non-unital 2-coloured operad of transformation type,

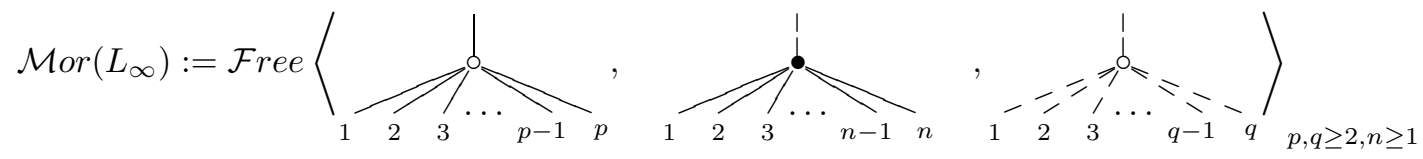

equipped with a differential which is given on white corollas of both colours by formula (15) and on black corollas by the following formula

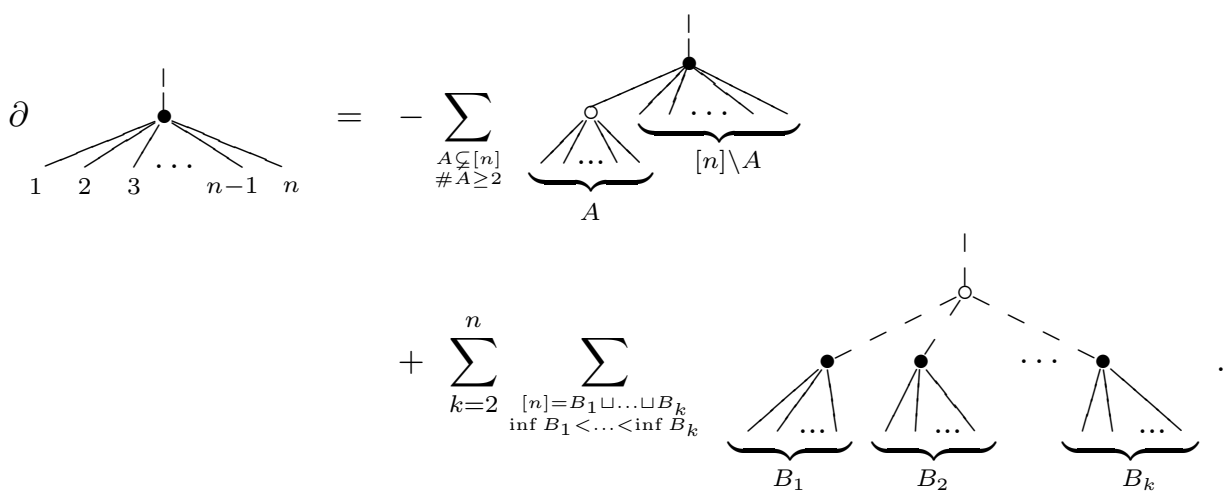

Representations of this operad in a pair of $d g$ vector spaces, $V_{\text {in }}$ and $V_{\text {out }}$, is the same as a triple, $\left(\mu_{\text {in }}, \mu_{\text {out }}, F\right)$, consisting of $\mathcal{L}_{\infty}\{1\}$ structures, $\mu_{\text {in }}$ on $V_{\text {in }}$ and $\mu_{\text {out }}$ on $V_{\text {out }}$, and of a $\mathcal{L}_{\infty}\{1\}$ morphism, $F:\left(V_{\text {in }}, \mu_{\text {in }}\right) \rightarrow$ $\left(V_{\text {out }}, \mu_{\text {out }}\right)$, between them.

5.1.2. Example. As $C_{2}^{s t}(\mathbb{C})=\widetilde{C}_{2}(\mathbb{C})=S^{1}$, the space $\widehat{\mathfrak{C}}_{2}(\mathbb{C})$ is the closure of the embedding

$$
\begin{array}{llllll}
\mathfrak{C}_{2}(\mathbb{C}) & \longrightarrow & S^{1} & \times & (0,+\infty) \\
\left(z_{1}, z_{2}\right) & \longrightarrow & \operatorname{Arg}\left(z_{1}-z_{2}\right) & & & \left|z_{1}-z_{2}\right|
\end{array}
$$

and hence can be identified with the closed cylinder

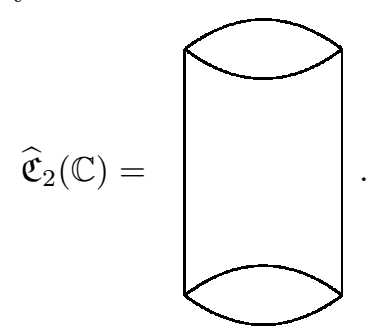

5.1.3. Smooth (or semialgebraic) structure. The embedding formula (19) makes $\widehat{\mathfrak{C}}(\mathbb{C})$ into an operad in the category of semialgebraic manifolds. We can make it also into an operad in the category of smooth manifolds with corners using metric trees in almost exactly the same way as in $\mathbf{2 . 2}$.

5.1.4. A second complex space model for $\mathcal{M o r}\left(L_{\infty}\right)$. In a full analogy to 3.2 we can introduce on $\widehat{\mathfrak{C}}(\mathbb{C})$ a different smooth structure using a different compactification formula. For a pair of subspaces $B \subsetneq A \subseteq[n]$ we consider

$$
\begin{array}{rllc}
\pi_{A, B}: \mathfrak{C}_{n}(\mathbb{C}) & \longrightarrow & C_{B}^{s t}(\mathbb{C}) \\
p & \longrightarrow \frac{p_{B}-z_{c}\left(p_{B}\right)}{\left|p_{B}-z_{c}\left(p_{B}\right)\right|} & & \left|p_{A}-z_{c}\left(p_{B}\right)\right| \cdot\left|p_{B}-z_{c}\left(p_{B}\right)\right|
\end{array}
$$

and then define a topological compactification $\widehat{\mathfrak{C}}_{\bullet}(\mathbb{C})$ as the closure of the following composition of embeddings,

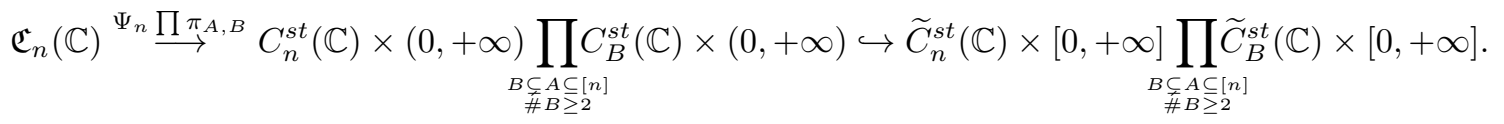

The boundary strata in $\widehat{\mathfrak{C}}_{n}(\mathbb{C})$ are given by the limit values 0 or $+\infty$ of the parameters $\left|p_{[n]}-z_{c}\left(p_{[n]}\right)\right|$ and $\left|p_{A}-z_{c}\left(p_{B}\right)\right| \cdot\left|p_{B}-z_{c}\left(p_{B}\right)\right|$, and the combinatorics of its face complex is again described by Proposition 5.1.1. 
However, this compactification has a different geometric meaning from the one given by the embedding formula (19); we refer to $\$ 3.2$ for detailed discussion of the 1-dimensional version of this phenomenon. Smooth structure on this compactification can be introduced in a complete analogy to $\$ \mathbf{3 . 2}$.

5.2. Upper half space models for $\mathcal{M o r}\left(L_{\infty}\right)$ Me2]. Let $\operatorname{Conf}_{A, 0}(\mathbb{H})$ stand for the space of injections, $A \hookrightarrow \mathbb{H}$, of a finite set $A$ into the upper half-plane, and $\widetilde{\operatorname{Conf}} A, 0(\mathbb{H})$ for the space of maps, $A \rightarrow \mathbb{H}$. In this section we remind a compactification, $\widehat{C}_{A, 0}(\mathbb{H})$, of Kontsevich's configuration space,

$$
C_{A, 0}(\mathbb{H})=\operatorname{Conf}_{A, 0}(\mathbb{H}) / G_{(2)}, \quad \# A \geq 1,
$$

which is different from Kontsevich's one and which gives us an upper half space model for the 2-coloured operad of homotopy morphisms of $\mathcal{L}_{\infty}$-algebras. It is worth noting that the group $G_{(2)}^{\prime}$ used earlier to construct a complex space model, $\widehat{\mathfrak{C}}_{\bullet}(\mathbb{C})$, for $\operatorname{Mor}\left(\mathcal{L}_{\infty}\right)$ is obtained from the group $G_{(3)}=\mathbb{R}^{+} \ltimes \mathbb{C}$ (which was used to construct a configuration space model for the operad of $\mathcal{L}_{\infty}$-algebras) by taking away dilations $\mathbb{R}^{+}$, while this time we use points in the upper half plane together with the group $G_{(2)}$ obtained from $G_{(3)}$ by taking away the semigroup of vertical translations $\mathbb{R}^{+}$.

Define a section,

$$
\begin{array}{ccc}
s: & C_{A, 0}(\mathbb{H}) & \operatorname{Conf}_{A, 0}(\mathbb{H}) \\
p=\left\{z_{i}=x_{i}+\mathfrak{i} y_{i} \in \mathbb{H}\right\}_{i \in A} & \longrightarrow & p^{s t}:=\frac{p-x_{c}(p)}{\inf _{i \in A} y_{i}} .
\end{array}
$$

where $x_{c}(p):=\sum_{i=1}^{\# A} \frac{1}{\# A} x_{i}$, and set $C_{A, 0}^{s t}(\mathbb{H}):=\operatorname{Im} s$. Note that every point in the configuration $p^{s t}$ lies in the subspace $\Im z \geq 1 \subset \mathbb{H}$ and at least one point lies on the line $\Im z=1$. Thus

$$
C_{A, 0}^{s t}(\mathbb{H})=\left\{p=\left\{z_{i}\right\}_{i \in A} \in \operatorname{Conf}_{A, 0}(\mathbb{H}) \mid x_{c}(p)=0, \inf _{i \in A} y_{i}=1\right\}
$$

It is an elementary exercise to check that the subspace $C_{A}^{s t}(\mathbb{H}) \subset C_{A, 0}^{s t}(\mathbb{H})$ consisting of elements $p^{s t}$ with

$$
\left|p^{s t}-\mathfrak{i}\right|=1
$$

gives a global section of the surjective forgetful map $\operatorname{Conf}_{A, 0}(\mathbb{H}) \rightarrow C_{A}(\mathbb{C})$ and hence is homeomorphic to $C_{A}(\mathbb{C})$. Note that both spaces $C_{n, 0}^{s t}(\mathbb{H})$ and $C_{n}^{s t}(\mathbb{H})$ have natural structures of smooth manifolds with corners (and also of semialgebraic sets); for example,

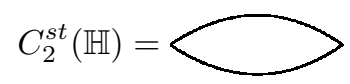

rather than an ordinary smooth circle $S^{1}$. Thus

$$
C_{A}^{s t}(\mathbb{H})=\left\{p=\left\{z_{i}\right\}_{i \in A} \in \operatorname{Conf}_{A, 0}(\mathbb{H})\left|x_{c}(p)=0, \inf _{i \in A} y_{i}=1,\right| p-\mathfrak{i} \mid=1\right\} .
$$

We also define

$$
\widetilde{C}_{A}^{s t}(\mathbb{H})=\left\{p=\left\{z_{i}\right\}_{i \in A} \in \widetilde{\operatorname{Conf}}_{A, 0}(\mathbb{H})\left|x_{c}(p)=0, \inf _{i \in A} y_{i}=1,\right| p-\mathfrak{i} \mid=1\right\} .
$$

which is a compact manifold with corners. There is a homeomorphism,

$$
\begin{array}{ccccc}
\Xi_{n}: \quad C_{n, 0}(\mathbb{H}) & \longrightarrow & C_{n}^{s t}(\mathbb{H}) \simeq C_{n}(\mathbb{C}) & \times & (0,+\infty) \\
p & \longrightarrow & \frac{p^{s t}-\mathfrak{i}}{\left|p^{s t}-\mathfrak{i}\right|}+\mathfrak{i} & \times & \left|p^{s t}-\mathfrak{i}\right| .
\end{array}
$$

Let, for a subset $A \subset[n]$,

$$
\begin{array}{cccc}
\pi_{A}: & C_{n, 0}(\mathbb{H}) & \longrightarrow & C_{A, 0}(\mathbb{H}) \\
& p=\left\{z_{i}\right\}_{i \in[n]} & \longrightarrow & p_{A}=\left\{z_{i}\right\}_{i \in A}
\end{array}
$$

stand for the natural forgetful map. For a pair of subspaces $B \subsetneq A \subseteq[n]$ we consider a map

$$
\begin{array}{ccccc}
\Xi_{A, B}: & C_{n, 0}(\mathbb{H}) & \longrightarrow & C_{B}^{s t}(\mathbb{H}) \\
p & \longrightarrow & \frac{p_{B}-z_{\min }\left(p_{B}\right)}{\left|p_{B}-z_{\min }\left(p_{B}\right)\right|}+\mathfrak{i} \\
& & & & \left\|p_{A, B}\right\|:=\frac{\left|p_{B}-z_{\min }\left(p_{B}\right)\right|}{y_{\min }\left(p_{A}\right)} .
\end{array}
$$


Depending on application needs, a topological compactification, $\widehat{C}_{n, 0}(\mathbb{H})$, of $C_{n, 0}(\mathbb{H})$ can be defined either as the closure of a composition (cf. (5) and (19)),

$$
C_{n, 0}(\mathbb{H}) \stackrel{\prod_{A}}{\longrightarrow} \prod_{\substack{A \subseteq[n] \\ A \neq \emptyset}} C_{A, 0}(\mathbb{H}) \stackrel{\prod^{\Xi_{A}}}{\longrightarrow} \prod_{\substack{A \subseteq[n] \\ A \neq \emptyset}} C_{A}^{s t}(\mathbb{H}) \times(0,+\infty) \longrightarrow \prod_{\substack{A \subseteq[n] \\ A \neq \emptyset}} \widetilde{C}_{A}^{s t}(\mathbb{H}) \times[0,+\infty] .
$$

or as the closure on the following embedding,

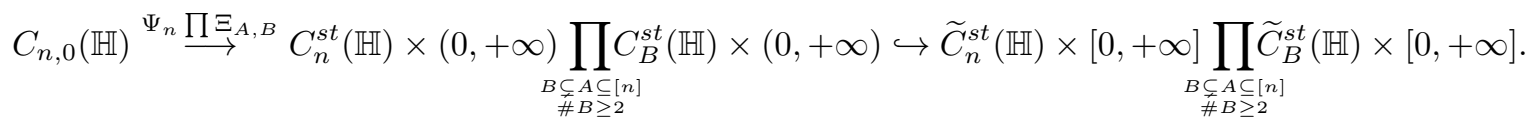

The boundary strata in both cases are given by the limit values 0 or $+\infty$ of the parameters $\left|p^{s t}-\mathfrak{i}\right|$ and $\left|p_{A}^{s t}-\mathfrak{i}\right|$ (respectively, $\left|p^{s t}-\mathfrak{i}\right|$ and $\left.\| p_{A, B}||\right)$. It is not hard to see that the combinatorics of the face complex of $\widehat{C}_{\bullet, 0}(\mathbb{H})$ is the same as in the case of $\widehat{\mathfrak{C}}_{\bullet}(\mathbb{C})$ so that $\widehat{C}_{\bullet, 0}(\mathbb{H})$ gives us a configuration space model for the 2-coloured operad of $\mathcal{L}_{\infty^{-}}$ algebras and their homotopy morphisms $\mathrm{Me} 2$. However, the geometric meaning and the natural smooth structure on $\widehat{\mathfrak{C}}_{.}(\mathbb{H})$ are not equivalent to the ones studied above. Note that contrary to the Kontsevich compactification, $\bar{C}_{n, 0}(\mathbb{H})$, of $C_{n, 0}(\mathbb{H})$ limit configurations in $\widehat{C}_{n, 0}(\mathbb{H})$ never approach the real line in $\overline{\mathbb{H}}$. From now on we use the symbol $\widehat{C}_{n, 0}(\mathbb{H})$ to denote the closure of the embedding (27).

5.2.1. Smooth atlas on $\widehat{C}_{\bullet, 0}(\mathbb{H})$. An atlas on the topological operad

$$
\widehat{C}(\mathbb{H}):=\bar{C} \cdot(\mathbb{C}) \sqcup \widehat{C}_{\bullet}, 0(\mathbb{H}) \sqcup \bar{C} \bullet(\mathbb{C})
$$

can be constructed with the help of exactly the same kind of metric trees as the ones used in Section $\mathbf{3 . 2}$ (see also (3.4):

- the spaces of standard positions associated with white $n$-corollas of both colours are set to be $C_{n}^{\text {st }}(\mathbb{H})$, and the space of standard positions associated with the black $n$-corolla is $C_{n, 0}^{s t}(\mathbb{H})$;

- the rescaling operation is defined on $C_{n}^{s t}(\mathbb{H})$ and $C_{n, 0}^{s t}(\mathbb{H})$ by the map, $z \rightarrow \lambda(z-i)+i$;

- for a point $z_{0}=x_{0}+\mathfrak{i} y_{0}$ the associated translation map $T_{z_{0}}: \operatorname{Conf}_{n, 0}(\mathbb{H}) \rightarrow \operatorname{Conf}_{n, 0}(\mathbb{H})$ is defined to be $p \rightarrow p+z_{0}$.

This atlas makes $\widehat{C}(\mathbb{H})$ into an operad in the category of smooth manifolds with corners. For example, the space $\widehat{C}_{2,0}(\mathbb{H})$ is the closure of an embedding,

$$
C_{2,0}(\mathbb{H}) \quad \longrightarrow \quad C_{2}^{s t}(\mathbb{H}) \times[0,+\infty]
$$

and hence is diffeomorphic to the following manifold with corners

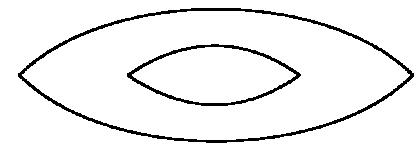

whose inner topological circle represents the boundary component, $C_{1,0}(\mathbb{H}) \times C_{2}^{s t}(\mathbb{H})$, describing two point moving very close to each other while the outer topological circle describes the boundary component describing two points moving very far - in the Euclidean or Poincaré metric — from each other.

5.2.2. Remark. We can define a slightly different smooth structure on $\widehat{C} \bullet(\mathbb{H})$ by associating $C_{n}^{s t}(C)$ to white corollas with solid legs and $C_{n}^{s t}(\mathbb{H})$ to white corollas with broken legs. Then the rescaling operation on $C_{n}^{s t}(\mathbb{C})$ has to be defined as an ordinary dilation, $z \rightarrow \lambda z$. In this smooth structure on $\widehat{\mathfrak{C}}$. $(\mathbb{H})$ the space $\widehat{C}_{2,0}(\mathbb{H})$ is precisely the "Kontsevich eye",

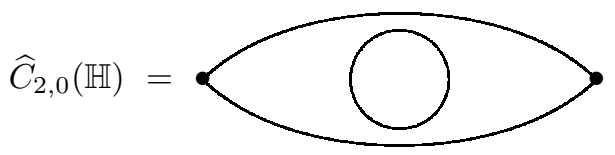

There is no big difference between these two smooth structures as in both cases the Kontsevich propagator $\omega_{K}=$ $d A r g \frac{z_{1}-z_{2}}{\bar{z}_{1}-z_{2}}$ is a smooth differential 1-form on $\widehat{C}_{2,0}(\mathbb{H})$. 
5.2.3. Higher dimensional versions. In a full analogy one can define a compactification, $\widehat{C}_{n, 0}\left(\mathbb{H}^{k}\right)$, of the orbit space $C_{n, 0}\left(\mathbb{H}^{k}\right)$ for any $k \geq 2$ and check that the face complex of the disjoint union

$$
\widehat{C}\left(\mathbb{H}^{d}\right):=\bar{C}_{\bullet}\left(\mathbb{R}^{d}\right) \sqcup \widehat{C}_{\bullet}, 0\left(\mathbb{H}^{d}\right) \sqcup \bar{C}_{\bullet}\left(\mathbb{R}^{d}\right)
$$

is a dg free 2-coloured operad of morphisms of $\mathcal{L}_{\infty}\{k\}$-algebras. In fact, we can talk about this family of operads in the range $k \geq 1$ : the case $k=1$ gives us the 2-coloured operad of $\mathcal{A}_{\infty}$-morphisms, and all the other cases give us the (degree shifted) 2-coloured operad of $\mathcal{L}_{\infty}$-morphisms; the topological reason for this phenomenon is clear.

\section{Configuration space model for the 4-coloured operad of OCHA morphsisms}

6.1. New compactified configuration spaces $\widehat{\mathfrak{C}}_{n, m}(\mathbb{H})$. Let us define, for $2 n+m \geq 1$,

$$
\mathfrak{C}_{n, m}(\mathbb{H}):=\operatorname{Conf}_{n, m}(\mathbb{H}) / G_{(1)},
$$

where the Lie group $G_{(1)}=\mathbb{R}$ acts on $\overline{\mathbb{H}}$ by translations,

$$
G_{(1)}=\{z \rightarrow z+\nu \mid \nu \in \mathbb{R}\} .
$$

This is a $(2 n+m-1)$-dimensional naturally oriented manifold which is isomorphic to $C_{n, m}(\mathbb{H}) \times \mathbb{R}^{+}$via the following map,

$$
\begin{aligned}
& \Phi_{n, m}: \quad \mathfrak{C}_{n, m}(\mathbb{H}) \quad \longrightarrow \quad C_{n, m}(\mathbb{H}) \times \mathbb{R}^{+} \\
& p \quad \longrightarrow \frac{p-x_{c}(p)}{\left|p-x_{c}(p)\right|} \times\left|p-x_{c}(p)\right| .
\end{aligned}
$$

Note that the fraction $\left(p-x_{c}(p)\right) /\left|p-x_{c}(p)\right|$ is $G_{(2)}$-invariant and hence gives a well-defined element in $C_{n, m}(\mathbb{H})$. Recall that

$$
C_{n, m}^{s t}(\mathbb{H})=\left\{p \in \operatorname{Conf}_{n, m}(\mathbb{H})\left|x_{c}(p)=0, \quad\right| p \mid=1\right\}
$$

gives a section of the natural projection $\operatorname{Conf}_{n, m}(\mathbb{H}) \rightarrow C_{n, m}(\mathbb{H})$. We also consider

$$
\widetilde{C}_{n, m}^{s t}(\mathbb{H})=\left\{p \in \widetilde{\operatorname{Conf}}_{n, m}(\mathbb{H})\left|x_{c}(p)=0, \quad\right| p \mid=1\right\}
$$

which is a compact manifold with boundary.

For a pair of subsets $A \subset[n]$ and $B \subset[m]$, let

$$
\begin{array}{cccc}
\pi_{A, B}: & \mathfrak{C}_{n, m}(\mathbb{H}) & \longrightarrow & \mathfrak{C}_{A, B}(\mathbb{H}) \\
& p=\left\{z_{i}, x_{j}\right\}_{i \in[n], j \in[m]} & \longrightarrow & p_{A, B}:=\left\{z_{i}, x_{j}\right\}_{i \in A, j \in B}
\end{array}
$$

be the forgetful map. We also consider a map

$$
\begin{array}{rcccc}
\Xi_{A, 0}: \quad \mathfrak{C}_{A, B}(\mathbb{H}) & \stackrel{\pi_{A \sqcup \varphi}}{\longrightarrow} \mathfrak{C}_{A, 0}(\mathbb{H}) & \longrightarrow & C_{A}(\mathbb{C}) \times(0+\infty) \\
p=\left\{z_{i}, x_{j}\right\}_{i \in[A], j \in[B]} & \longrightarrow p_{A}=\left\{z_{i}\right\}_{i \in A} & \longrightarrow & \left(\frac{p_{A}-z_{c}\left(p_{A}\right)}{\left|p_{A}-z_{c}\left(p_{A}\right)\right|},\left|p_{A}-z_{c}\left(p_{A}\right)\right|\right)
\end{array}
$$

where $z_{c}(p):=\frac{1}{\# A} \sum_{i \in A} z_{i}$. Note that the fraction $\left(p_{A}-z_{c}\left(p_{A}\right)\right) /\left|p_{A}-z_{c}\left(p_{A}\right)\right|$ is $G_{(3)}$-invariant and hence gives a well-defined element in $C_{A}^{s t}(\mathbb{C}) \simeq C_{A}(\mathbb{C})$.

6.1.1. Definition. A topological compactification, $\widehat{\mathfrak{C}}_{n, m}(\mathbb{H})$, of $\mathfrak{C}_{n, m}(\mathbb{H})$ can be defined as the closure of a composition (cf. (5) and (19))

$$
\begin{aligned}
\mathfrak{C}_{n, m}(\mathbb{H}) \stackrel{\prod \pi_{A, B}}{\longrightarrow} \prod_{\substack{A \subseteq[n], B \subset[m] \\
\# 2 A+\# B \geq 1}} \mathfrak{C}_{A, B}(\mathbb{H}) \stackrel{\prod \Phi_{A, B} \times \prod \Xi_{A, 0}}{\longrightarrow} & \prod_{\substack{A \subseteq[n], B \subseteq[m] \\
\# 2 A+\# \bar{B} \geq 1}}\left(C_{A, B}^{s t}(\mathbb{H}) \times \mathbb{R}^{+}\right) \times \prod_{\substack{A \subset[n] \\
\# \bar{A} \geq 2}}\left(C_{A}^{s t}(\mathbb{C}) \times \mathbb{R}^{+}\right) \\
& \prod_{\substack{A \subseteq[n], B \subseteq[m] \\
\# 2 A+\# B \geq 1}}\left(\widetilde{C}_{A, B}^{s t}(\mathbb{H}) \times \overline{\mathbb{R}^{+}}\right) \times \prod_{\substack{A \subseteq[n] \\
\# A \geq 2}}\left(\widetilde{C}_{A}^{s t}(\mathbb{C}) \times \overline{\mathbb{R}^{+}}\right)
\end{aligned}
$$

The boundary strata in both definitions are given by the limit values, 0 or $+\infty$, of the parameters, $\left\{\left|p_{A, B}-x_{c}\left(p_{A, B}\right)\right|, \quad\left|p_{A}-z_{c}\left(p_{A}\right)\right|\right\}_{A \subseteq[n], B \subseteq[m]}$ so that all the limiting points in this compactification come from configurations when a group or groups of points move too close to each other within each group and/or a group or groups of points are moving too far (with respect to the relative Euclidean distances inside each group) away 
from each other. It is obvious that the family $\widehat{\mathfrak{C}}_{0, \bullet}(\mathbb{H})$ is precisely the family of compactified configuration spaces defined in $₫ 3$ and hence describes the 2-coloured operad of $A_{\infty}$-morphisms. We claim that $\widehat{\mathfrak{C}}_{\bullet} . \bullet(\mathbb{H})$ unifies this 2-coloured operad with the 2-coloured operad $\widehat{C}_{\bullet, 0}(\mathbb{H})$ describing $\mathcal{M o r}\left(L_{\infty}\right)$ into a 4-coloured operad with an expected meaning - it gives a geometric model for the operad, $\operatorname{Mor}\left(\mathcal{O C}_{\infty}\right)$, of homotopy morphisms of OCHA algebras introduced in the analytic form in KaSt. Let us first give a precise definition of $\mathcal{M o r}\left(\mathcal{O C}_{\infty}\right)$ and then prove the claim.

6.1.2. Morphisms of OCHA algebras KaSt. The 4-coloured operad, $\operatorname{Mor}\left(\mathcal{O C}_{\infty}\right)$, is a dg free operad generated by two copies,

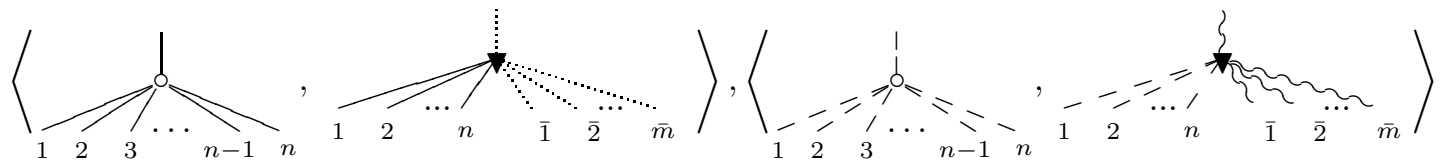

of the operad $\mathcal{O C}_{\infty}$, one copy, \langle $\mathbb{S}_{N}$-modules, $N \geq 1$

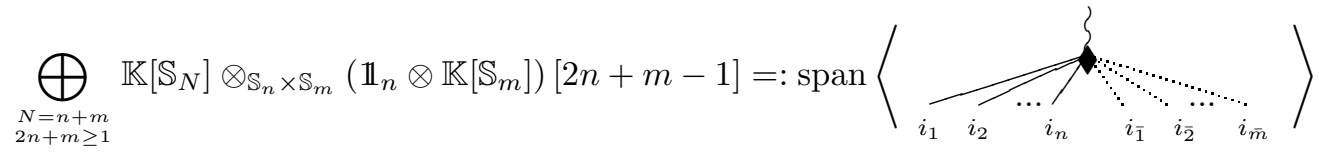

where $\mathbb{1}_{n}$ stands for the trivial representation of $\mathbb{S}_{n}$ (implying that the solid input legs of the - -corolla are "symmetric" as in (17)). The differential $\partial$ is given on o-, $\mathbf{\nabla}$ - and $\bullet$-corollas by formulae (15), (18), and, respectively, $(22)$, and on -corollas by the following formula,

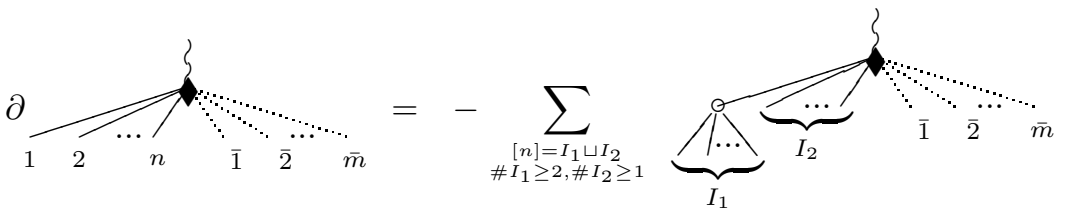

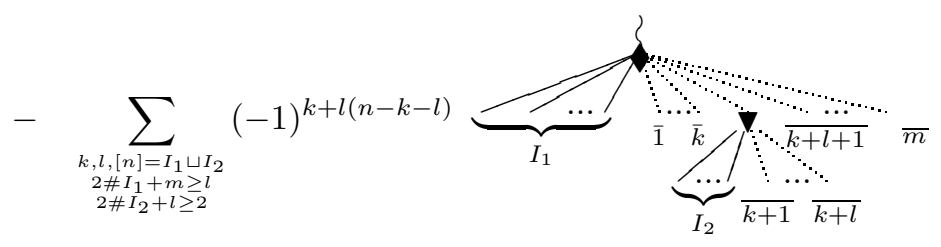

$$
\begin{aligned}
& +\sum_{\substack{k, l \geq 0 \\
2 k+l \geq 2}} \sum_{\substack{[n]=I_{1} \sqcup \ldots, J_{l} \\
m=m_{1}+\ldots+m_{l}}}(-1)^{\sum_{i=1}^{l}(l-i)\left(m_{i}-1\right)} \underbrace{\ldots}_{I_{1}} \underbrace{\ldots}_{I_{k}} \underbrace{\ldots}_{J_{1}}
\end{aligned}
$$

Representations of this 4-coloured operad in a 4-tuple of dg vector spaces, $V_{c}^{\text {in }}, V_{o}^{\text {in }}, V_{c}^{\text {out }}$ and $V_{o}^{\text {out }}$, is the same as a pair, $\left(V_{c}^{\text {in }}, V_{0}^{\text {in }}\right)$ and $\left(V_{c}^{\text {out }}, V_{0}^{\text {out }}\right)$, of homotopy open-closed algebras, and a homotopy morphism, $F:\left(V_{c}^{\text {in }}, V_{0}^{\text {in }}\right) \rightarrow$ $\left(V_{c}^{\text {out }}, V_{o}^{\text {out }}\right)$, between them as defined in KaSt].

6.2. Theorem on the face complex of $\widehat{\mathfrak{C}}_{\bullet, . \bullet}$. The face complex of the disjoint union,

$$
\widehat{\mathfrak{C}}(\mathbb{H}):=\underbrace{\bar{C} \bullet(\mathbb{C}) \bigsqcup \bar{C}_{\bullet, \bullet}(\mathbb{H})}_{\text {in }} \sqcup \widehat{\mathfrak{C}}_{\bullet, \bullet}(\mathbb{H}) \bigsqcup \widehat{\mathfrak{C}}_{\bullet}(\mathbb{C}) \bigsqcup \underbrace{\bar{C}_{\bullet}(\mathbb{C}) \bigsqcup \bar{C}_{\bullet, \bullet}(\mathbb{H})}_{\text {out }}
$$


has a natural structure of a $d g$ free 4-coloured operad canonically isomorphic to the operad $\mathcal{M o r}\left(\mathcal{O C}_{\infty}\right)$. The canonical isomorphism is given by the following identifications,

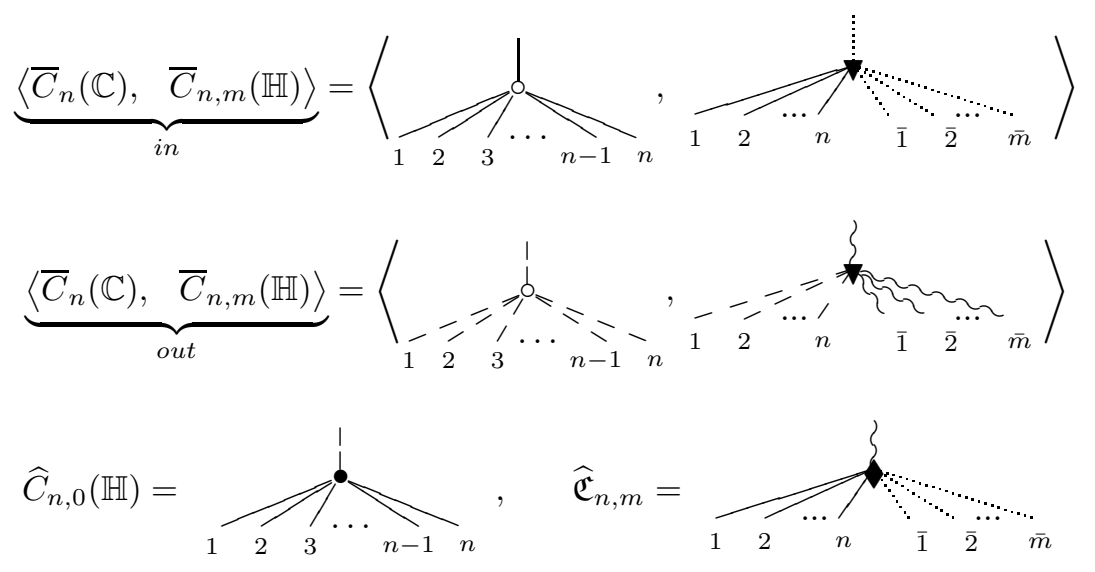

Proof. The codimension 1 boundary strata in $\widehat{\mathfrak{C}}_{n, m}(\mathbb{H})$ are given by the limit values, 0 and $+\infty$, of the parameters

$$
\left\{|| p_{A, B}\left\|:=\left|p_{A, B}-x_{c}\left(p_{A, B}\right)\right|, \quad\right\| p_{A} \|_{0}:=\left|p_{A}-z_{c}\left(p_{A}\right)\right|\right\}_{A \subset[n], B \subseteq[m]} .
$$

(i) The limit configurations, $p \in \widehat{\mathfrak{C}}_{n, m}(\mathbb{H})$, filling in the boundary stratum

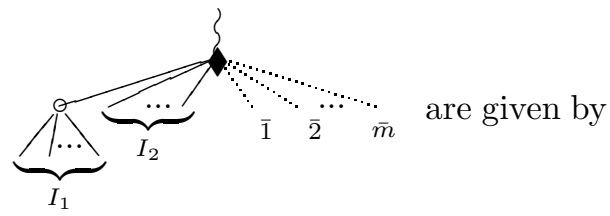

$\left\|p_{I_{1}}\right\|_{0}=0,\|p\|$ is a finite number.

(ii) The limit configurations, $p \in \widehat{\mathfrak{C}}_{n, m}(\mathbb{H})$, filling in the boundary stratum

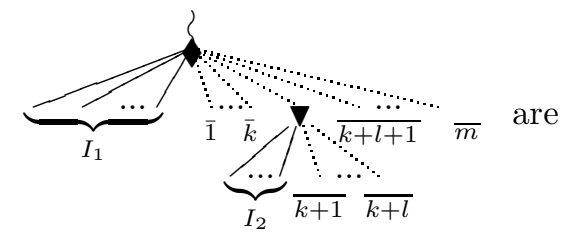

given by the equation $\left\|p_{I_{2},\{\overline{k+1} \ldots, \overline{k+l}\}}\right\|=0,\|p\|$ is a finite number.

(iii) The limit configurations, $p \in \widehat{\mathfrak{C}}_{n, m}(\mathbb{H})$, filling in the boundary stratum

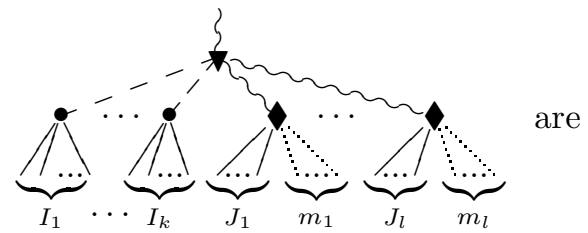

characterized by the following data: $\left\|p_{[n],[m]}\right\|=+\infty,\left\|p_{I_{i}}\right\|_{0}$ is finite for all $i \in[k],\left\|p_{J_{i}, m_{j}}\right\|$ is finite for all $j \in[l]$, and the image of $p$ under the projection $\widehat{\mathfrak{C}}_{n, m}(\mathbb{H}) \rightarrow \widetilde{C}_{n, m}^{s t}(\mathbb{H})$ consists of $k$ different points in the upper-half-plane and $l$ different points on the real line. This is the case when $k$ groups of points in $\mathbb{H}$ parameterized by sets $I_{1}, \ldots, I_{k}$, and $l$-groups of points in $\overline{\mathbb{H}}$ parameterized by sets $J_{1} \sqcup m_{1}, \ldots, J_{l} \sqcup m_{l}$ are moving far away from each other in such a way that their sizes (measured by the parameters $\left\|p_{I_{i}}\right\|_{0}$ and $\left.\left\|p_{J_{i}, m_{j}}\right\|\right)$ stay finite.

Finally, it is an elementary calculation to check that all the boundary strata defined above have codimension 1, and these are the only boundary strata satisfying this condition.

Let us illustrate the above theorem with several explicit examples.

6.2.1. The case $\widehat{\mathfrak{C}}_{0, \bullet}(\mathbb{H})$. The configuration spaces $\left\{\widehat{\mathfrak{C}}_{0, m}(\mathbb{H})\right\}_{m \geq 1}$ are precisely Stasheff's multiplihedra (see $₫ 3$ ), and the formula (29) indeed reduces in this case to (7). 
6.2.2. The case $\widehat{\mathfrak{C}}_{1,0}(\mathbb{H})$. In the case $n=1, m=0$ formula (29) gives,

$$
\partial\left\{=-\frac{\xi}{\xi}+\frac{\xi}{i}\right.
$$

On the other hand, $\mathfrak{C}_{1,0}(\mathbb{H})$ is isomorphic to $(0,+\infty)$, the $y$-axis in $\mathbb{H}$, and $\widehat{\mathfrak{C}}_{1,0}(\mathbb{H})$ is the closure of the embedding $(0,+\infty) \hookrightarrow[0,+\infty]$. Hence $\widehat{\mathfrak{C}}_{1,0}(\mathbb{H})$ is the closed interval $[0,+\infty]$ with the boundary operator $\partial$ coinciding precisely with the above formula if we use the identifications of configuration spaces with graphs given in Theorem 30

6.2.3. The case $\widehat{\mathfrak{C}}_{1,1}(\mathbb{H})$. In the case $n=1, m=1$ formula (29) gives,

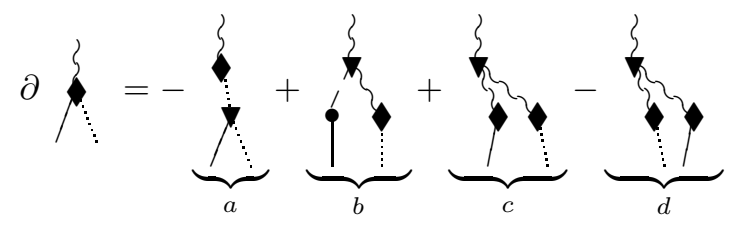

On the other hand, the compactifying embedding takes the following explicit form

$$
\begin{gathered}
\mathfrak{C}_{1,1}(\mathbb{H}) \longrightarrow\left(\widetilde{C}_{1,1} \times[0,+\infty]\right) \\
p=(\underbrace{x_{1}+\mathfrak{i} y_{1}}_{p_{1}}, x_{2}) \longrightarrow\left(\frac{p-x_{c}(p)}{\|p\|}=\frac{\frac{1}{2}\left(x_{1}-x_{2}\right)+\mathfrak{i} y_{1},-\frac{1}{2}\left(x_{1}-x_{2}\right)}{\sqrt{y_{1}^{2}+\frac{1}{2}\left(x_{1}-x_{2}\right)^{2}}},\|p\|\right) \\
\times\left(\widetilde{C}_{1,0} \times[0,+\infty]\right) \\
\left(i, y_{1}\right)
\end{gathered}
$$

In this approach

- the boundary stratum $a=\bar{C}_{1,1}(\mathbb{H})$ is given by the limit configurations with $\|p\| \rightarrow 0$;

- the boundary stratum $b=\bar{C}_{1,1}(\mathbb{H})$ is given by the limit configurations with $\left\|y_{1}\right\| \rightarrow+\infty$,

- the boundary stratum $c$ and $d$ are given by the limit configurations with $\|p\| \rightarrow+\infty$ and $y_{1}$ finite, i.e. as the limit configurations $\left(\frac{\lambda}{2}\left(x_{1}-x_{2}\right)+\mathfrak{i} y_{1},-\frac{\lambda}{2}\left(x_{1}-x_{2}\right)\right)$ when $|\lambda| \rightarrow+\infty$; the case $\lambda \rightarrow+\infty$ corresponds to $c$ and the case $\lambda \rightarrow-\infty$ to $d$.

Each term on the r.h.s. of (31) stands therefore for a closed interval. We finally get the following picture,

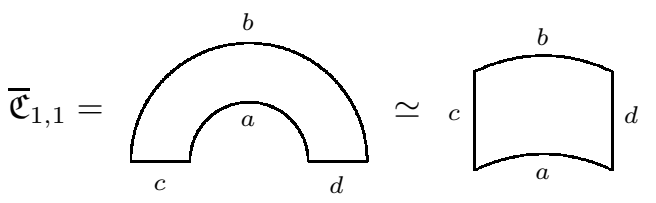


6.2.4. The case $\widehat{\mathfrak{C}}_{2,0}(\mathbb{H})$. In the case $n=2, m=0$ formula (29) gives (see picture (32) below which visualizes each summand's contribution into the boundary of $\left.\widehat{\mathfrak{C}}_{2,0}(\mathbb{H})\right)$,

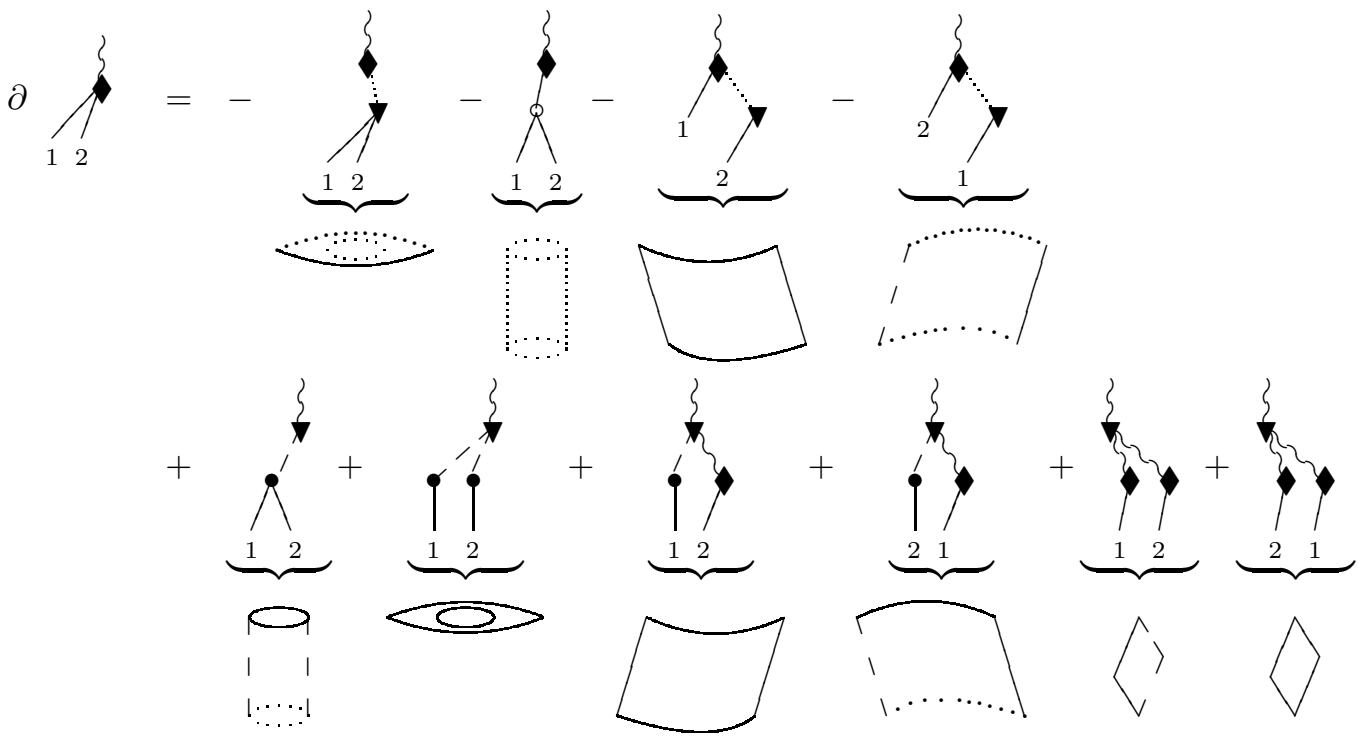

On the other hand the compactification formula (28) takes in this case the form,

$$
\begin{gathered}
\mathfrak{C}_{2,0}(\mathbb{H}) \\
p=\left(z_{1}, z_{2}\right)
\end{gathered} \begin{array}{ccc}
\widetilde{C}_{2,0}^{s t}(\mathbb{H}) \times[0,+\infty] \times \widetilde{C}_{1,0}^{s t}(\mathbb{H}) \times[0,+\infty] \times \widetilde{C}_{1,0}^{s t}(\mathbb{H}) \times[0,+\infty] & \times\left(\mathfrak{p - x _ { c } ( p )}\right) \\
\left(\frac{i}{\|p\|}, y_{1}\right) & \widetilde{C}_{2}^{s t}(\mathbb{C}) \times[0,+\infty] \\
\left(\mathfrak{i}, y_{2}\right) & \left(\frac{p-z_{c}(p)}{\left|p-z_{c}(p)\right|},\|p\|_{0}=\left|p-z_{c}(p)\right|\right)
\end{array}
$$

so that each codimension 1 boundary stratum can be described explicitly as follows:

(i) The boundary stratum is given by the limit configurations, $p$, with $\|p\|=0$; a generic point in this boundary stratum can be obtained as the $\lambda \rightarrow 0$ limit of a configuration $\left(x_{0}+\lambda z_{1}, x_{0}+\lambda z_{2}\right), x_{0} \in \mathbb{R}, z_{1}, z_{2} \in \mathbb{H}$.

(ii) The boundary stratum is given by the limit configurations, $p$, with $\|p\|_{0}=0,\|p\|, y_{1}, y_{2}$ finite and

non-zero; their image under the projection $\widehat{\mathfrak{C}}_{2,0}(\mathbb{H}) \rightarrow \widetilde{C}_{2,0}^{s t}(\mathbb{H})$ consists of a single point in $\mathbb{H}$; a generic point in this boundary stratum can be obtained as the $\lambda \rightarrow 0$ limit of a configuration $\left(z_{0}+\lambda z_{1}, z_{0}+\lambda z_{2}\right)$ with $z_{0}, z_{1}, z_{2} \in \mathbb{H}$.

(iii) The boundary stratum

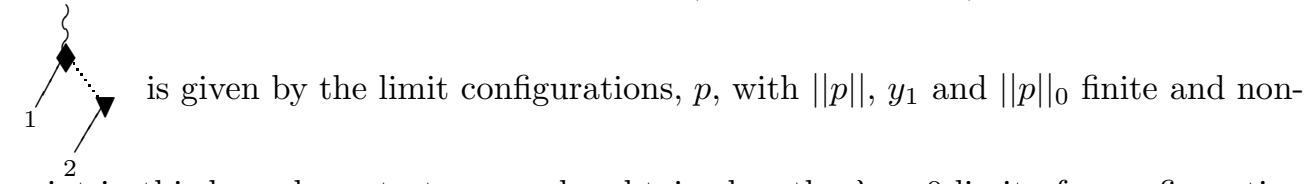
zero while $y_{2}=0$; a generic point in this boundary stratum can be obtained as the $\lambda \rightarrow 0$ limit of a configuration $\left(z_{1}, x_{2}+\mathfrak{i} \lambda y_{2}\right)$ with $z_{1}, z_{2}=x_{2}+\mathfrak{i} y_{2} \in \mathbb{H}$.

(iv) The boundary stratum

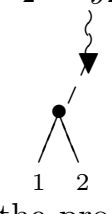
is given by the limit configurations, $p$, with $\|p\|=+\infty$ and $\|p\|_{0}$ a finite

number; their image under the projection $\widehat{\mathfrak{C}}_{2,0} \rightarrow \widetilde{C}_{2,0}^{s t}(\mathbb{H})$ consists of a single point in $\mathbb{H}$; a generic point in this boundary stratum can be obtained as the $\lambda \rightarrow+\infty$ limit of a configuration $\left(\lambda z_{0}+z_{1}, \lambda z_{0}+z_{2}\right), z_{0}, z_{1}, z_{2} \in \mathbb{H}$. 
(v) The boundary stratum

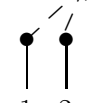

image under the projection $\widehat{\mathfrak{C}}_{2,0}^{1}{ }_{2} \rightarrow \widetilde{C}_{2,0}^{s t}(\mathbb{H})$ consists of two different points in $\mathbb{H}$; a generic point in this boundary stratum can be obtained as the $\lambda \rightarrow+\infty$ limit of a configuration $\left(\lambda z_{1}, \lambda z_{2}\right), z_{1}, z_{2} \in \mathbb{H}$.

(vi) The boundary stratum

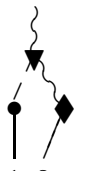

is given by the limit configurations, $p$, with $y_{1}=+\infty, y_{2}$ finite; their image

under the projection $\widehat{\mathfrak{C}}_{2,0} \rightarrow \underset{\widetilde{C}_{2,0}^{s t}}{12}(\mathbb{H})$ consists of two different points in $\overline{\mathbb{H}}$ the first of which lies in $\mathbb{H}$ and the second in $\mathbb{R}$; a generic point in this boundary stratum can be obtained as the $\lambda \rightarrow+\infty$ limit of a configuration $\left(\lambda z_{1}, z_{2}\right)$, $z_{1}, z_{2} \in \mathbb{H}$.

(vii) The boundary stratum

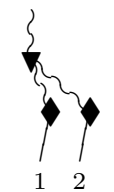

is given by the limit configurations, $p$, with $\|p\|=+\infty, y_{1}$ and $y_{2}$ finite

numbers; their image under the projection $\widehat{\mathfrak{C}}_{2,0} \rightarrow \widetilde{C}_{2,0}^{s t}(\mathbb{H})$ consists of two different points on the real line; a generic point in this boundary stratum can be obtained as the $\lambda \rightarrow+\infty$ limit of a configuration $\left(-\lambda x+\mathfrak{i} y_{1}, \lambda x+y_{2}\right)$, $x \in \mathbb{R}, y_{1}, y_{2} \in \mathbb{R}^{+}$.

A straightforward but tedious inspection of higher codimension strata in $\widehat{\mathfrak{C}}_{2,0}(\mathbb{H})$ tells us that the above classified codimension 1 strata (i)-(vii) are glued together into the following 3-dimensional compact manifold with corners,

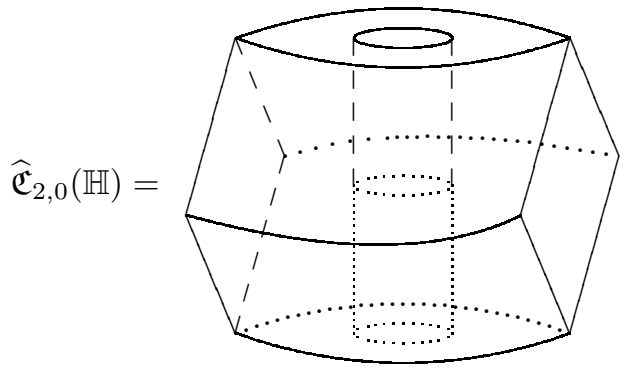

6.3. Semialgebraic structure on $\widehat{\mathfrak{C}}_{n, m}$. The right hand side of the embedding (28) is a product of compact semialgebraic sets. Therefore $\widehat{\mathfrak{C}}_{n, m}$ comes naturally equipped with the structure of a compact semialgebraic set so that we can employ, if necessary, the de Rham algebra of PA differential forms on $\widehat{\mathfrak{C}}_{n, m}$. In fact, $\widehat{\mathfrak{C}}_{n, m}$ is a compact semialgebraic manifold: its smooth semialgebraic atlas can be given by metric trees in a full analogy to $4 \mathbf{4 . 2 . 3}$ and 5.2.1.

6.4. Higher dimensional version. The operad of compactified configuration space $\widehat{\mathfrak{C}}(\mathbb{H})$ has an obvious higher dimensional version, $\widehat{\mathfrak{C}}\left(\mathbb{H}^{d}\right), d \geq 3$, which describes the 4-coloured operad of morphisms of open-closed homotopy Lie algebras.

\section{Operads of Feynman graphs and their representations}

7.1. An operad of Feynman graphs $\mathfrak{G}$. For a finite set $I$ we denote by $G_{I}$ a set of graph $\{14,\{$, with \#I vertices such that

- the edges of $\Gamma$ are directed, beginning and ending at different vertices;

- the vertices of $\Gamma$ are labelled by elements of $I$, i.e. a bijection $V(\Gamma) \rightarrow I$ is fixed;

- the set of edges, $E(\Gamma)$, is totally ordered.

\footnotetext{
14 A graph $\Gamma$ is, by definition, a 1-dimensional $C W$-complex whose 0-cells are called vertices and 1-dimensional cells are called edges. Its automorphism group as a $C W$-complex is denoted by $A u t(\Gamma)$.
} 
We identify two total orderings on the set $E(\Gamma)$ (that is, isomorphisms $E(\Gamma) \simeq[\# E(\Gamma)]$ ), if they differ by an even permutation of $[\# E(\Gamma)]$. Thus there are precisely two possible ordering 15 on the set $E(\Gamma)$ and the group $\mathbb{Z}_{2}$ acts freely on $G_{I}$ by ordering changes; its orbit is denoted by $\left\{\Gamma, \Gamma_{\text {opp }}\right\}$. If $I=[n]$, we write $G_{n}$ for $G_{I}$. The subset of $G_{n}$ consisting of graphs with precisely $l$ edges is denoted by $G_{n, l}$.

For any fixed integer $d \in \mathbb{Z}$ we first set $\mathbb{K}\left\langle G_{n, l}\right\rangle$ to be the vector space spanned by isomorphism classes, $[\Gamma]$ of graphs $\Gamma \in G_{n, l}$ modulo the relation $\left[\Gamma_{o p p}\right]=(-1)^{d-1}[\Gamma]$, and then define a $\mathbb{Z}$-graded $\mathbb{S}_{n}$-module,

$$
\mathfrak{G}(n):=\bigoplus_{l=0}^{\infty} \mathbb{K}\left\langle G_{n, l}\right\rangle[(1-d) l] .
$$

Note that if $d$ is even, then any graph $\Gamma \in G_{n, l}$ which has a pair of vertices connected by two or more edges with the same orientation vanishes in $\mathfrak{G}(n)$; for example, $\bullet=0$ in $\mathfrak{G}(2)$ for $d$ even. If $\Gamma$ does not vanish, then its degree in $\mathfrak{G}(n)$ is equal to $(d-1) \# E(\Gamma)$, i.e. every edge contributes $d-1$ to the total degree.

The resulting $\mathbb{S}$-module,

$$
\mathfrak{G}=\{\mathfrak{G}(n)\}_{n \geq 1}
$$

has a natural operad structure defined as follows: let $[n] \rightarrow[p]$ be an arbitrary surjection, and let $[n]=I_{1} \sqcup I_{2} \sqcup \ldots \sqcup I_{p}$ be the associated partition of $[n]$, then the map

$$
\begin{array}{ccc}
\circ: \quad \mathfrak{G}(p) \otimes \mathfrak{G}\left(I_{1}\right) \otimes \ldots \otimes \mathfrak{G}\left(I_{p}\right) & \longrightarrow & \mathfrak{G}(n) \\
\left(\Gamma_{0}, \Gamma_{1}, \ldots, \Gamma_{p}\right) & \longrightarrow & \Gamma_{0} \circ\left(\Gamma_{1} \otimes \ldots \otimes \Gamma_{p}\right)
\end{array}
$$

is given, by definition, by

$$
\Gamma_{0} \circ\left(\Gamma_{1} \otimes \ldots \otimes \Gamma_{p}\right)=\sum_{\Gamma \in G_{I_{1}, \ldots, I_{p}}}(-1)^{\sigma_{\Gamma}} \Gamma
$$

where the summation runs over a subset $G_{I_{1}, \ldots, I_{p}} \subset G_{n}$ consisting of all graphs $\Gamma$ satisfying the following condition: the subgraph $16 \Gamma_{I_{1}}, \Gamma_{I_{2}}, \ldots, \Gamma_{I_{p}}$, of $\Gamma$ spanned by the vertices labelled, respectively, by the subsets $I_{1}, I_{2}, \ldots, I_{p} \subset$ $[n]$ are isomorphic, respectively, to $\Gamma_{1}, \Gamma_{2}, \ldots, \Gamma_{p}$, and the quotient graph, $\Gamma /\left\{\Gamma_{I_{1}}, \Gamma_{I_{2}}, \ldots, \Gamma_{I_{p}}\right\}$ is isomorphic to $\Gamma_{0}$. The sign is determined by the equality $e_{\Gamma /\left\{\Gamma_{I_{1}}, \Gamma_{I_{2}}, \ldots, \Gamma_{I_{p}}\right\}} e_{\Gamma_{I_{1}}} e_{\Gamma_{I_{2}}} \cdots e_{\Gamma_{I_{p}}}=(-1)^{\sigma_{\Gamma}} e_{\Gamma}$ (see footnote 15), i.e. by comparing the orderings on the sets of edges. The unique element in $G_{1,0}$ serves as a unit in this operad. For example,

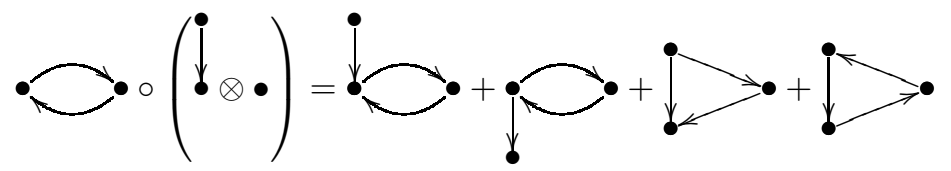

where orientations of graphs on the r.h.s. are chosen implicitly in a such a way that they contribute with coefficient +1 . Thus the operadic composition, $\Gamma_{0} \circ\left(\Gamma_{1} \otimes \ldots \otimes \Gamma_{p}\right)$ is given simply by substitutions of the graphs $\Gamma_{1}, \ldots, \Gamma_{p}$ into the vertices of the graph $\Gamma_{0}$ and redistributing the edges in all possible ways.

The number $d-1$ is called the propagator degree of the operad $\mathfrak{G}$. Perhaps we should have imprinted $d$ somehow into the notation, say use the symbol $\mathfrak{G}[d]$ to indicate its dependence on $d$, but we do not bother doing it as in applications the propagator degree will be always clear from the context.

7.1.1. Coloured variants of $\mathfrak{G}$. Let a $\mathbb{Z}$-graded $\mathbb{S}_{n}$-module $\bigoplus_{\substack{n=n_{1}+n_{2} \\ 2 n_{1}+n_{2} \geq 2}} \mathrm{G}^{\uparrow \downarrow}\left(n_{1}, n_{2}\right)$ be defined as $\mathfrak{G}(n)$ above except that the vertices of graphs $\Gamma$ from $\mathrm{G}^{\uparrow \downarrow}\left(n_{1}, n_{2}\right)$ are coloured in one of two possible colours, say white and black, i.e. $V(\Gamma)$ comes equipped with a splitting into a disjoint union $V(\Gamma)=V_{w}(\Gamma) \sqcup V_{b}(\Gamma), \# V_{w}(\Gamma)=n_{1}$ and $\# V_{b}(\Gamma)=n_{2}$, satisfying the condition $2 n_{1}+n_{2} \geq 2$. It is useful to visualize such a graph as drawn on the closed

\footnotetext{
${ }^{15}$ It is useful sometimes to identify an orientation of $\Gamma \in \mathcal{G}_{I}$ with a vector $e_{\Gamma}:=\wedge_{e \in E(\Gamma)} e$ in the real one dimensional vector space $\wedge^{l} \mathbb{R}[E(\Gamma)]$, where $\mathbb{R}[E(\Gamma)]$ is the $l$-dimensional vector space spanned over $\mathbb{R}$ by the set $E(\Gamma)$.

${ }^{16}$ For any subset $A \subset[n]$ and any graph $\Gamma$ in $G_{n}$ there is an associated subgraph $\Gamma_{A}$ of $\Gamma$ whose vertices are, by definition, those vertices of $\Gamma$ which are labelled by elements of $A$, and whose edges are all the edges of $\Gamma$ which connect these $A$-labelled vertices. If we compress all the $A$-labelled vertices of $\Gamma$ (together with all the edges connecting these $A$-labelled vertices) into a single vertex, then we obtain from $\Gamma$ a new graph which we denote by $\Gamma / \Gamma_{A}$. Analogously one defines the quotient graph $\Gamma /\left\{\Gamma_{I_{1}}, \ldots, \Gamma_{I_{p}}\right\}$ for any partition $V(\Gamma)=I_{1} \sqcup \ldots \sqcup I_{p}$
} 
upper half plane $\overline{\mathbb{H}}$ with white vertices placed in $\mathbb{H}$ and black vertices on the boundary $\mathbb{R}$ in the order which is consistent with their numbering, e.g.

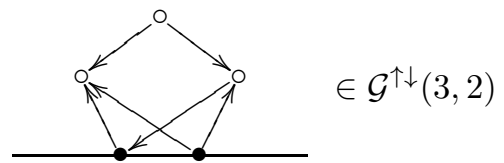

The S-bimodule

$$
\mathfrak{G}^{\uparrow \downarrow}=\left\{\mathfrak{G}(n) \oplus \bigoplus_{n=n_{1}+n_{2}} \mathcal{G}^{\uparrow \downarrow}\left(n_{1}, n_{2}\right)\right\}_{n \geq 2,2 n_{1}+n_{2} \geq 2}
$$

is naturally a 2-coloured operad with respect to substitutions of

(i) graphs from $\mathfrak{G}(n)$ into the vertices of $\mathfrak{G}(m)$ and into the white vertices of $\mathcal{G}^{\uparrow \downarrow}\left(m_{1}, m_{2}\right)$,

(ii) graphs from $\mathcal{G}^{\uparrow \downarrow}\left(n_{1}, n_{2}\right)$ into the black vertices of $\mathcal{G}^{\uparrow \downarrow}\left(m_{1}, m_{2}\right)$.

One can consider versions, $\mathcal{G}^{\downarrow}$ and $\mathcal{G}^{\uparrow}$, of the 2-coloured operad $\mathcal{G}^{\uparrow \downarrow}$ spanned by graphs $\Gamma$ such that all edges incident to any black vertex are oriented towards (respectively, outwards) that black vertex. For example

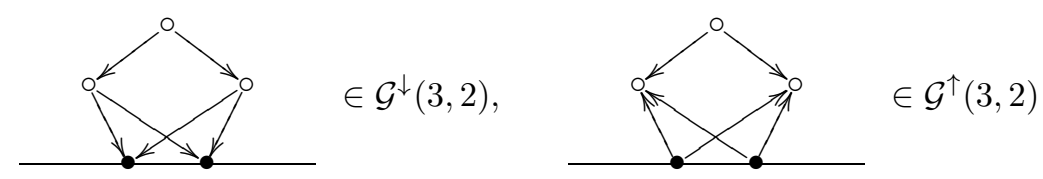

More generally, with any compactified configuration space, $\bar{C}=\left\{\bar{C}_{n}\right\}$, considered in $\S 2-4$ of this paper one can associate a coloured operad of Feynman graphs, $\mathfrak{G}_{\bar{C}}=\left\{\mathfrak{G}_{\bar{C}}(n)\right\}$, whose elements are, by definition, linear combinations of graphs from $\mathfrak{G}(n)$ (or from an appropriate subset of $\mathfrak{G}(n)$ ) whose vertices are identified with configuration of points in $\bar{C}_{n}$ and hence are coloured correspondingly. For example, the above operad $\mathfrak{G}$ with propagator degree $d-1$ can be re-denoted as $\mathfrak{G}_{\bar{C}\left(\mathbb{R}^{d}\right)}$ while any of the operads $\mathfrak{G}^{\uparrow \downarrow}, \mathfrak{G}^{\downarrow}$ and $\mathfrak{G}^{\uparrow}$ can be associated with the configuration spaces $\bar{C}\left(\mathbb{H}^{d}\right)$ and hence can be re-denoted by $\mathfrak{G}_{\bar{C}\left(\mathbb{H}^{d}\right)}^{\uparrow \downarrow}, \mathfrak{G}_{\bar{C}\left(\mathbb{H}^{d}\right)}^{\downarrow}$ and $\mathfrak{G}_{\bar{C}\left(\mathbb{H}^{d}\right)}^{\uparrow}$ respectively. It is the operad $\mathfrak{G}_{\bar{C}}^{\downarrow}\left(\mathbb{H}^{d}\right)$ which Kontsevich used in his paper [Ko2] on the formality theorem; we shall consider below other two operads as well.

7.1.2. Dual cooperads of Feynman graphs. As the vector space $\mathbb{K}\left\langle G_{n, l}\right\rangle$ is finite-dimensional for each $n$ and $l$, the dual $\mathbb{S}$-modules,

$$
\mathfrak{G}^{*}:=\left\{\operatorname{Hom}\left(\mathfrak{G}_{k}(n), \mathbb{K}\right)\right\}_{n \geq 1}, \quad\left(\mathfrak{G}^{\uparrow \downarrow}\right)^{*}:=\left\{\operatorname{Hom}\left(\mathfrak{G}^{\uparrow \downarrow}(n), \mathbb{K}\right)\right\}_{n \geq 1}, \quad \text { etc. }
$$

have induced structures of (coloured) cooperads in the category of graded vector spaces. these spaces come equipped canonically with distinguished bases which we can identify with graphs. We denote by $\check{\mathfrak{G}}$, $\mathfrak{G}^{\uparrow \uparrow}$, etc. their subcooperads spanned by finite linear combinations of such graphs.

For example, the dualization of the operadic composition (33) in $\mathfrak{G}$ gives the following formula for the co-composition in the cooperad $\check{\mathfrak{G}}$,

$$
\begin{aligned}
& \Delta: \quad \check{\mathfrak{G}}(n) \quad \longrightarrow \quad \bigoplus_{\substack{\left.[n]=I_{1}\right\lrcorner \ldots \sqcup \cup I_{p} \\
1 \leq p \leq n}} \check{\mathfrak{G}}(p) \otimes \check{\mathfrak{G}}\left(I_{1}\right) \otimes \check{\mathfrak{G}}\left(I_{2}\right) \otimes \ldots \otimes \check{\mathfrak{G}}\left(I_{p}\right) \\
& \Gamma \quad \longrightarrow \quad \Delta(\Gamma):=\sum_{p=0}^{n-1} \sum_{[n]=I_{1} \sqcup \ldots \sqcup I_{p}}(-1)^{\varepsilon} \Gamma /\left\{\Gamma_{I_{1}}, \ldots, \Gamma_{I_{p}}\right\} \otimes \Gamma_{I_{1}} \otimes \Gamma_{I_{2}} \otimes \ldots \otimes \Gamma_{I_{p}} .
\end{aligned}
$$

where the summation runs over all possible partitions of $[n]$. As $\mathfrak{G}$ is unital, we can equivalently describe cooperad structure in $\check{\mathfrak{G}}_{d}$ in terms of the reduced co-composition, $\forall i \in[n]$,

$$
\begin{aligned}
\Delta_{i}^{r e d}: \quad \check{\mathfrak{G}}(n) & \longrightarrow \bigoplus_{i \in A \subset[n]} \check{\mathfrak{G}}(n-\# A+1) \otimes \check{\mathfrak{G}}(A) \\
\Gamma & \longrightarrow \Delta_{i}^{r e d}(\Gamma):=\sum_{i \in A \subset[n]}(-1)^{\varepsilon} \Gamma / \Gamma_{A} \otimes \Gamma_{A} .
\end{aligned}
$$


Setting $\check{\mathfrak{G}}(1)=0$, we make the data $\left(\left\{\check{\mathfrak{G}}_{d}(n)\right\}_{n \geq 2}\right.$ into a non-unital pseudo-cooperad which we denote by the same symbol $\breve{G}$ but call the non-unital pseudo-cooperad of Feynman graphs. Analogously one defines non-unital pseudo-cooperads $\check{\mathfrak{G}}^{\uparrow \downarrow}$ etc.

7.2. A class of representations of $\mathfrak{G}$. Note that operads of Feynman graphs depend on the choice of a parameter $d$ which we do not show in the notations. Any representation of $\mathfrak{G}$ in a graded vector space $X$,

$$
\rho: \mathfrak{G} \longrightarrow \mathcal{E} n d_{X}
$$

is uniquely determined by its values on the generators, $\Gamma \in G_{n, l}$, i.e. by a collection of operators,

$$
\left\{\Phi_{\Gamma}:=\rho(\Gamma) \in \operatorname{Hom}_{(d-1) \# E(\Gamma)}\left(X^{\otimes \# V(\Gamma)}, X\right)\right\}
$$

which, in accordance with (34), satisfy the equations

$$
\begin{gathered}
(-1)^{K} \Phi_{\Gamma_{0}}\left(\Phi_{\Gamma_{1}}\left(x_{1}, \ldots, x_{n_{1}}\right), \Phi_{\Gamma_{2}}\left(x_{n_{1}+1}, \ldots, x_{n_{1}+n_{2}}\right), \ldots, \Phi_{\Gamma_{p}}\left(x_{n_{1}+\ldots+n_{p-1}+1}, \ldots, x_{n_{1}+\ldots+n_{p}}\right)\right)= \\
\sum_{\Gamma \in \mathfrak{G}_{d}\left(n_{1}, \ldots, n_{p}\right)}(-1)^{\sigma(\Gamma)} \Phi_{\Gamma}\left(x_{1}, x_{2}, \ldots, x_{n_{1}+\ldots+n_{p}}\right)
\end{gathered}
$$

for any $\Gamma_{i} \in G_{n_{i}, l_{i}}, i=0,1, \ldots, p$, and any $x_{1}, x_{2}, \ldots, x_{n_{1}+\ldots+n_{p}} \in A$. Here $(-1)^{K}$ stands for the usual Koszul sign arising under a composition of homogeneous maps and $\mathfrak{G}\left(n_{1}, \ldots, n_{p}\right) \subset \mathfrak{G}\left(n_{1}+\ldots+n_{p}\right)$ is a subset consisting of all graphs $\Gamma$ whose subgraphs, $\Gamma_{I_{1}}, \Gamma_{I_{2}}, \ldots, \Gamma_{I_{p}}$, spanned by vertices labelled, respectively, by

$$
I_{1}:=\left\{1, \ldots, n_{1}\right\}, I_{2}:=\left\{n_{1}+1, \ldots, n_{1}+n_{2}\right\}, \ldots, I_{p}:=\left\{n_{1}+\ldots+n_{p-1}+1, \ldots, n_{1}+\ldots+n_{p}\right\}
$$

are isomorphic to $\Gamma_{1}, \Gamma_{2}, \ldots, \Gamma_{p}$, and the quotient graph, $\Gamma /\left\{\Gamma_{1}, \Gamma_{2}, \ldots, \Gamma_{p}\right\}$, is isomorphic to $\Gamma_{0}$.

Let $W$ be a $\mathbb{Z}$-graded vector space and $\tau \in W^{*} \otimes W^{*}$ a non zero element of degree $d-1$. Any element $\omega \in W^{*}$ defines a map $W \rightarrow \mathbb{K}$ which can be uniquely extended to a derivation of the symmetric tensor algebra $\bigodot^{\bullet} W$. Therefore, the element $\tau$ gives naturally rise to a biderivation on $\bigodot^{\bullet} W \otimes \bigodot^{\bullet} W$ which we denote by $\Delta^{\tau}$. Moreover, for any $n \geq 2$ and any ordered pair of different integers $i, j \in[n], \tau$ gives rise to an automorphism,

$$
\Delta_{i j}^{\tau}:(\odot \cdot W)^{\otimes n} \longrightarrow(\odot \cdot W)^{\otimes n}
$$

which acts as $\Delta^{\tau}$ on $i$-th and $j$-th tensor factors and as the identity on all other tensor factors. Next, for any graph $\Gamma \in G_{n, l}$, we define

$$
\Phi_{\Gamma}:\left(\odot{ }^{\bullet} W\right)^{\otimes n} \longrightarrow \odot \odot^{\bullet} W
$$

as the composition,

$$
\Phi_{\Gamma}:=\mu^{(n)} \circ \prod_{e \in E(V)} \Delta_{I n(e), \text { Out }(e)}^{\tau},
$$

where, for an edge $e$ connecting a vertex labelled by $i \in[n]$ to a vertex labelled by $j$, we set $\Delta_{I n(e), \text { Out }(e)}^{\tau}:=\Delta_{i, j}^{\tau}$, and $\mu^{(n)}$ stands for the natural multiplication,

$$
\mu^{(n)}: \begin{array}{ccc}
(\odot \cdot W)^{\otimes n} & \longrightarrow & \odot \bullet W \\
\left(f_{1}, f_{2}, \ldots, f_{n}\right) & \longrightarrow & f_{1} f_{2} \cdots f_{n} .
\end{array}
$$

7.2.1. Proposition. For any graded vector space $W$ and any element $\tau \in W^{*} \otimes W^{*}$ of degree $k$ the operators (38) define a representation of the operad of Feynman graphs in the vector space $X:=\odot{ }^{\bullet} W$.

Proof. One has only to check equations (37) but this is straightforward due to the Leibniz rule. 
7.2.2. Poisson-Schouten algebras. Let $V$ be an arbitrary $\mathbb{Z}$-graded vector space $V$ and let

$$
W_{d}:=V^{*}[2-d] \oplus V[-1] .
$$

Then $W_{d}^{*} \otimes W_{d}^{*}$ contains a distinguished element $\tau:=s^{1-d} \operatorname{Id}_{V}$ such that

$$
\Delta^{\tau}=\sum_{\alpha} \frac{\partial}{\partial \psi_{\alpha}} \otimes \frac{\partial}{\partial x^{\alpha}}
$$

where, for a basis $\left\{e_{\alpha}\right\}$ in $V$ and the associated dual basis, $\left\{e^{\alpha}\right\}$, in $V^{*}$, we set

$$
x^{\alpha}:=s^{2-d} e^{\alpha}, \quad \psi_{\alpha}:=s^{-1} e_{\alpha} .
$$

This operator makes the completed graded commutative algebra $\widehat{\odot} W_{d} \simeq \mathbb{K}\left[\left[x^{\alpha}, \psi_{\alpha}\right]\right]$ into a $d$-algebra (in the terminology of [Ko3] ) with the degree $1-d$ Lie brackets given by,

$$
\{f \bullet g\}_{1-d}=\sum_{\alpha} \frac{f \overleftarrow{\partial}}{\partial \psi_{\alpha}} \frac{\vec{\partial} g}{\partial x^{\alpha}}+(-1)^{|f||g|+(d-1)(f+g)+d} \frac{g \overleftarrow{\partial}}{\partial \psi_{\alpha}} \frac{\vec{\partial} f}{\partial x^{\alpha}}
$$

We call this particular class of $d$-algebras,

$$
\mathfrak{g}_{d}(V):=\left(\widehat{\odot} W_{d},\{,\}_{1-d}\right),
$$

the Poisson-Schouten algebras. Note that

$$
\mathfrak{g}_{2}(V)=\mathcal{T}_{\text {poly }}(V)
$$

can be identified with the Schouten algebra of polyvector fields on $V$ viewed as an affine or formal manifold.

By Proposition 7.2.1 every such an algebra comes equipped with a representation of the operad $\mathfrak{G}$ (which, as we shall see below, can be quantized). Let us have a brief look at the set of Maurer-Cartan elements of $\mathfrak{g}_{d}(V)$,

$$
\mathcal{M C}(V):=\left\{\gamma \in \mathfrak{g}_{d}(V) \mid\{\gamma \bullet \gamma\}_{1-d}=0 \text { and }|\gamma|=d\right\},
$$

for the case $V=\mathbb{R}^{d}$ and $d \geq 2$ :

$d=2$ : the set $\mathcal{M C}_{2}(V)$ consists of formal Poisson structures on $V$, that is, $\mathcal{M C}_{2}(V)$ is the set of formal bi-vector fields $\gamma \in \wedge^{2} \mathcal{T}_{V}$ satisfying the equation $\{\gamma \bullet \gamma\}_{-1}=0$; the 2-algebra $\mathfrak{g}_{2}\left(\mathbb{R}^{d}\right)$ is precisely the Schouten algebra of formal polyvector fields on $\mathbb{R}^{d}$;

$d=3$ : a generic element of degree 3 has the form,

$$
\gamma=\sum_{\alpha, \beta, \gamma} \underbrace{C_{\alpha \beta \gamma}}_{\wedge^{3} V \rightarrow K} x^{\alpha} x^{\beta} x^{\gamma}+\sum_{\alpha, \beta, \gamma} \underbrace{C_{\alpha \beta}^{\gamma}}_{\wedge^{2} V \rightarrow V} x^{\alpha} x^{\beta} \psi_{\gamma}+\sum_{\alpha, \beta, \gamma} \underbrace{C_{\gamma}^{\alpha \beta}}_{V \rightarrow \wedge^{2} V} \psi_{\alpha} \psi_{\beta} x^{\gamma}+\sum_{\alpha, \beta, \gamma} \underbrace{C^{\alpha \beta \gamma}}_{\mathbb{K} \rightarrow \wedge^{3} V} \psi_{\alpha} \psi_{\beta} \psi_{\gamma} .
$$

It is easy to check that such an element satisfies the equation $\{\gamma \bullet \gamma\}_{-2}=0$ if and only if the associated set of degree 0 maps,

$$
\mathbb{K} \rightarrow \wedge^{3} V, \quad V \rightarrow \wedge^{2} V, \quad \wedge^{2} V \rightarrow V, \quad \wedge^{3} V \rightarrow \mathbb{K},
$$

make $V$ into a Lie quasi-bialgebra. This notion was introduced by Drinfeld in order ro describe infinitesimal breaking of the (co)associativity of basic bialgebra operations due to associators.

$d=4$ : the set $\mathcal{M C}_{4}(V)$ describes triples, $([], g,, \Phi)$, consisting of a Lie algebra structure on $V^{*}$, and Lie invariant elements $g \in \odot{ }^{2} V^{*}$ and $\Phi \in \wedge^{4} V$,

$$
\gamma=\sum_{\alpha, \beta} \underbrace{C_{\alpha \beta}}_{g: \odot^{2} V \rightarrow \mathbb{K}} x^{\alpha} x^{\beta}+\sum_{\alpha, \beta, \gamma} \underbrace{C_{\gamma}^{\alpha \beta}}_{V \rightarrow \wedge^{2} V} \psi_{\alpha} \psi_{\beta} x^{\gamma}+\sum_{\alpha, \beta, \gamma, \varepsilon} \underbrace{C^{\alpha \beta \gamma \varepsilon}}_{\mathbb{K} \rightarrow \wedge^{4} V} \psi_{\alpha} \psi_{\beta} \psi_{\gamma} \psi_{\varepsilon} .
$$

$d \geq 5$ : the set $\mathcal{M C}_{d}(V)$ describes pairs, $([],, \Phi)$, consisting of a Lie algebra structure on $V^{*}$ and a Lie invariant element $\Phi \in \wedge^{d} V$. 
7.3. A class of representations of the 2-coloured operads $\mathfrak{G}^{\uparrow \downarrow}, \mathfrak{G}^{\uparrow}$ and $\mathfrak{G}^{\downarrow}$. Formulae (38) for $\tau:=s^{1-d} \operatorname{Id}_{V}$ give a representation of the 2 -coloured operad

$$
\rho: \mathfrak{G}^{\uparrow \downarrow} \longrightarrow \mathcal{E} n d_{\left\{X_{1}, X_{2}\right\}}
$$

in $X_{1}=X_{2}=\mathfrak{g}_{d}(V)$. We shall quantize this representation in $\$ 9.3$ using the standard homogeneous volume form on the sphere $S^{d-1}$ as a propagator.

The Poisson-Schouten algebra $\mathfrak{g}_{d}(V)$ contains two graded commutative subalgebras, $\odot \bullet\left(V^{*}[2-d]\right)$ and $\odot \bullet(V[-1])$, which are also Abelian Lie subalgebras. There are natural projections,

$$
\pi_{\downarrow}: \mathfrak{g}_{d}(V) \rightarrow \odot \bullet\left(V^{*}[2-d]\right) \text {, and } \pi_{\uparrow}: \mathfrak{g}_{d}(V) \rightarrow \odot \bullet(V[-1]) .
$$

If $\Gamma \in \mathcal{G}^{\downarrow}$ has $n$ white vertices and $m$ black vertices, then we set,

$$
\begin{array}{ccc}
\Phi_{\Gamma}: \quad\left(\odot \bullet\left(V^{*}[2-d] \oplus V[-1]\right)^{\otimes n}\right) \otimes\left(\odot \bullet\left(V^{*}[2-d]\right)^{\otimes m}\right. & \longrightarrow & \odot \bullet\left(V^{*}[2-d]\right) \\
\gamma_{1} \otimes \ldots \otimes \gamma_{n} \otimes f_{1} \otimes \ldots \otimes f_{m} & \longrightarrow & \Phi_{\Gamma}\left(\gamma_{1}, \ldots, \gamma_{n}, f_{1}, \ldots, f_{m}\right)
\end{array}
$$

where

$$
\Phi_{\Gamma}\left(\gamma_{1}, \ldots, \gamma_{n}, f_{1}, \ldots, f_{m}\right):=\pi_{\downarrow} \circ \mu^{(n+m)} \circ \prod_{e \in E(V)} \Delta_{I n(e), \text { Out }(e)}^{\tau}\left(\gamma_{1} \otimes \cdots \otimes \gamma_{n} \otimes f_{1} \otimes \cdots \otimes f_{m}\right) .
$$

It is easy to check that for any graded vector space $V$ the operators (38) and (40) define a representation of the 2coloured operad of Feynman graphs $\mathfrak{G}^{\downarrow}$ in the vector spaces $X_{c}:=\odot^{\bullet}\left(V^{*}[2-d] \oplus V[-1]\right)$ and $X_{o}=\odot \bullet\left(V^{*}[2-d]\right)$. Quantization of this representation in the case $d=2$ with the help of the Kontsevich propagator gives us his formality map (see below).

Replacing in the above construction $\odot{ }^{\bullet}\left(V^{*}[2-d]\right)$ by $\odot \bullet(V[-1])$ and $\pi_{\downarrow}$ by $\pi_{\uparrow}$ one obtains a canonical representation of $\mathfrak{G}^{\uparrow}$ in the vector spaces $X_{c}:=\odot{ }^{\bullet}\left(V^{*}[2-d] \oplus V[-1]\right)$ and $X_{o}=\odot \bullet(V[-1])$.

\section{De Rham field theories on configuration spaces and quantization}

8.1. Completed tensor product of de Rham algebras. Let Man be the category of smooth manifolds with corners (or semialgebraic manifolds) and let $\Omega_{\mathcal{M} a n}$ be the associated category 17 of de Rham algebras of (PA) differential forms. The category Man is symmetric monoidal with respect to the ordinary Cartesian product, $\times$, of manifolds. We shall define a completed tensor product of de Rham algebras as follows,

$$
\Omega_{X_{1}} \widehat{\otimes} \Omega_{X_{2}}:=\Omega_{X_{1} \times X_{2}},
$$

where $X_{1}$ and $X_{2}$ are arbitrary objects in $\mathcal{M} a n$. This completed tensor product makes $\Omega_{\mathcal{M} a n}$ into a symmetric monoidal category so that we can define operads and cooperads in $\Omega_{\mathcal{M} a n}$. Moreover, in this case one can associate with an arbitrary operad $\mathcal{P}=\{\mathcal{P}(n)\}$ in the category $\mathcal{M}$ an a co-operad, $\Omega_{\mathcal{P}}:=\left\{\Omega_{\mathcal{P}(n)}\right\}$, of de Rham algebras in the category $\Omega_{\mathcal{M} a n}$.

8.2. De Rham field theory on $\bar{C}\left(\mathbb{R}^{d}\right)$. For any proper subset $A \subset[n]$ of cardinality at least two there is a uniquely associated operadic composition 18 ,

$$
\circ_{A}:=\circ_{\bullet}^{([n]-A) \sqcup \bullet, A}: \bar{C}_{([n]-A) \sqcup \bullet}\left(\mathbb{R}^{d}\right) \times \bar{C}_{A}\left(\mathbb{R}^{d}\right) \longrightarrow \bar{C}_{n}\left(\mathbb{R}^{k+1}\right),
$$

which sends the Cartesian product into a corresponding boundary component in $\partial \bar{C}_{n}\left(\mathbb{R}^{d}\right)$. Moreover,

$$
\partial \bar{C}_{n}\left(\mathbb{R}^{d}\right)=\bigcup_{A \mp[n], \# A \geq 2} \circ_{A}\left(\bar{C}_{([n]-A) \sqcup \bullet}\left(\mathbb{R}^{d}\right) \times \bar{C}_{A}\left(\mathbb{R}^{d}\right)\right)
$$

Let $\Omega_{\bar{C}_{n}\left(\mathbb{R}^{d}\right)}=\oplus_{p \geq 0} \Omega_{\bar{C}_{n}\left(\mathbb{R}^{d}\right)}^{p}$ stand for the de Rham algebra of $(P A)$ smooth complex valued differential forms on the $\bar{C}_{n}\left(\mathbb{R}^{d}\right)$. The associated dg $\mathbb{S}$-module,

$$
\Omega_{\bar{C}\left(\mathbb{R}^{d}\right)}:=\left\{\Omega_{\bar{C}_{n}\left(\mathbb{R}^{d}\right)}, d_{D R}\right\}_{n \geq 2}
$$

is naturally a non-unital dg cooperad in the category $\Omega_{\mathcal{M} a n}$.

\footnotetext{
${ }^{17}$ One might prefer using the language of functors and natural transformations in this subsection, but, to save the space and the time, we choose to consider $\Omega_{\mathcal{M} \text { an }}$ not as a functor but as a subcategory of the category of dg algebras.

${ }^{18}$ See Section A.3.5 in Appendix for explanation of the notation $\circ_{\bullet}^{([n]-A) \sqcup \bullet, A}$.
} 
8.2.1. Definition. A de Rham field theory on the operad $\left\{\bar{C}_{n}\left(\mathbb{R}^{d}\right)\right\}_{n \geq 1}$ is a morphism 19,

$$
\Omega:(\check{\mathfrak{G}}, 0) \longrightarrow\left(\Omega_{\bar{C}\left(\mathbb{R}^{d}\right)}, d_{D R}\right),
$$

of dg cooperads.

Let us translate this definition into a system of explicit formulae.

8.2.2. Proposition. A de Rham field theory on $\left\{\bar{C}_{n}\left(\mathbb{R}^{d}\right)\right\}_{n \geq 1}$ is equivalent to a family of maps

$$
\left\{\begin{array}{ccc}
\Omega: G_{n, l} & \longrightarrow & \Omega_{\bar{C}_{n}\left(\mathbb{R}^{d}\right)}^{l(d-1)} \\
\Gamma & \longrightarrow & \Omega_{\Gamma}
\end{array}\right\}_{n \geq 1, l \geq 0},
$$

such that $d_{D R} \Omega_{\Gamma}=0, \Omega_{\Gamma_{\text {opp }}}=-(-1)^{d-1} \Omega_{\Gamma}$, and, for any boundary stratum in $\bar{C}_{n}\left(\mathbb{R}^{d}\right)$ given by the image of an operadic composition 41, one has

$$
\circ_{A}^{*}\left(\Omega_{\Gamma}\right)=(-1)^{\varepsilon} \Omega_{\Gamma / \Gamma_{A}} \wedge \Omega_{\Gamma_{A}}
$$

where the sign is determined by the equality $e_{\Gamma / \Gamma_{A}} e_{\Gamma_{A}}=(-1)^{\varepsilon} e_{\Gamma}$.

Proof. As differential in $\check{\mathfrak{G}}$ is trivial, the image of $\Omega$ belongs to the subspace of closed differential forms. As the co-composition in $\mathfrak{G}$ is given by (35) while the co-composition in the cooperad $\Omega_{\bar{C}\left(\mathbb{R}^{d}\right)}$,

$$
\begin{aligned}
\Delta: \Omega_{\bar{C}\left(\mathbb{R}^{d}\right)} & \longrightarrow \\
\omega & \longrightarrow \Delta(\omega):=\bigoplus_{A \subsetneq[n], \# A \geq 2} \Omega_{\bar{C}_{([n]-A) \sqcup}\left(\mathbb{R}^{d}\right)} \hat{\otimes} \Omega_{\bar{C}_{A}\left(\mathbb{R}^{d}\right)} \\
& \left.\bigoplus_{A} \circ_{A}^{*}(\omega)\right|_{\bar{C}_{([n]-A) \sqcup} \cdot\left(\mathbb{R}^{d}\right) \times \bar{C}_{A}\left(\mathbb{R}^{d}\right)}
\end{aligned}
$$

is just a direct sum of restrictions of the differential form $\omega$ to all the boundary components $\operatorname{Im}\left(\circ_{A}\right)$, we conclude that the map $\Omega$ is a morphism of cooperads if and only if equations (42) hold for any $n$ and any $A \varsubsetneqq[n]$ with $\# A \geq 2$.

8.2.3. Theorem. Let

$$
\begin{array}{rlll}
\rho: & \mathfrak{G} & \longrightarrow & \mathcal{E} n d_{X} \\
& \Gamma \longrightarrow & \Phi_{\Gamma}
\end{array}
$$

be a representation of the operad of Feynman graphs in a graded vector space $X$. Then any de Rham field theory on $\bar{C}\left(\mathbb{R}^{d}\right)$ makes $X$ into a representation,

$$
\begin{array}{clc}
\rho_{Q}:\left(\mathcal{F C h a i n s}\left(\bar{C}\left(\mathbb{R}^{d}\right)\right), \partial\right) & \longrightarrow & \mathcal{E} n d_{X} \\
\bar{C}_{n}\left(\mathbb{R}^{d}\right) & \longrightarrow & \mu_{n} \in \operatorname{Hom}\left(X^{\otimes n}, X\right)
\end{array}
$$

of the operad of fundamental chains of $\bar{C}\left(\mathbb{R}^{d}\right)$, that is, makes $X$ into a $\mathcal{L}_{\infty}\{d-1\}$-algebra with operations, $\left\{\mu_{n}\right.$ : $\left.\otimes^{n} X \rightarrow X\right\}_{n \geq 2}$ given by

$$
\mu_{n}:=\left\{\begin{array}{cl}
\sum_{\Gamma \in G_{n, \frac{n d-2}{d-1}-1}} c_{\Gamma} \Phi_{\Gamma} & \text { if } d-1 \mid n d-2 \\
0 & \text { otherwise. }
\end{array}\right.
$$

wher 20

$$
c_{\Gamma}:=\int_{\bar{C}_{n}\left(\mathbb{R}^{d}\right)} \Omega_{\Gamma}
$$

\footnotetext{
${ }^{19}$ Strictly speaking, the objects on both sides of the arrow belong to different symmetric monoidal categories as the tensor product on the r.h.s. is completed; however, it is straightforward to adopt axioms behind the ordinary notion of a morphism of operads in the category of vector spaces to this particular case.

${ }^{20} c_{\Gamma}$ and $\Phi_{\Gamma}$ depend on the choice of the linear ordering of the set $E(\Gamma)$; however their product $c_{\Gamma} \Phi_{\Gamma}$ is independent of that choice.
} 
Proof. First we explain the condition $d-1 \mid n d-2$. If $\Gamma \in G_{n, l}$, then $c_{\Gamma} \neq 0$ if and only if $\Omega_{\Gamma}$ is a top degree differential form on $\bar{C}_{n}\left(\mathbb{R}^{d}\right)$, i.e. if and only if $\operatorname{deg} \Omega_{\Gamma}=(d-1) l$ is equal to $\operatorname{dim} \bar{C}_{n}\left(\mathbb{R}^{d}\right)=d n-d-1=d n-2-(d-1)$. Assume now that $n$ is such that $(d-1) \mid n d-3$. Then, for any $\Gamma \in G_{n, \frac{n d-3}{d-1}-1}$, one has, by the Stokes theorem,

$$
0=\int_{\bar{C}_{n}\left(\mathbb{R}^{d}\right)} d \Omega_{\Gamma}=\int_{\partial \bar{C}_{n}\left(\mathbb{R}^{d}\right)} \Omega_{\Gamma}=\sum_{\substack{A \subseteq[n] \\ \# A \geq 2}}(-1)^{\sigma_{A}} \int_{\bar{C}_{(n-A) \sqcup \bullet}\left(\mathbb{R}^{d}\right)} \Omega_{\Gamma / \Gamma_{A}} \int_{\bar{C}_{A}\left(\mathbb{R}^{d}\right)} \Omega_{\Gamma_{A}}=\sum_{\substack{A \subset V(\Gamma) \\ A \text { is admissible }}}(-1)^{\sigma(A)} c_{\Gamma_{A}} c_{\Gamma / \Gamma_{A}} .
$$

where a subset $A \subset V(\Gamma)$ is called admissible if $d-1 \mid d \# A-2$ and $\Gamma_{A} \in G_{\# A, \frac{d \# A-2}{d-1}-1}$. For admissible subsets $A \subset[n]$ both differential forms $\Omega_{\Gamma_{A}}$ and $\Omega_{\Gamma / \Gamma_{A}}$ are top degree forms on $\bar{C}_{\# A}\left(\mathbb{R}^{d}\right)$ and, respectively, $\bar{C}_{n-\# A+1}\left(\mathbb{R}^{d}\right)$. For a pair of natural number $m$ and $d$ such that $d-1 \mid m d-2$ let us denote

$$
\Gamma_{[m ; d]}:=G_{m, \frac{m d-2}{d-1}-1} .
$$

Then, using (37), we obtain,

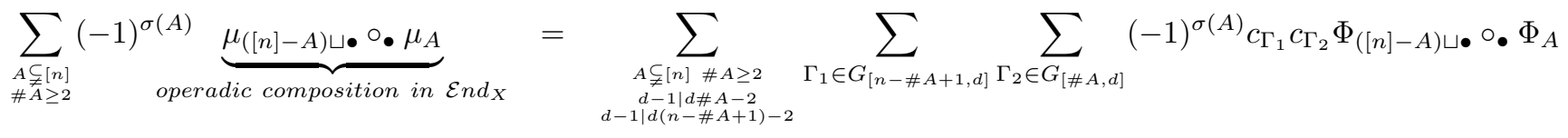

$$
\begin{aligned}
& =\sum_{\Gamma \in G_{n, \frac{n d-3}{d-1}-1}}\left(\sum_{\substack{A \subset V \text { ert }(\Gamma) \\
\text { A is admissible }}}(-1)^{\sigma_{A}} c_{\Gamma_{A}} c_{\Gamma / \Gamma_{A}}\right) \Phi_{\Gamma} \\
& =0
\end{aligned}
$$

which proves the claim.

8.2.4. Remark. The above proof is elementary but notationally looks unduly complicated — we have to take care about divisibility conditions of the type $d-1 \mid n d-2$ to ensure that the integrals $\int_{\bar{C}_{n}\left(\mathbb{R}^{d}\right)} \Omega_{\Gamma}$ make sense. If, however, we set formally $\int_{\bar{C}_{n}\left(\mathbb{R}^{d}\right)} \Omega_{\Gamma}=0$ when $\operatorname{deg} \Omega_{\Gamma} \neq \operatorname{dim} \bar{C}_{n}\left(\mathbb{R}^{d}\right)$, then the presentation gets simplified. We assume this convention from now on.

8.2.5. Example. For any $i, j \in[1, \ldots, n]$ there is a natural map

$$
\begin{aligned}
& \pi_{i j}: \quad C_{n}\left(\mathbb{R}^{d}\right) \quad \longrightarrow C_{2}\left(\mathbb{R}^{d}\right) \simeq S^{d-1} \\
& \left(x_{1}, \ldots, x_{n}\right) \quad \longrightarrow \quad \frac{x_{i}-x_{j}}{\left|x_{i}-x_{j}\right|}
\end{aligned}
$$

which forgets all points in the configuration except those labelled by $i$ and $j$. This map extends to a map of the compactifications, $\bar{\pi}_{i j}: \bar{C}_{n}\left(\mathbb{R}^{d}\right) \longrightarrow \bar{C}_{n}\left(\mathbb{R}^{d}\right)$.

8.2.5.1. Theorem. Let $\omega$ be a volume form on $S^{d-1}$ normalized so that $\int_{S^{d-1}} \omega=1$. Then the maps, $n \geq 2$,

$$
\begin{aligned}
\Omega: \mathfrak{G}(n) & \longrightarrow \\
\Gamma & \longrightarrow \Omega_{\Gamma}:=\bigwedge_{e \in E(\Gamma)} \omega_{\bar{C}_{n}\left(\mathbb{R}^{d}\right)} \omega_{e}
\end{aligned}
$$

define a de Rham field theory on $\bar{C}\left(\mathbb{R}^{d}\right)$. Here, for an edge e beginning at a vertex labelled by $i \in[n]$ and ending at a vertex labelled by $j \in[n]$, we set $\omega_{e}:=\bar{\pi}_{i j}^{*}(\omega)$.

Proof. Consider the boundary stratum in $\bar{C}\left(\mathbb{R}^{d}\right)$ corresponding to a graph,

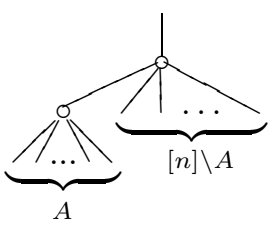

It is equal to the image of the map (41). Introduce in the neighbourhood of $\operatorname{Im}\left(\circ_{A}\right)$ a coordinate chart,

$$
\mathcal{U}_{A}=[0, \delta) \times C_{A}^{s t}\left(\mathbb{R}^{d}\right) \times C_{[n]-A+\bullet}^{s t}\left(\mathbb{R}^{d}\right),
$$


corresponding to the metric version of the above graph (see 8.3 ). The boundary stratum $\operatorname{Im}\left(\circ_{A}\right)$ is given in this chart by the equation $s=0$, where $s$ is the standard coordinate on the semi-open interval $[0, \delta)$ for some small $\delta \in \mathbb{R}^{+}$. Thus, to prove the Proposition, we have to check that

$$
\left.\Omega_{\Gamma}\right|_{s=0}=(-1)^{\varepsilon} p_{1}^{*}\left(\bigwedge_{e^{\prime} \in E\left(\Gamma / \Gamma_{A}\right)} \omega_{e^{\prime}}\right) \wedge p_{2}^{*}\left(\bigwedge_{e^{\prime \prime} \in E\left(\Gamma_{A}\right)} \omega_{e^{\prime \prime}}\right)
$$

where $p_{1}: C_{A}^{s t}\left(\mathbb{R}^{d}\right) \times C_{[n]-A+\bullet}^{s t}\left(\mathbb{R}^{d}\right) \rightarrow C_{[n]-A+\bullet}^{s t}\left(\mathbb{R}^{d}\right)$ and $p_{2}: C_{A}^{s t}\left(\mathbb{R}^{d}\right) \times C_{[n]-A+\bullet}^{s t}\left(\mathbb{R}^{d}\right) \rightarrow C_{A}^{s t}\left(\mathbb{R}^{d}\right)$ are natural projections, and the sign $(-1)^{\varepsilon}$ is given just by regrouping of the factors $\omega_{e}$ in $\Omega_{\Gamma}$, i.e. by the equality

$$
\Omega_{\Gamma}=(-1)^{\varepsilon}\left(\bigwedge_{e^{\prime} \in E(\Gamma) \backslash E\left(\Gamma_{A}\right)} \omega_{e^{\prime}}\right) \wedge\left(\bigwedge_{e^{\prime \prime} \in E\left(\Gamma_{A}\right)} \omega_{e^{\prime \prime}}\right)
$$

All points parameterized by $A$ collapse in the limit $s \rightarrow 0$ into a single point $x_{0} \in \mathbb{R}^{d}$; we can represent this limit configuration as $s \rightarrow 0$ limit of a configuration $\left\{x_{0}+s x_{i}\right\}_{i \in A} \sqcup\left\{x_{j}\right\}_{j \in[n] \backslash A}$. Using now invariance of the forgetful map $\pi_{i j}$ under the transformation $\left(x_{i} \rightarrow x_{0}+s x_{i}, x_{j} \rightarrow x_{0}+s x_{j}\right)$, we immediately conclude that

$$
\left.\bigwedge_{e^{\prime} \in E(\Gamma) \backslash E\left(\Gamma_{A}\right)} \omega_{e^{\prime}}\right|_{s=0}=p_{1}^{*}\left(\bigwedge_{e^{\prime} \in E\left(\Gamma / \Gamma_{A}\right)} \omega_{e^{\prime}}\right) \text { and }\left.\bigwedge_{e^{\prime \prime} \in E\left(\Gamma_{A}\right)} \omega_{e^{\prime}}\right|_{s=0}=p_{2}^{*}\left(\bigwedge_{e^{\prime \prime} \in E\left(\Gamma_{A}\right)} \omega_{e^{\prime \prime}}\right)
$$

as required.

For any vector space $V$ the associated vector space $\mathfrak{g}_{d}(V)$ is a representation of the operad $\mathfrak{G}$. Hence we can apply Theorem $\mathbf{8 . 2 . 3}$ and obtain the following

8.2.5.1. Corollary. The $\mathcal{L}_{\infty}$-structure, $\left\{\mu_{n}=\sum_{\Gamma \in \mathfrak{G}_{d}(n)} c_{\Gamma} \Phi_{\Gamma}\right\}_{n \geq 2}$, induced on $\mathfrak{g}_{d}(V)$ by the de Rham field theory (45) is homotopy non-trivial. For $\omega=\operatorname{Vol}\left(S^{d-1}\right)$, the standard homogeneous volume form on the sphere, the induced $\mathcal{L}_{\infty}$-structure is precisely the Poisson-Schouten structure (39).

Proof. Let us first consider the case $\omega=\operatorname{Vol}\left(S^{d-1}\right)$. Proof of the last sentence in the Corollary is based on two Kontsevich's vanishing lemmas. It was shown in $\mathrm{Ko}$ ] (for the case $d=2$ ) and in $\mathrm{Ko1}$ ] (for $d \geq 3$ ) that, for any integer $n \geq 3$ and for any graph $\Gamma \in \mathcal{G}_{d}(n)$, one has

$$
\int_{\bar{C}_{n}\left(\mathbb{R}^{d}\right)} \Omega_{\Gamma}=0
$$

Hence, for $n \geq 3$,

$$
\mu_{n}=\sum_{\Gamma \in \mathfrak{G}_{d}(n)} c_{\Gamma} \Phi_{\Gamma}=0
$$

and

$$
\begin{aligned}
\mu_{2} & =\sum_{\Gamma \in \mathfrak{G}_{d}((2))} c_{\Gamma} \Phi_{\Gamma} \\
& =c_{\Gamma_{1}} \Phi_{\Gamma_{1}}+c_{\Gamma_{2}} \Phi_{\Gamma_{2}} \\
& =\{\bullet\}_{-1},
\end{aligned}
$$

where we denoted $\Gamma_{1}=\stackrel{1}{\longrightarrow} \stackrel{2}{\longrightarrow}$ and $\Gamma_{2}=\stackrel{2}{\longrightarrow} \stackrel{1}{\longrightarrow}$ and used the fact that, by the normalization assumption on $\omega$, $c_{\Gamma_{1}}=c_{\Gamma_{2}}=1$.

A generic cohomologically non-trivial and normalized $(d-1)$-form $\omega$ on $S^{d-1}$ is given by

$$
\omega=\operatorname{Vol}\left(S^{d-1}\right)+d f
$$

for some semialgebraic function $f$ on $S^{d-1}$. In general, Kontsevich's vanishing lemmas are not true for nonhomogeneous propagators $\omega$, and one obtains a non-trivial (but homotopy trivial) $\mathcal{L}_{\infty}$-deformation of the PoissonSchouten bracket (see [Me2] for an explicit example). This fact implies that the resulting $L_{\infty}$-structure is never homotopy equivalent to the trivial (i.e. vanishing) $\mathcal{L}_{\infty}$-structure on $\mathfrak{g}_{d}(V)$. 
8.3. De Rham field theories on a a class of free topological operads. The above results for $\bar{C}\left(\mathbb{R}^{d}\right)$ have obvious analogues for all operads of configuration spaces considered in this paper. In fact, they hold true for any (coloured) operad $\bar{C}=\left\{\bar{C}_{n}\right\}_{n \geq 1}$ in the category of smooth manifolds with corners (or semialgebraic manifolds) which, as an operad in the category of sets, is free, $\bar{C}=\mathcal{F}$ ree $\langle C\rangle$, and satisfies the following conditions:

(i) each $\bar{C}_{n}$ is a compact oriented (semialgebraic) manifold;

(ii) the $\mathbb{S}$-space, $C=\left\{C_{p}\right\}_{p \geq 1}$, of generators is given by

$$
C_{p}:=\bar{C}_{p} \backslash \partial \bar{C}_{p}
$$

where $\partial \bar{C}_{p}$ is the boundary of $\bar{C}_{n}$;

(iii) as $\bar{C}=\mathcal{F}$ ree $\langle C\rangle$, each manifold $\bar{C}_{n}$ is canonically stratified, $\bar{C}_{n}=\coprod_{T \in \mathcal{T}_{n}} C_{T}$, into a disjoint union of cartesian products, $C_{T}=\prod_{v \in V(T)} C_{\# \operatorname{In}(v)}$. It is assumed that the set theoretic inclusion, $C_{T} \hookrightarrow \bar{C}_{n}$, is smooth (or semialgebraic) for any $T$.

It follows from these assumptions that the operadic composition in $\bar{C}$ corresponding to a tree $T \in \mathcal{T}_{n}$,

$$
\mu_{T}: T\langle\bar{C}\rangle \longrightarrow \bar{C}_{n}
$$

is a smooth map which sends the Cartesian product $T\langle\bar{C}\rangle \simeq \prod_{v \in V(T)} \bar{C}_{\# I n(v)}$ into a boundary stratum in $\bar{C}_{n}$. We define $(-1)^{\mu_{T}}$ to be +1 if this maps preserves orientations and $(-1)$ otherwise. We also have,

$$
\partial \bar{C}_{n}=\coprod_{T \in \mathcal{T}_{n}^{\text {boundary }}} \mu_{T}(T\langle\bar{C}\rangle)
$$

for a suitable subfamily $\mathcal{T}_{n}^{\text {boundary }} \subset \mathcal{T}_{n}$. For concreteness and simplicity, we assume from now on that $\bar{C}=\left\{\bar{C}_{n}\right\}_{n \geq 1}$ is one of the operads of compactified configuration spaces considered in $\S 2-4$ so that each connected component of the generator $C_{n}$ of $\bar{C}$ is a quotient of a configuration space, $\operatorname{Conf}_{n}(\mathbb{V})$, of pairwise distinct $[n]$-labeled points in a (subspace of a) vector space $\mathbb{V}$ modulo an action of an appropriate subgroup of $A f f(\mathbb{V})$.

Let $\overline{\mathcal{C}}_{n}$ be the vector space spanned by all possible pairs, $\left(\bar{C}_{n}^{o}\right.$,or $)$, consisting of a connected component, $\bar{C}_{n}^{o}$, of $\bar{C}_{n}$ and a choice of orientation, or, on $\bar{C}_{n}^{o}$ modulo an equivalence relation, $\left(\bar{C}_{n}^{o},-o r\right)=-\left(\bar{C}_{n}^{o}\right.$,or $)$. The standard orientation on $\bar{C}_{n}^{o}$ is denoted by $o r^{0}$. The collection $\langle\overline{\mathcal{C}}\rangle:=\left\{\left\langle\overline{\mathcal{C}}_{n}\right\rangle\right\}_{n \geq 1}$ is naturally an $\mathbb{S}$-module. The operad of fundamental chains or the face complex of $\bar{C}$ i: 21 , by definition, a dg free operad, $\mathcal{F C h a i n s}(\bar{C}):=(\mathcal{F}$ ree $\langle\overline{\mathcal{C}}\rangle, \partial)$ whose differential is read off from the above formula for $\partial \bar{C}_{n}$,

$$
\partial\left(\bar{C}_{n}^{o}, \text { or }^{o}\right)=\sum_{T \in \mathcal{T}_{n}^{\text {boundary }}}(-1)^{\mu_{T}} T\left\langle\left(\bar{C}_{\bullet}^{o}, \text { or }^{o}\right)\right\rangle .
$$

(see explicit formulae given in $\S 2-4$ for particular examples).

8.3.1. Definition-Theorem. Let $\mathfrak{G}^{\circlearrowright}$ be an arbitrary (coloured) operad of Feynman graphs. A de Rham field theory (of type $\mathfrak{G}^{\circlearrowright}$ ) on a semialgebraic (coloured) operad $\bar{C}=\left\{\bar{C}_{n}\right\}_{n \geq 1}$ is a morphism, $\Omega:\left(\mathfrak{\mathfrak { G }}^{\circlearrowright}, 0\right) \longrightarrow\left(\Omega_{\bar{C}}, d_{D R}\right)$, of $d g$ cooperads. Let

$$
\begin{aligned}
& \rho: \mathfrak{G}^{\circlearrowright} \longrightarrow \mathcal{E} n d_{X} \\
& \Gamma \longrightarrow \Phi_{\Gamma}
\end{aligned}
$$

be a representation of the operad of Feynman graphs in a graded vector space $X$ (or in a family of vector spaces parameterized by the set of colours). Then a de Rham field theory, $\Omega$, on $\bar{C}=\left\{\bar{C}_{n}\right\}$ makes the graded vector space $X$ into a representation,

$$
\begin{aligned}
& \rho_{Q}: \quad \mathcal{F C h a i n s}(\bar{C}) \longrightarrow \quad \mathcal{E} n d_{X} \\
& \left(\bar{C}_{n}^{o},{ }^{o}{ }^{o}\right) \quad \longrightarrow \quad \mu_{n} \in \operatorname{Hom}\left(X^{\otimes n}, X\right)
\end{aligned}
$$

of the operad of fundamental chains on $\bar{C}$ given explicitly by

$$
\mu_{n}:=\sum_{\Gamma \in \mathfrak{G}(n)}\left(\int_{\bar{C}_{n}^{o}} \Omega(\Gamma)\right) \Phi_{\Gamma} .
$$

The representation $\rho_{Q}$ is called a quantization of the representation $\rho$.

\footnotetext{
${ }^{21}$ The most natural way to define this notion is to use the theory of semialgebraic chains developed in [KS, HLTV].
} 
Proof. The natural pairing,

$$
\begin{aligned}
& \text { Chains }(\bar{C}) \times \Omega(\bar{C}) \longrightarrow \mathbb{C} \\
& \sigma \quad \omega \longrightarrow \int_{\sigma} \omega
\end{aligned}
$$

gives rise to a morphisms of operads,

$$
\begin{array}{ccc}
\Omega^{*}: \begin{array}{c}
\text { Chains }(\bar{C}) \\
\sigma
\end{array} & \longrightarrow \sum_{\Gamma}\left(\int_{\sigma} \Omega_{\Gamma}\right) \Gamma
\end{array}
$$

The required representation $\rho_{Q}$ is given finally by the composition,

$$
\mathcal{F C h a i n s}(\bar{C}) \hookrightarrow \mathcal{C} \text { hains }(\bar{C}) \stackrel{\Omega^{*}}{\longrightarrow} \mathfrak{G}^{\circlearrowright} \stackrel{\rho}{\longrightarrow} \mathcal{E} n d_{X}
$$

8.3.2. Important remark. In down to earth terms a de Rham field theory on $\bar{C}$ is a family of maps

$$
\left\{\begin{array}{ccc}
\Omega: G_{n} & \longrightarrow & \Omega_{\text {closed }}^{\bullet}\left(\bar{C}_{n}\right) \\
\Gamma & \longrightarrow & \Omega_{\Gamma}
\end{array}\right\}_{n \geq 1, l \geq 0}
$$

such that, for any boundary stratum in $\bar{C}_{n}\left(\mathbb{R}^{d}\right)$ given by the image of a reduced operadic composition or, respectively, a "full" composition one has

$$
\circ_{A}^{*}\left(\Omega_{\Gamma}\right)=(-1)^{\varepsilon} \Omega_{\Gamma / \Gamma_{A}} \wedge \Omega_{\Gamma_{A}}
$$

or, respectively,

$$
\circ_{I_{1} \sqcup \ldots \sqcup I_{p}}^{*}\left(\Omega_{\Gamma}\right)=(-1)^{\varepsilon} \Omega_{\Gamma /\left\{\Gamma_{I_{1}}, \ldots, \Gamma_{I_{p}}\right\}} \wedge \Omega_{\Gamma_{I_{1}}} \wedge \Omega_{\Gamma_{I_{2}}} \wedge \ldots \wedge \Omega_{\Gamma_{I_{p}}}
$$

where the signs are determined, as usually, by comparison of orientations on the sets of edges. Once the above conditions hold true, formulae (47) define a quantization of an arbitrary representation of an operad of Feynman diagrams. Here $\Omega_{\text {closed }}^{\bullet}\left(\bar{C}_{n}\right) \subset \Omega^{\bullet}\left(\bar{C}_{n}\right)$ stands for the subspace of closed differential forms.

We are interested in this paper only in deformation quantization of representations of the suboperad of $\mathcal{C}$ hains $(\bar{C})$ spanned by the fundamental chains. As it stands, Theorem 8.3.1 holds true for representations of the full chain operad as well; for this Theorem to work only at the level of fundamental chains only one can weaken the notion of a de Rham field theory by requiring that factorization properties (48)-(49) hold only for top degree differential forms on $\bar{C}$. From now on we understand by a de Rham field theory on $\bar{C}$ such a weakened version as well.

\section{Examples of quantized representations of operads of Feynman diagrams}

9.1. Propagators on $\bar{C}\left(\mathbb{H}^{d}\right)$. Let us consider the operad (see 44.3 )

$$
\bar{C}\left(\mathbb{H}^{d}\right)=\bar{C} \cdot\left(\mathbb{R}^{d}\right) \coprod \bar{C}_{\bullet}, \bullet\left(\mathbb{H}^{d}\right)
$$

for $d \geq 2$. The space $\bar{C}_{2,0}\left(\mathbb{H}^{d}\right)$ is a compact $d$-dimensional semialgebraic space of the form

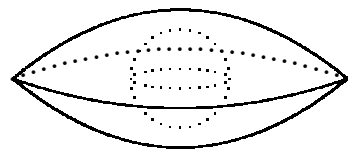

Its boundary is a union $S_{i n}^{d-1} \cup S_{+}^{d-1} \cup S_{-}^{d-1}$, where $S_{i n}^{d-1}$ is the sphere corresponding to two points in the upper half space $\mathbb{H}^{d}$ collapsing to a point in $\mathbb{H}^{d}, S_{+}^{d-1}$ (respectively, $S_{+}^{d-1}$ ) is the half-sphere corresponding to the limit configurations where the point labelled 1 (respectively, 2) approaches the boundary, $\partial \overline{\mathbb{H}}^{d}$, of the closed upper-half space.

The space $\bar{C}_{2,0}\left(\mathbb{H}^{d}\right)$ is homotopy equivalent to $S^{d-1}$. Any cohomologically non-trivial $P A$-form $\omega$ on $\bar{C}_{2,0}\left(\mathbb{H}^{d}\right)$ normalized so that

$$
\int_{S_{i n}^{d-1}} \omega^{i n}=1, \quad \text { where } \omega^{i n}:=\left.\omega\right|_{S_{i n}^{d-1}}
$$


defines a de Rham field theory of type $\mathfrak{G}^{\uparrow \downarrow}$ on the 2-coloured operad $\bar{C}\left(\mathbb{H}^{d}\right)$ by the following two sets of maps (see Remark 8.3.2)

$$
\left\{\begin{array}{ccc}
\Omega_{i n}: \mathfrak{G}(n) & \longrightarrow & \Omega^{(d-1) \# E(\Gamma)}\left(\bar{C}_{n}\left(\mathbb{R}^{d}\right)\right) \\
\Gamma & \longrightarrow & \Omega_{i n}(\Gamma):=\bigwedge_{e \in E(\Gamma)} p_{e}^{*}\left(\omega^{i n}\right)
\end{array}\right\}
$$

and

$$
\left\{\begin{array}{ccc}
\Omega: \mathrm{G}^{\uparrow \downarrow}(n+m) & \longrightarrow & \Omega^{(d-1) \# E(\Gamma)}\left(\bar{C}_{n, m}\left(\mathbb{H}^{d}\right)\right) \\
\Gamma & \longrightarrow & \Omega(\Gamma):=\bigwedge_{e \in E(\Gamma)} \pi_{e}^{*}(\omega)
\end{array}\right\}
$$

Here

$$
p_{e}: \bar{C}_{n}\left(\mathbb{R}^{d}\right) \longrightarrow \bar{C}_{2}\left(\mathbb{R}^{d}\right), \quad \text { and } \quad \pi_{e}: C_{n, m}\left(\mathbb{H}^{d}\right) \longrightarrow \bar{C}_{2,0}\left(\mathbb{R}^{d}\right)
$$

are the natural forgetful maps. To prove this claim we have to check factorization property (48) for the boundary stratum in $\bar{C}_{n, m}$ corresponding to graphs of the form,

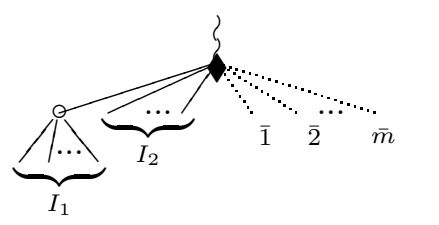

and

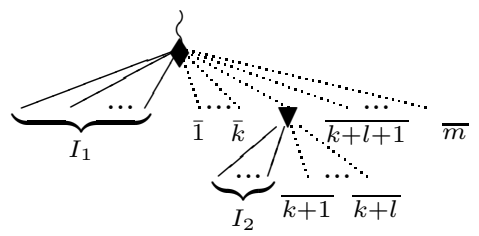

These strata are images of the following operadic compositions,

$$
\circ_{I_{1}}: \bar{C}_{\# I_{2}+1, m}\left(\mathbb{H}^{d}\right) \times \bar{C}_{I_{1}}\left(\mathbb{R}^{d}\right) \rightarrow \bar{C}_{n, m}\left(\mathbb{H}^{d}\right) \quad \text { and } \quad \circ \overline{k+1}: \bar{C}_{\# I_{1}, m-l+1}\left(\mathbb{H}^{d}\right) \times \bar{C}_{\# I_{1}, l}\left(\mathbb{H}^{d}\right) \rightarrow \bar{C}_{n, m}\left(\mathbb{H}^{d}\right),
$$

so that the required factorization conditions are given, respectively, by

$$
\circ_{I_{1}}^{*}(\Omega(\Gamma))=(-1)^{\epsilon} \Omega\left(\Gamma / \Gamma_{I_{1}}\right) \wedge \Omega_{\text {in }}\left(\Gamma_{I_{1}}\right) \quad \text { and } \quad \circ^{*}(\Omega(\Gamma))=(-1)^{\varepsilon} \Omega\left(\Gamma / \Gamma_{A}\right) \wedge \Omega\left(\Gamma_{A}\right)
$$

where we set $A:=I_{2} \sqcup\{\overline{k+1}, \ldots, \overline{k+l}\}$. Both these conditions can be checked in the associated coordinate charts,

$$
\mathcal{U}_{1}=[0, \delta) \times C_{\# I_{2}+1, m}^{s t}\left(\mathbb{H}^{d}\right) \times C_{\# I_{1}}\left(\mathbb{R}^{d}\right) \text { and } \mathcal{U}_{1}=[0, \delta) \times C_{\# I_{1}+1, m-l}^{s t}\left(\mathbb{H}^{d}\right) \times C_{\# I_{1}, l}\left(\mathbb{H}^{d}\right)
$$

using the same arguments as in in the proof of Theorem 8.2.5.1 but with two small subtleties:

(1) in the limit $s \rightarrow 0, s \in[0, \delta)$, the differential form $\wedge_{e^{\prime \prime} \in E\left(\Gamma_{I_{1}}\right)} \omega_{e}$ does not stay invariant but tends to $\wedge_{e^{\prime \prime} \in E\left(\Gamma_{I_{1}}\right)} \omega_{e}^{i n}$ explaining thereby appearance of $\Omega_{i n}$ in the r.h.s. of the first factorization condition;

(2) contrary to the above case (1) the differential form $\wedge_{e^{\prime \prime} \in E\left(\Gamma_{A}\right)} \omega_{e}$ in the limit $s \rightarrow 0$ does stay invariant; the reason is that the point into which $A$-labelled points collapse is located on the boundary of the upper-half space and, with no loss of generality, can be placed at $0 \in \overline{\mathbb{H}^{d}}$; then, by definition of the coordinate chart $\mathcal{U}_{2}$, the parameter $s$ acts on the $A$-labelled configuration as an ordinary dilation while each $\omega_{e^{\prime \prime}}$ is both translation- (along $\mathbb{R}^{d-1} \subset \overline{\mathbb{H}^{d}}$ ) and dilation-invariant.

Therefore, we have the following

9.1.1. Theorem. Given a representation, $\rho: \mathfrak{G}^{\uparrow \uparrow} \rightarrow \mathcal{E} n d_{V_{c}, V_{o}}$, of the 2-coloured operad of Feynman diagrams in a pair of vector spaces $\left(V_{c}, V_{o}\right)$. Then, for any any homologically non-trivial smooth (or PA) differential $(d-1)$-form $\omega$ on $\bar{C}_{2,0}\left(\mathbb{H}^{d}\right)$, the formulae

$$
\nu_{n}:=\sum_{\Gamma \in G_{n}}\left(\int_{\bar{C}_{n}\left(\mathbb{R}^{d}\right)} \Omega_{i n}(\Gamma)\right) \rho(\Gamma)
$$

define a $L_{\infty}\{d-1\}$-structure on the vector space $V_{c}$, and, for any $M C$-element $\gamma$ in $\left(V_{c}, \nu_{\bullet}\right)$, the formulae,

$$
\mu_{m}^{\gamma}\left(v_{1}, \ldots, v_{m}\right):=\sum_{n=0}^{\infty} \frac{\hbar^{n}}{n !} \sum_{\Gamma \in G_{n+m}}\left(\int_{\bar{C}_{n, m}\left(\mathbb{H}^{d}\right)} \Omega(\Gamma)\right) \rho(\Gamma)\left(\gamma^{\otimes n} \otimes v_{1} \otimes \ldots \otimes v_{m}\right), \quad v_{i} \in V_{0},
$$

define,

(i) in general, a non-flat $A_{\infty}$-algebra structure in $V_{o}[[\hbar]]$ for $d=2$,

(ii) in general, a non-flat $L_{\infty}\{d-1\}$-algebra structure in $V_{o}[[\hbar]]$ for $d \geq 3$.

\subsection{Examples of propagators.}


9.2.1. Homogeneous volume form on a sphere. A differential form on $C_{2,0}\left(\mathbb{H}^{d}\right)$,

$$
\omega_{0}:=p^{*}\left(\operatorname{Vol}\left(S^{d-1}\right)\right)
$$

where $p: C_{2,0}\left(\mathbb{H}^{d}\right) \rightarrow C_{2}\left(\mathbb{R}^{d}\right)$ is the natural projection, extends to the compactification $\bar{C}_{2,0}\left(\mathbb{H}^{d}\right)$ and hence defines a non-trivial de Rham field theory on the operad $\bar{C}\left(\mathbb{H}^{d}\right)$.

9.2.2. Kontsevich (anti)propagators. Let $\left[z_{1}, z_{2}\right]$ be an arbitrary configuration in $C_{2,0}\left(\mathbb{H}^{d}\right)$ and $\left(z_{1}, z_{2}\right)$ be its arbitrary representative in $\operatorname{Conf}_{2,0}\left(\mathbb{H}^{d}\right)$. Let

$$
d s^{2}:=\frac{d x_{1}^{2}+\ldots+d x_{d}^{2}}{x_{d}^{2}}
$$

be the standard hyperbolic metric on $\mathbb{H}^{d}:=\left\{\left(x_{1}, \ldots, x_{d}\right) \in \mathbb{R}^{d} \mid x_{d}>0\right\}$. Let $S_{H}^{d-1}\left(z_{1}\right)$ and $\omega_{K}^{0}$ be the unit hyperbolic sphere centered at $z_{1}$ and, respectively, its induced normalized volume form (with respect to the above metric). Using the unique hyperbolic geodesic $g\left(z_{1}, z_{2}\right)$ from $z_{1}$ to $z_{2}$,

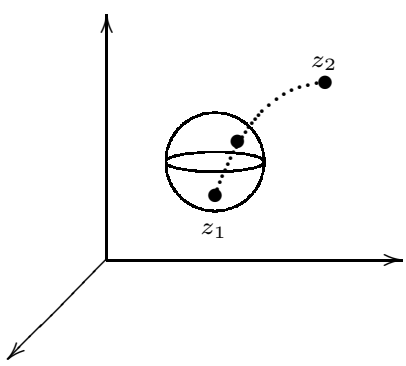

we define a smooth map

$$
\begin{array}{rlc}
p_{H}: \operatorname{Conf}_{2,0}\left(\mathbb{H}^{d}\right) & \longrightarrow & S_{H}^{d-1}\left(z_{1}\right) \\
\left(z_{1}, z_{2}\right) & \longrightarrow g\left(z_{1}, z_{2}\right) \cap S_{H}^{d-1}\left(z_{1}\right)
\end{array}
$$

and set

$$
\omega_{K}\left(z_{1}, z_{2}\right):=p_{H}^{*}\left(\omega_{K}^{0}\right)
$$

This form is $\mathbb{R}^{*} \ltimes \mathbb{R}^{d}$-invariant and hence defines a closed homologically non-trivial $(d-1)$-form on $C_{2,0}\left(\mathbb{H}^{d}\right)$. Moreover, it extends to the compactification $\bar{C}_{2,0}\left(\mathbb{H}^{d}\right)$. For $d=2$ this form is precisely the Kontsevich propagator used in the construction of his formality map [Ko2]; hence the notation. We set $\omega_{\bar{K}}\left(z_{1}, z_{2}\right):=\omega_{K}\left(z_{2}, z_{1}\right)$ and call it Kontsevich antipropagator.

9.3. Deformation quantization of associative algebras of polyvector fields. For any graded vector space $V$, the associated pair $X_{c}=X_{o}=\mathfrak{g}_{d}(V)$ carries a natural representation of the Feynman operad $\mathfrak{G}^{\uparrow \downarrow}$. For propagators considered in $\$ \mathbf{9 . 2}$ the induced $L_{\infty}\{d-1\}$ algebra structure on $X_{c}$ is, by Corollary 98.2 .5 .1 precisely the Poisson-Schouten bracket (39). Hence any propagator from $\$ \mathbf{9 . 2}$ together with an MC element $\gamma$ in the degree $1-d$ Lie algebra $\left(\mathfrak{g}_{d}(V),\{\bullet\}\right)$ makes, by Theorem 9.1.1. $\mathfrak{g}_{d}(V)$ into a (non-flat) $A_{\infty}$-algebra for $d=2$ or into a $L_{\infty}\{d-1\}$-algebra for $d \geq 3$. The case $d \geq 3$ will be considered in more detail below while in this subsection we assume from now on that $d=2$ so that $\left(\mathfrak{g}_{2}(V),\{\bullet\}\right)$ is the Schouten algebra, $\mathcal{T}_{\text {poly }}(V)$, of formal polyvector fields on $V$. Then an arbitrary Poisson structure, $\gamma \in \mathcal{T}_{\text {poly }}(V)$, and any propagator from the set $\left\{\omega_{0}, \omega_{K}, \omega_{\bar{K}}\right\}$ defines by formulae (52) a non-flat $A_{\infty}$ structure on $\mathcal{T}_{\text {poly }}(V)$. For $\gamma=0$ the only graph contributing to (52) is the one consisting of two vertices on the real line with no edges,

so that $\mu_{m}^{\gamma=0}=0$ for $m \neq 2$ and $\mu_{2}^{\gamma=0}$ is the ordinary product of polyvector fields. Thus, for $\gamma \neq 0$, formulae (52) describe a deformation quantization of that ordinary product in $\mathcal{T}_{\text {poly }}(V)$. Using de Rham field theories discussed in $\$ \mathbf{1 0}$ below using a de Rham field theory on $\widehat{\mathfrak{C}}(\mathbb{H})$ one can show that the three non-flat $A_{\infty}$-algebras structures on $\mathcal{T}_{\text {poly }}(V)$ corresponding to propagators $\left\{\omega_{0}, \omega_{K}, \omega_{\bar{K}}\right\}$ are all homotopy equivalent to each other. The simplest of them is given by the propagator $\omega_{0}$ as in this case

$$
\int_{\bar{C}_{n, 0}(\mathbb{H}} \omega_{0}(\Gamma)=0
$$


for any graph $\Gamma \in \mathfrak{G}_{n, 2 n-2}$ with $n \geq 2$ (the reason is that the differential form $\omega_{0}(\Gamma)$ is invariant under vertical translations and hence vanishes for degree reasons). Hence the only graph contributing to $\mu_{0}^{\gamma}$ is the following one

so that $\mu_{0}^{\gamma}=\gamma$. The next term of the $A_{\infty}$-structure on $\mathcal{T}_{\text {poly }}(V)$ is given by,

$$
\mu_{1}=\sum_{n \geq 1} \sum_{\Gamma \in \mathfrak{G}_{n+1,2 n-1}} \int_{\bar{C}_{n, 1}} \Omega(\Gamma) \Phi_{\Gamma} .
$$

The initial $n=1$ term in this sum is given by the graph

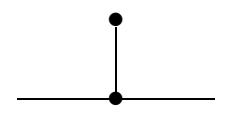

of weight 1 ; the associated operator $\Phi_{\Gamma}$ is $\{\gamma \bullet \ldots\}$. Using the reflection $z \rightarrow-\bar{z}$ one can easily check that $\int_{\bar{C}_{n, 1}} \Omega(\Gamma)=0$ for $n$ even. For a graph $\Gamma \in \mathfrak{G}_{n+1,2 n-1}$ let us denote by $\Gamma_{i}$ its drawing in $C_{n, 1}$ with the vertex labelled by $i$ put at the point in $\mathbb{R}$; for any $n \geq 2$ we have, by Kontsevich vanishing Lemma 6.4 in [Ko2],

$$
\sum_{i=1}^{n} \int_{\bar{C}_{n, 1}(\mathbb{H})} \Omega\left(\Gamma_{i}\right)=\int_{\bar{C}_{n+1}(\mathbb{C})} \Omega(\Gamma)=0
$$

so that

$$
\mu_{1}^{\gamma}\left(\mu_{0}^{\gamma}\right)=\mu_{1}^{\gamma}(\gamma)=\{\gamma \bullet \gamma\}=0
$$

which is in a full agreement with the claim that formulae (52) for $\omega=\omega_{0}$ define a non-flat $A_{\infty}$-structure. The graphs

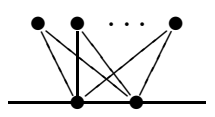

give, for example, non-zero contributions to $\mu_{2}^{\gamma}$ while

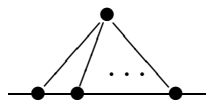

and their products contribute to higher homotopies, $\mu_{m \geq 3}^{\gamma}$. These graphs are not, of course, the only non-trivial contributions; for example, the weight of the following graph

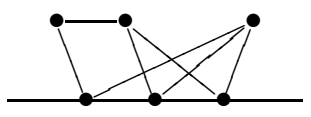

is non-zero so that it contributes, in general, to $\mu_{3}^{\gamma}$. As we shall see below, this non-flat $A_{\infty}$-structure on $\mathcal{T}_{\text {poly }}(V) \simeq$ $\odot \bullet V \otimes \wedge^{\bullet} V^{*}$ interpolates, in a sense, two Koszul dual deformation quantizations on $\odot{ }^{\bullet} V$ and $\wedge^{\bullet} V^{*}$ associated with propagators $\omega_{K}$ and, respectively, $\omega_{\bar{K}}$. Note in this connection that $\omega_{0}=\frac{1}{2}\left(\omega_{K}+\omega_{\bar{K}}\right)$.

9.4. Kontsevich's formality maps. As we saw in the previous section, any cohomologically non-trivial differential 1-form, $\omega$, on $\bar{C}_{2}(\mathbb{H})$ gives us a non-trivial $A_{\infty}$ structure on $\mathcal{T}_{\text {poly }}(V)$. The latter algebra contains two subalgebras,

$$
\left.\odot V^{*}\right)=: \mathcal{O}_{V} \quad \text { and } \quad \odot(V[-1])=: \mathcal{O}_{V^{*}[1]}
$$

which can be viewed as the rings of smooth formal function on affine manifolds $V$ and, respectively, $V^{*}[1]$. Note that the pairs,

$$
\left(X_{c}=\mathcal{T}_{\text {poly }}(V), X_{o}^{\prime}=\mathcal{O}_{V}\right) \quad \text { and } \quad\left(X_{c}=\mathcal{T}_{\text {poly }}(V), X_{o}^{\prime \prime}=\mathcal{O}_{V^{*}[1]}\right)
$$

carry naturally representations of Feynman operads $\mathfrak{G}^{\downarrow}$ and, respectively, $\mathfrak{G}^{\uparrow}$ (see $\$ \mathbf{7 . 3}$ ). It is an elementary exercise to check that any cohomologically nontrivial normalized differential 1-form $\omega$ on $C_{2,0}(\mathbb{H})$ satisfying the condition $\left.\omega\right|_{S_{+}^{1}=0}$ (respectively, $\left.\left.\omega\right|_{S_{-}^{1}}=0\right)$ defines by formulae (50) and (51) a De Rham field theory on $\bar{C}(\mathbb{H})$ of type $\mathfrak{G}^{\downarrow}$ (respectively, $\mathfrak{G}^{\uparrow}$ ). 
The Kontsevich propagator $\omega_{K}$ satisfies the condition $\left.\omega_{K}\right|_{S_{+}^{1}=0}$ and hence defines by formulae (52) a morphism of operads,

$$
\mathcal{F C h a i n s}\left(\bar{C}\left(\mathbb{H}^{d}\right) \longrightarrow \mathcal{E} n d_{\left\{X_{c}=\mathcal{T}_{\text {poly }}(V), X_{o}^{\prime}=\mathcal{O}_{V}\right\}}\right.
$$

which is the same as his famous formality map. Any MC element $\gamma$ in the Schouten algebra $\mathcal{T}_{\text {poly }}(V)$ makes $\mathcal{O}_{V}[[\hbar]]$ into a (non-flat, in general) $\mathcal{A}_{\infty}$-algebra. The same propagator $\omega_{K}$ and the same MC element $\gamma$ make also $\mathcal{T}_{\text {poly }}(V)[[\hbar]]$ into a (non-flat) $\mathcal{A}_{\infty}$-algebra. It is clear that the natural inclusion

$$
\mathcal{O}_{V}[[\hbar]] \longrightarrow \mathcal{T}_{\text {poly }}(V)[[\hbar]]
$$

is a morphism of these $\mathcal{A}_{\infty}$-algebras which we denote by $F_{K}:\left(\mathcal{O}_{V}[[\hbar]], \omega_{K}\right) \rightarrow\left(\mathcal{T}_{\text {poly }}(V)[[\hbar]], \omega_{K}\right)$, the symbol $\omega_{K}$ indicates the origin of the induced $\mathcal{A}_{\infty}$-structures.

Similarly, the Kontsevich antipropagator $\omega_{\bar{K}}$ satisfies the condition $\left.\omega_{K}\right|_{S_{-}^{1}=0}$ and hence defines a morphism of operads,

$$
\mathcal{F C h a i n s}\left(\bar{C}\left(\mathbb{H}^{d}\right) \longrightarrow \mathcal{E} n d_{\left\{X_{c}=\mathcal{T}_{\text {poly }}(V), X_{o}^{\prime \prime}=\mathcal{O}_{V^{*}[1]}\right\}}\right.
$$

which coincides again with Kontsevich's formality map. Thus any MC element $\gamma$ in the Schouten algebra $\mathcal{T}_{\text {poly }}(V)$ makes $\mathcal{O}_{V^{*}[1]}[[\hbar]]$ into a (non-flat) $\mathcal{A}_{\infty}$-algebra. The same antipropagator $\omega_{\bar{K}}$ and the same MC element $\gamma$ make also $\mathcal{T}_{\text {poly }}(V)[[\hbar]]$ into a (non-flat) $\mathcal{A}_{\infty}$-algebra. The natural inclusion

$$
\mathcal{O}_{V^{*}[1]}[[\hbar]] \longrightarrow \mathcal{T}_{\text {poly }}(V)[[\hbar]]
$$

is a morphism of $\mathcal{A}_{\infty}$-algebras which we denote by $F_{\bar{K}}:\left(\mathcal{O}_{V^{*}[1]}[[\hbar]], \omega_{\bar{K}}\right) \rightarrow\left(\mathcal{T}_{\text {poly }}(V)[[\hbar]], \omega_{\bar{K}}\right)$.

Using the 4-coloured operad $\widehat{\mathfrak{C}}(\mathbb{H})$ one can construct explicitly $\mathcal{A}_{\infty}$-quasi-isomorphisms (in any direction),

$$
\left(\mathcal{T}_{\text {poly }}(V)[[\hbar]], \omega_{K}\right) \rightleftarrows\left(\mathcal{T}_{\text {poly }}(V)[[\hbar]], \omega_{0}\right) \leftrightarrows\left(\mathcal{T}_{\text {poly }}(V)[[\hbar]], \omega_{\bar{K}}\right)
$$

and hence a diagram of canonical $\mathcal{A}_{\infty}$-morphisms,

$$
\left(\mathcal{O}_{V}[[\hbar]], \omega_{K}\right) \hookrightarrow\left(\mathcal{T}_{\text {poly }}(V)[[\hbar]], \omega_{K}\right) \rightleftarrows\left(\mathcal{T}_{\text {poly }}(V)[[\hbar]], \omega_{0}\right) \leftrightarrows\left(\mathcal{T}_{\text {poly }}(V)[[\hbar]], \omega_{\bar{K}}\right) \hookleftarrow\left(\mathcal{O}_{V^{*}[1]}[[\hbar]], \omega_{\bar{K}}\right)
$$

It would be interesting to see if this diagram can be used to define Koszul duality of generic non-flat $\mathcal{A}_{\infty}$-structures on $\mathcal{O}_{V}$ and $\mathcal{O}_{V^{*}[1]}$. For a subclass of MC elements $\gamma$ which make $\left(\mathcal{O}_{V}[[\hbar]], \omega_{K}\right)$ and $\left(\mathcal{O}_{V^{*}[1]}[[\hbar]], \omega_{\bar{K}}\right)$ into flat $\mathcal{A}_{\infty}$ algebras the Koszul duality was already established in [Sh2, CFFR] (in fact, not all $\mathcal{A}_{\infty}$ algebras appearing in CFFR must be flat).

9.5. Deformation quantization of the Schouten bracket. In this section the propagator degree, $d-1$, of all operads under consideration is set by default to 2 , i.e. $d=3$.

For any vector space $V$, consider the following pair of Schouten-Poisson algebras,

$$
\mathfrak{g}_{2}(V)=\left(\odot \bullet\left(V^{*} \oplus V[-1]\right), \quad\{\bullet\}_{-1}\right) \simeq \mathcal{T}_{\text {poly }}(V)
$$

and

$$
\mathfrak{g}_{3}\left(V^{*}[1] \oplus V\right)=\left(\odot \bullet\left(V^{*} \oplus V[-1] \oplus V[-2] \oplus V^{*}[-1]\right),\{,\}_{-2}\right) .
$$

If $\left\{x^{\alpha}\right\}$ is a basis in $V^{*}$, and $\left\{\psi_{\alpha}\right\},\left\{\eta^{\alpha}\right\}$ and $\left\{y_{\alpha}\right\}$ the associated bases in $V[-1], V^{*}[-1]$ and, respectively, $V[-2]$, then

$$
\mathfrak{g}_{2}(V) \simeq \mathbb{R}\left[\left[x^{\alpha}, \psi_{\alpha}\right]\right] \text { with }\{f \bullet g\}_{-1}:=\sum_{\alpha} \frac{f \overleftarrow{\partial}}{\partial \psi_{\alpha}} \frac{\vec{\partial} g}{\partial x^{\alpha}}+(-1)^{|f||g|+f+g} \frac{g \overleftarrow{\partial}}{\partial \psi_{\alpha}} \frac{\vec{\partial} f}{\partial x^{\alpha}}
$$

and

$$
\mathfrak{g}_{3}\left(V[1] \oplus V^{*}\right) \simeq \mathbb{R}\left[\left[x^{\alpha}, \psi_{\alpha}, \eta^{\alpha}, y_{\alpha}\right]\right]
$$

with

$$
\{f, g\}_{-2}:=\sum_{\alpha}\left(\frac{f \overleftarrow{\partial}}{\partial y_{\alpha}} \frac{\vec{\partial} g}{\partial x^{\alpha}}+\frac{f \overleftarrow{\partial}}{\partial \eta^{\alpha}} \frac{\vec{\partial} g}{\partial \psi_{\alpha}}-(-1)^{|f||g|} \frac{g \overleftarrow{\partial}}{\partial y_{\alpha}} \frac{\vec{\partial} f}{\partial x^{\alpha}}-(-1)^{|f||g|} \frac{g \overleftarrow{\partial}}{\partial \eta^{\alpha}} \frac{\vec{\partial} f}{\partial \psi_{\alpha}}\right)
$$

where we use the fact that $\left|\psi_{a}\right|=-\left|x^{a}\right|+1,\left|\eta^{a}\right|=\left|x^{a}\right|+1,\left|y_{a}\right|=-\left|x^{a}\right|+2$. Note that $\delta:=\sum_{a} \eta^{a} y_{a}$ is an MC element in $\left(\mathfrak{g}_{3}\left(V[1] \oplus V^{*}\right),\{,\}_{-2}\right)$ making the latter into a $d g$ Lie algebra with the de Rham type differential $d:=\{\delta,\}_{-2} ;$ its cohomology is equal to $\mathbb{R}$.

There is a natural projection

$$
\pi_{\downarrow}: \mathfrak{g}_{3}\left(V^{*}[1] \oplus V\right) \rightarrow \mathfrak{g}_{2}(V)
$$


so that formulae completely analogous to (38) and (40) make the pair

$$
X_{1}=\mathfrak{g}_{3}\left(V[1] \oplus V^{*}\right) \quad \text { and } \quad X_{2}=\mathcal{T}_{\text {poly }}(V)
$$

into a representation of the 2-coloured operad $\mathfrak{G}^{\downarrow}$, i.e. there exists a canonical morphism of 2-coloured operads,

$$
\rho: \mathfrak{G}^{\downarrow} \longrightarrow \mathcal{E} n d_{X_{1}, X_{2}} \text {. }
$$

On the other hand, the propagator $\omega_{K}$ on $\bar{C}_{2,0}\left(\mathbb{H}^{3}\right)$ defines, by Theorem $\mathbf{8 . 3 . 1}$, a morphism of 2-coloured operads,

$$
\begin{array}{cl}
\mathcal{O C} \mathcal{L}_{\infty}:=\mathcal{F C h a i n s}\left(\bar{C}\left(\mathbb{H}^{3}\right)\right) & \longrightarrow \\
\sigma & \longrightarrow \sum_{\Gamma}\left(\int_{\sigma} \Omega_{\Gamma}\right) \Gamma
\end{array}
$$

so that the composition of the above two morphisms makes the pair (54) into an open-closed homotopy Lie 3algebra, i.e. defines (see 4.3 )

(i) a $L_{\infty}\{2\}$-structure, $\nu=\left\{\nu_{n}: \odot^{n}\left(X_{1}[3]\right) \rightarrow X_{2}[4]\right\}_{n \geq 1}$, on $X_{1}$, i.e. an ordinary $L_{\infty}$-structure on $X_{1}[2]$; as restriction of the propagator $\omega_{K}$ to the inner 2 -sphere in $\bar{C}_{2,0}\left(\mathbb{H}^{3}\right)$ is the standard homogeneous volume form on $S^{2}$, we conclude by Corollary 8.2.5.1 that this $L_{\infty}\{2\}$-structure is precisely the Poisson-Schouten bracket $\{,\}_{-2}$ in $X_{1}$;

(ii) a $L_{\infty}\{1\}$-structure, $\mu=\left\{\mu_{0, n}: \odot^{n}\left(X_{2}[2]\right) \rightarrow X_{1}[3]\right\}_{n \geq 1}$, on $X_{2}$; this structure is given by graphs $\Gamma$ whose vertices lie in $\mathbb{C}=\partial \overline{\mathbb{H}^{3}}$; as weights of all such graphs w.r.t. $\omega_{K}$ are equal to zero, we conclude that this $L_{\infty}\{1\}$-structure is trivial, i.e. all operations $\mu_{0, n}=0$ for $n \geq 2$;

(iii) a $L_{\infty}$-morphism, $F$, of Lie algebras,

$$
F:\left(X_{1},\{,\}_{-2}\right) \longrightarrow\left(\operatorname{Coder}\left(\odot^{\bullet}\left(X_{2}[2]\right)\right),[,]\right)
$$

given by sums over graphs $\Gamma \in \mathfrak{G}^{\downarrow}$. Note that as $\operatorname{deg} \omega_{K}=2$, only those graphs contribute to $F$ which satisfy the condition $3 n+2 m-3=2 l$, where $n$ is the number of vertices of $\Gamma$ lying in $\mathbb{H}^{3}, m$ is the number of vertices of $\Gamma$ lying in the boundary plane $\mathbb{C}=\partial \overline{\mathbb{H}^{3}}$ and $l$ is the number of edges of $\Gamma$; put another way, $l=3 k+m$ and $n=2 k+1$ for some $k \in \mathbb{N}$.

At the first glance it might seem that, contrary to the case of $\bar{C}(\mathbb{H})$ where the graph (53) encodes a natural graded commutative structure in $X_{2}=\mathcal{O}_{V}$, there is no graph with non-zero weight in the case of $\bar{C}\left(\mathbb{H}^{3}\right)$-theory which would encode the canonical $\operatorname{Lie}\{1\}$ structure on $X_{2}=\mathcal{T}_{\text {poly }}(V)$. However, this is not quite so: if $\gamma$ is an arbitrary MC element of the Poisson-Schouten 2-algebra $\left(X_{1}=\mathfrak{g}_{3}\left(V^{*}[1] \oplus V^{)},\{,\}_{-2}\right)\right.$, then the aforementioned $L_{\infty}$-morphism $F$ sends $\gamma$ into an MC element, $F(\gamma)$, of the Lie algebra $\left(\operatorname{Coder}\left(\odot \bullet\left(X_{2}[2]\right)\right)\right.$, [ , ]) which is the same as a $L_{\infty}\{1\}$-structure on $X_{2}=\mathcal{T}_{\text {poly }}(V)$. If we take $\gamma=\delta$, then the only graph contributing to $F(\delta)$ is the following one

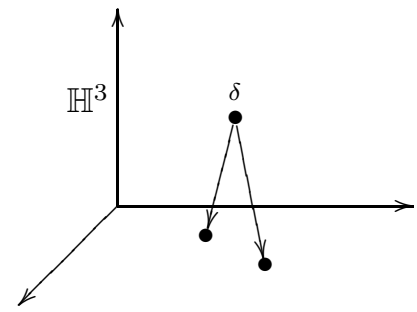

and has weight 1. Therefore, $F(\delta)$ is nothing but the ordinary Schouten structure, $\{\bullet\}_{-1}$, in $\mathcal{T}_{\text {poly }}(V)$ ! The conclusion is that any MC element, $\gamma$, in the $d g$ Lie algebra

$$
\left(\mathfrak{g}_{3}\left(V^{*}[1] \oplus V\right),\{,\}_{-2}, d=\{\delta,\}_{-2}\right)
$$

gives a deformation, $F(\gamma)$, of the Schouten bracket in $\mathcal{T}_{\text {poly }}(V)$. As the former dg Lie algebra is cohomologically trivial, any such a deformation of $\{\bullet\}_{-1}$ must be homotopy trivial. Still homotopy trivial does not mean trivial, and we can get some funny deformations of $\left(\mathcal{T}_{\text {poly }}(V),\{\bullet\}_{-1}\right)$ in this way.

9.5.1. Proposition. To any Lie coalgebra structure,

$$
\Delta: V \longrightarrow V \wedge V
$$

in a vector space $V=\mathbb{R}^{d}$ there corresponds an $M C$ element in the $d g$ Lie algebra

$$
\left(\mathfrak{g}_{3}\left(V^{*}[1] \oplus V\right) \simeq \mathbb{R}\left[\left[x^{\alpha}, \psi_{\alpha}, \eta^{\alpha}, y_{\alpha}\right]\right],\{,\}_{-2}, d=\{\delta,\}_{-2}\right),
$$


given explicitly by the following formal power series

$$
\gamma^{\Delta}:=\frac{1}{2} \sum_{\alpha, \beta, \text { delta }} C_{\alpha \beta}^{\delta} \eta^{\alpha} \eta^{\beta} y_{\delta}+\sum_{\alpha, \beta}\left(\sum_{n=1}^{\infty} \frac{B_{n}}{n !} \sum_{\xi_{i}, \zeta_{i}} C_{\alpha \xi_{1}}^{\zeta_{1}} C_{\zeta_{1} \xi_{2}}^{\zeta_{2}} \cdots C_{\zeta_{n-1} \xi_{n}}^{\beta} x^{\xi_{1}} x^{\xi_{2}} \cdots x^{\xi_{n}}\right) \eta^{\alpha} y_{\beta}
$$

where $C_{\alpha \beta}^{\delta} \in \mathbb{R}$ are the structure constants of $\Delta$ in a basis $x^{\alpha}$,

$$
\Delta\left(x^{\delta}\right)=\sum_{\alpha \beta} C_{\alpha \beta}^{\delta} x^{\alpha} x^{\beta}
$$

and $B_{n}$ are the Bernoulli numbers, $B_{1}=-\frac{1}{2}, B_{2}=\frac{1}{6}, B_{3}=0, B_{4}=-\frac{1}{30}$, etc.

Proof. Set

$$
\gamma:=-\frac{1}{2} \sum_{\alpha, \beta, \delta} C_{\alpha \beta}^{\delta} \eta^{\alpha} \eta^{\beta} \psi_{\delta}+\sum_{\alpha, \beta} C_{\alpha}^{\beta}(x) \eta^{\alpha} y_{\beta}
$$

for some $C_{\alpha}^{\beta}(x) \in \mathbb{R}\left[\left[x^{\alpha}\right]\right]$. This degree 3 element satisfies the MC equation in (55),

$$
d \gamma+\frac{1}{2}\{\gamma, \gamma\}_{-2}=0
$$

if and only if

$$
C_{\alpha \xi}^{\delta} C_{\beta \gamma}^{\xi}+C_{\gamma \xi}^{\delta} C_{\alpha \beta}^{\xi}+C_{\beta \xi}^{\delta} C_{\gamma \alpha}^{\xi}=0
$$

and the series $\hat{C}_{\alpha}^{\beta}(x):=\delta_{\alpha}^{\beta}+C_{\beta}^{\alpha}(x)$ satisfies the equations,

$$
C_{\alpha \beta}^{\gamma} \hat{C}_{\gamma}^{\delta}(x)=\hat{C}_{\alpha}^{\gamma}(x) \partial_{\gamma} \hat{C}_{\beta}^{\delta}(x)-\hat{C}_{\beta}^{\gamma}(x) \partial_{\gamma} \hat{C}_{\alpha}^{\delta}(x) .
$$

The first equations are the co-Jacobi identities for $\Delta$. The second equations have a nice geometric meaning. Let $\mathfrak{g}:=V^{*}$ be the Lie algebra dual to the coalgebra $V$; in a basis $\left\{e_{\alpha}\right\}$ of $\mathfrak{g}$ dual to $\left\{x^{\alpha}\right\}$ the Lie algebra structure is given by,

$$
\left[e_{\alpha}, e_{\beta}\right]=\sum_{\gamma} C_{\alpha \beta}^{\gamma} e_{\gamma} .
$$

Equations (56) are equivalent to saying that the map,

$$
\begin{array}{rlc}
f: \quad \mathfrak{g} & \longrightarrow & \mathcal{T}_{\mathfrak{g}} \\
e_{\alpha} & \longrightarrow \sum_{\beta} \hat{C}_{\alpha}^{\beta}(x) \frac{\partial}{\partial x^{\beta}}
\end{array}
$$

is a map of Lie algebras. Here $\mathcal{T}_{\mathfrak{g}}$ is the Lie algebra of formal vector fields on $\mathfrak{g}$, i.e. derivations of the completed symmetric tensor algebra $\widehat{\odot} \mathrm{V}$. It was proven in [Me1] (see Corollary 4.1.2 there) that for any Lie algebra $\mathfrak{g}$ such a canonical map exists and is given by the formulae

$$
\hat{C}_{\alpha}^{\beta}(x)=\delta_{\alpha}^{\beta}+\sum_{n=1}^{\infty} \frac{B_{n}}{n !} \sum_{\xi_{i}, \zeta_{i}} C_{\alpha \xi_{1}}^{\zeta_{1}} C_{\zeta_{1} \xi_{2}}^{\zeta_{2}} \cdots C_{\zeta_{n-1} \xi_{n}}^{\beta} x^{\xi_{1}} x^{\xi_{2}} \cdots x^{\xi_{n}}
$$

This fact completes the proof.

The Schouten brackets and the wedge product of polyvector fields make $\mathcal{T}_{\text {poly }}\left(\mathbb{R}^{d}\right) \simeq \mathbb{R}\left[\left[x^{\alpha}, \psi_{\alpha}\right]\right]$ into a Gerstenhaber algebra so that the Schouten bracket, $\{\bullet\}_{-1}$, is uniquely determined by its values on the generators $x^{\alpha}$ and $\psi_{\alpha}$,

$$
\begin{aligned}
& \left\{x^{\alpha} \bullet x^{\beta}\right\}_{-1}=0, \\
& \left\{\psi_{\alpha} \bullet x^{\beta}\right\}_{-1}=\delta_{\beta}^{\alpha}, \\
& \left\{\psi_{\alpha} \bullet \psi_{\beta}\right\}_{-1}=0 .
\end{aligned}
$$

9.5.1. Corollary. For any Lie coalgebra structure on $V=\mathbb{R}^{d}$ the following formulae,

$$
\begin{aligned}
\left\{x^{\alpha} \bullet x^{\beta}\right\}_{-1} & =0 \\
\left\{\psi_{\alpha} \bullet x^{\beta}\right\}_{-1} & =\delta_{\beta}^{\alpha}+\sum_{n=1}^{\infty} \frac{B_{n} \hbar^{n}}{n !} \sum_{\xi_{i}, \zeta_{i}} C_{\alpha \xi_{1}}^{\zeta_{1}} C_{\zeta_{1} \xi_{2}}^{\zeta_{2}} \cdots C_{\zeta_{n-1} \xi_{n}}^{\beta} x^{\xi_{1}} x^{\xi_{2}} \cdots x^{\xi_{n}} \\
\left\{\psi_{\alpha} \bullet \psi_{\beta}\right\}_{-1} & =\hbar \sum_{\gamma} C_{\alpha \beta}^{\gamma} \psi_{\gamma}
\end{aligned}
$$


give a 1-parameter deformation of the standard Gerstenhaber algebra structure on $\mathcal{T}_{\text {poly }}(V)$.

\section{Towards the theory of open-closed morphisms of deformation quantizations}

10.1. De Rham field theory on the first model of $\widehat{\mathfrak{C}}_{\bullet}(\mathbb{C})$. In $₫ 5$ we introduced two different configuration space models for the operad $\mathcal{M} \operatorname{or}\left(L_{\infty}\right), \widehat{\mathfrak{C}}(\mathbb{C})$ and $\widehat{C}(\mathbb{H})$, the first being a compactification of the quotient space $\operatorname{Conf}_{n}(\mathbb{C}) /\{z \rightarrow z+\mathbb{C}\}$ and the second being that of the space $\operatorname{Conf}_{n}(\mathbb{H}) /\left\{z \rightarrow \mathbb{R}^{+} z+\mathbb{R}\right\} ;$ moreover, each model was equipped with two non-isomorphic semialgebraic structures. In [Me2 we studied de Rham field theories on $\widehat{C}(\mathbb{H})$ and obtained some exotic $L_{\infty}$-automorphisms of the Lie algebra $\mathcal{T}_{\text {poly }}\left(\mathbb{R}^{d}\right)$ for any $d$. In this section we outline an analogous theory for the compactification $\widehat{\mathfrak{C}}(\mathbb{C})$.

Consider the 2-coloured operad of semialgebraic manifolds

$$
\widehat{\mathfrak{C}}(\mathbb{C}):=\bar{C} \cdot(\mathbb{C}) \sqcup \widehat{\mathfrak{C}}_{\bullet}(\mathbb{C}) \sqcup \bar{C} \cdot(\mathbb{C})
$$

equipped with a semialgebraic structure given by the embedding (19), and let

$$
\mathfrak{G}_{\widehat{\mathfrak{C}}(\mathbb{C})}=\mathfrak{G}_{\bar{C} \cdot(\mathbb{C})} \bigoplus \mathfrak{G}_{\widehat{\mathfrak{C}}_{\bullet}(\mathbb{C})} \bigoplus \mathfrak{G}_{\bar{C} \cdot(\mathbb{C})}
$$

be an associated 2-coloured operad operad of Feynman diagrams. Each summand in the latter direct sum is spanned by (equivalence classes of) directed graphs satisfying all the conditions given in 97.1 the only important difference from the definition of $\mathfrak{G}$ in $₫ \mathbf{7 . 1}$ is that graphs in each summand should be understood as drawn on the corresponding summand of the operad $\widehat{\mathfrak{C}}(\mathbb{C})$ and equipped thereby with the corresponding colour. We leave to the reader as an exercise to write down explicitly composition rules in $\mathfrak{G}_{\widehat{\mathfrak{C}}(\mathbb{C})}$ with the help of the basic 1-coloured formula (34).

A de Rham field theory on $\widehat{\mathfrak{C}}(\mathbb{C})$, that is a morphism of dg cooperads,

$$
\Omega:\left(\check{\mathfrak{G}}_{\widehat{\mathfrak{C}}(\mathbb{H})}, 0\right) \longrightarrow\left(\Omega_{\widehat{\mathfrak{C}}(\mathbb{C})}, d_{D R}\right),
$$

is the same as

(i) a pair of de Rham field theories on $\bar{C} \bullet(\mathbb{C})$, i.e. a pair of maps

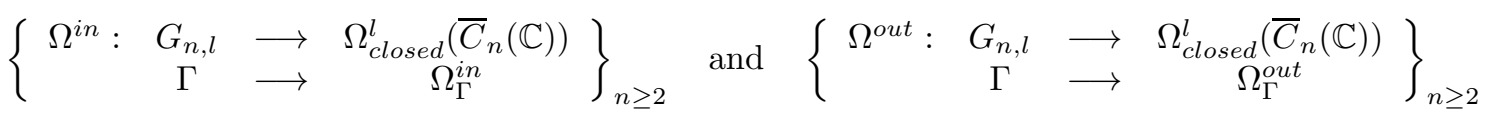

satisfying on the boundary strata of $\bar{C}_{n}(\mathbb{C})$ the factorization property (42), and

(ii) a map

$$
\left\{\begin{array}{ccc}
\Xi: G_{n, l} & \longrightarrow & \Omega_{\text {closed }}^{l}\left(\widehat{\mathfrak{C}}_{n}(\mathbb{C})\right) \\
\Gamma & \Xi_{\Gamma}
\end{array}\right\}_{n \geq 2}
$$

such that $\Xi_{\Gamma_{o p p}}=-\Xi_{\Gamma}$ and, for any $\Gamma \in \mathcal{G}_{n, 2 n-3}$, and any boundary embeddings

$j_{A}: \widehat{\mathfrak{C}}_{n-\# A+1}(\mathbb{C}) \times \bar{C}_{\# A}(\mathbb{C}) \hookrightarrow \widehat{\mathfrak{C}}_{n}(\mathbb{C}), \quad j_{A_{1}, \ldots, A_{k}}: \bar{C}_{k}(\mathbb{C}) \times \widehat{\mathfrak{C}}_{n-\# A_{1}+1}(\mathbb{C}) \times \ldots \times \widehat{\mathfrak{C}}_{n-\# A_{k}+1}(\mathbb{C}) \hookrightarrow \widehat{\mathfrak{C}}_{n}(\mathbb{C})$

one has

$$
\begin{aligned}
& j_{A}^{*}\left(\Xi_{\Gamma}\right) \simeq(-1)^{\sigma_{A}} \Xi_{\Gamma / \Gamma_{A}} \wedge \Omega_{\Gamma_{A}}^{i n}, \\
& j_{A_{1}, \ldots, A_{k}}^{*}\left(\Xi_{\Gamma}\right) \simeq(-1)^{\sigma_{A_{1}, \ldots, A_{k}}} \Omega_{\Gamma /\left\{\Gamma_{A_{1}}, \ldots, \Gamma_{A_{k}}\right\}}^{\text {out }} \wedge \Xi_{\Gamma_{A_{1}}} \wedge \ldots \wedge \Xi_{\Gamma_{A_{k}}},
\end{aligned}
$$

where the sign $(-1)^{\sigma_{A_{1}} \ldots A_{k}}$ is defined by the equality

$$
\mathcal{O}_{\Gamma}=(-1)^{\sigma_{A_{1} \ldots A_{k}}} \mathcal{O}_{\Gamma /\left\{\Gamma_{A_{1}}, \ldots, \Gamma_{A_{k}}\right\}} \wedge \mathcal{O}_{\Gamma_{A_{1}}} \wedge \ldots \wedge \mathcal{O}_{\Gamma_{A_{k}}},
$$

i.e. it is given just by a rearrangement of the wedge product of edges of $\Gamma$. 
The space $\widehat{\mathfrak{C}}_{2}(C)$ is the cylinder $S^{1} \times[0,+\infty]$, see (23), whose boundaries are circles $S_{\text {in }}^{1}$ and, respectively, $S_{\text {out }}^{1}$, corresponding to two points moving too close to each other and, respectively, to two points moving too far away from each. For any pair of integers $i, j \in[n], i \neq j$, there is an associated forgetting map,

$$
\begin{array}{ccc}
p_{i j}: & \mathfrak{C}_{n}(\mathbb{C}) & \longrightarrow \\
\left(z_{1}, \ldots, z_{n}\right) & \longrightarrow\left(z_{i}, z_{j}\right),
\end{array}
$$

which extends to a smooth map of their compactifications,

$$
p_{i j}: \widehat{\mathfrak{C}}_{n}(\mathbb{C}) \rightarrow \widehat{\mathfrak{C}}_{2}(\mathbb{C}) .
$$

Hence, for any closed differential 1 -form $\omega$ on $\widehat{C}_{2}$ the pull-back $p_{i j}^{*}(\omega)$ is a well-defined one-form on $\widehat{\mathfrak{C}}_{n}(\mathbb{C})$. In particular, for any graph $\Gamma \in G_{n, l}$ and any edge $e \in E(\Gamma)$ there is an associated differential form $p_{e}^{*}(\omega) \in \Omega^{1}\left(\widehat{\mathfrak{C}}_{n}(\mathbb{C})\right)$, where $p_{e}:=p_{i j}$ if the edge $e$ begins at the vertex labelled by $i$ and ends at the vertex labelled by $j$. Similar forgetful maps $\pi_{i j}$ and $\pi_{e}$ can be defined for the configuration spaces $\bar{C} \bullet(\mathbb{C})$.

Let $\omega$ be an arbitrary closed differential 1-form on $\widehat{\mathfrak{C}}_{2}(\mathbb{C})$ such that the restrictions

$$
\omega_{\text {in }}:=\left.\omega\right|_{S_{\text {in }}^{1}} \text { and } \quad \omega_{\text {out }}:=\left.\omega\right|_{S_{\text {out }}^{1}}
$$

define cohomologically non-trivial 1-forms on the circle normalized so that

$$
\int_{S^{1}} \omega_{\text {in }}=\int_{S^{1}} \omega_{\text {out }}=2 \pi
$$

We call such a differential form a propagator on $\widehat{\mathfrak{C}}_{2}(\mathbb{C})$. Define a series of maps,

$$
\begin{aligned}
& \Omega^{\text {in }}: G_{n, l} \rightarrow \quad \Omega^{l}\left(\widehat{\mathfrak{C}}_{n}(\mathbb{C})\right) \quad \Omega^{\text {out }}: G_{n, l} \rightarrow \quad \Omega^{l}\left(\widehat{\mathfrak{C}}_{n}(\mathbb{C})\right) \\
& \Gamma \rightarrow \Omega_{\Gamma}^{i n}:=\bigwedge_{e \in E(\Gamma)} \frac{\pi_{e}^{*}\left(\omega_{\text {in }}\right)}{2 \pi} \quad \Gamma \rightarrow \Omega_{\Gamma}^{i n}:=\bigwedge_{e \in E(\Gamma)} \frac{\pi_{e}^{*}\left(\omega_{\text {out }}\right)}{2 \pi}
\end{aligned}
$$

and

$$
\begin{aligned}
\Xi: G_{n, l} & \longrightarrow \quad \Omega^{l}\left(\widehat{C}_{n, 0}\right) \\
\Gamma \quad & \longrightarrow \Xi_{\Gamma}:=\bigwedge_{e \in E(\Gamma)} \frac{p_{e}^{*}(\omega)}{2 \pi} .
\end{aligned}
$$

10.1.1. Theorem. For any propagator on $\widehat{\mathfrak{C}}_{2}(\mathbb{C})$ the associated data (61)-(62) define a de Rham field theory on the semialgebraic operad $\widehat{\mathfrak{C}}(\mathbb{C})$.

Proof. Equation (57) is equivalent to the following one,

$$
\int_{\widehat{\mathfrak{C}}_{(n-\# A) \sqcup \bullet}(\mathbb{C}) \times \bar{C}_{\# A}(\mathbb{C})} j_{A}^{*}\left(\Xi_{\Gamma}\right)=(-1)^{\sigma_{A}} \int_{\widehat{\mathfrak{C}}_{(n-\# A) \sqcup \bullet}(\mathbb{C})} \Xi_{\Gamma / \Gamma_{A}} \int_{\bar{C}_{\# A}(\mathbb{C})} \Omega_{\Gamma_{A}}^{i n} .
$$

Studying the embedding $\widehat{\mathfrak{C}}_{(n-\# A) \sqcup \bullet}(\mathbb{C}) \times \bar{C}_{\# A}(\mathbb{C}) \hookrightarrow \widehat{\mathfrak{C}}_{n}(\mathbb{C})$ in local coordinates defined by metric graphs, one easily sees that both sides of the above equation are zero unless $\Gamma_{A}$ has $2 \# A-3$ edges (so that $\Omega_{\Gamma_{A}}^{i n}$ is a top degree form on $\bar{C}_{\# A}(\mathbb{C})$ ) in which case the equality is almost obvious (cf. the proof of Proposition 8.2.5.1).

Consider next, for a partition $[n]=A_{1} \sqcup \ldots \sqcup A_{k}$, the associated boundary stratum of the form,

$$
j_{A_{1}, \ldots, A_{k}}: C_{k}(\mathbb{C}) \times \mathfrak{C}_{A_{1}}(\mathbb{C}) \times \ldots \times \mathfrak{C}_{A_{k}}(\mathbb{C}) \hookrightarrow \widehat{\mathfrak{C}}_{n}(\mathbb{C}) .
$$

By definition, this is a subset of $\widehat{\mathfrak{C}}_{n}(\mathbb{C})$ obtained in the limit $\tau \rightarrow \infty$ from a class of configurations in $C o n f_{n}(\mathbb{C})$ determined by the data:

(a) a configuration $\tau \cdot p=\left(\tau z_{1}, \ldots, \tau z_{k}\right) \in \operatorname{Conf}_{n}(\mathbb{C})$ obtained from a standard configuration $p=\left(z_{1}, \ldots, z_{k}\right) \in$ $C_{n}^{s t}(\mathbb{C}) \simeq C_{k}(\mathbb{C})$ by $\tau$-dilation,

(b) a collection of configurations, $p_{1} \in \mathfrak{C}_{\# A_{1}}^{s t}(\mathbb{C}), \ldots, p_{k} \in \mathfrak{C}_{\# A_{k}}^{s t}(\mathbb{C}), 1 \leq i \leq k$, placed, respectively, at the positions $\tau z_{1}, \ldots, \tau z_{k}$ in $\mathbb{H}$, that is, a configuration $\left(\tau z_{1}+p_{1}, \ldots, \tau z_{k}+p_{k}\right) \in \operatorname{Conf}_{n}(\mathbb{C})$. 
We have, therefore,

$$
j_{A_{1}, \ldots, A_{k}}^{*}\left(\Xi_{\Gamma}\right)=(-1)^{\sigma_{A,, \ldots, A_{k}}} \bigwedge_{e \in E\left(\Gamma /\left\{\Gamma_{\left.\left.A_{1} \ldots \Gamma_{A_{k}}\right\}\right)}\right.\right.} \frac{\lim _{\tau \rightarrow+\infty} p_{e}^{*}(\omega)}{2 \pi} \prod_{i=1}^{k} \bigwedge_{e \in E\left(\Gamma_{A_{i}}\right)} \frac{\lim _{\tau \rightarrow+\infty} p_{e}^{*}(\omega)}{2 \pi} .
$$

As $p_{e}^{*}(\omega)$ is invariant under translations we have for each $i \in[k]$

$$
\lim _{\tau \rightarrow+\infty} \bigwedge_{e \in E\left(\Gamma_{A_{i}}\right)} \frac{p_{e}^{*}(\omega)}{2 \pi}=\bigwedge_{e \in E\left(\Gamma_{A_{i}}\right)} \frac{p_{e}^{*}(\omega)}{2 \pi}
$$

On the other hand, for any $e \in E\left(\Gamma /\left\{\Gamma_{A_{1}} \ldots \Gamma_{A_{k}}\right\}\right)$,

$$
\lim _{\tau \rightarrow+\infty} p_{e}^{*}(\omega)=\pi_{e}^{*}\left(\omega_{\text {out }}\right)
$$

so that

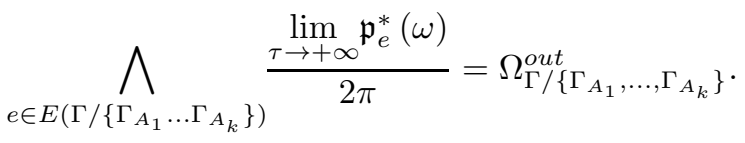

These two facts prove equality (58) and hence complete the proof of the Theorem.

10.1.2. Corollary. For any propagator $\omega$ on $\widehat{\mathfrak{C}}_{2}(\mathbb{C})$ and any graded vector space $V$ there are associated

(i) two $\mathcal{L}_{\infty}$-structures on $\mathcal{T}_{\text {poly }}(V)$,

$$
\begin{aligned}
\mu^{\text {in }}= & \left\{\mu_{n}^{\text {in }}: \odot^{n} \mathcal{T}_{\text {poly }}(V) \rightarrow \mathcal{T}_{\text {poly }}(V)[3-2 n]\right\}_{n \geq 2} \text { and } \mu^{\text {out }}=\left\{\mu_{n}^{\text {out }}: \odot^{n} \mathcal{T}_{\text {poly }}\left(\mathbb{R}^{d}\right) \rightarrow \mathcal{T}_{\text {poly }}\left(\mathbb{R}^{d}\right)[3-2 n]\right\}_{n \geq 2}, \\
& \text { given by formulae } 4 \text { 43)-444 for } \Omega=\Omega^{\text {in }} \text { and, respectively, } \Omega=\Omega^{\text {out }} ; \mu_{2}^{\text {in }} \text { and } \mu_{2}^{\text {out }} \text { coincide with the } \\
& \text { Schouten bracket; }
\end{aligned}
$$

(ii) a $\mathcal{L}_{\infty}$ morphism,

$$
F=\left\{\begin{array}{cccc}
F_{n}^{\text {Leib }}: & \odot^{n} \mathcal{T}_{\text {poly }}(V) & \longrightarrow & \mathcal{T}_{V}\left(\mathbb{R}^{d}\right)[2-2 n] \\
\gamma_{1} \otimes \ldots \otimes \gamma_{n} & \longrightarrow & F_{n}\left(\gamma_{1}, \ldots, \gamma_{n}\right)
\end{array}\right\}_{n \geq 1},
$$

from $\mu^{\text {in }}$-structure to $\mu^{\text {out }}$-structure given by the formulae,

$$
F_{n}\left(\gamma_{1}, \ldots, \gamma_{n}\right):=\left\{\begin{array}{cc}
\operatorname{Id} & \text { for } n=1 \\
\sum_{\Gamma \in \mathfrak{G}_{n, 2 n-2}} C_{\Gamma} \Phi_{\Gamma}\left(\gamma_{1}, \ldots, \gamma_{n}\right) & \text { for } n \geq 2
\end{array}\right.
$$

with

$$
C_{\Gamma}:=\int_{\widehat{\mathfrak{C}}_{n}(\mathbb{C})} \bigwedge_{e \in E(\Gamma)} \frac{p_{e}^{*}(\omega)}{2 \pi} .
$$

10.1.3. Remark. If $\omega$ is a propagator on $\widehat{\mathfrak{C}}_{2}(\mathbb{C})$ such that its restrictions (60) to both boundary circles coincide with the standard homogeneous volume form $\operatorname{dArg}\left(z_{1}-z_{2}\right)$ on $S^{1}$, then formulae (63) and (64) define a universal $\mathcal{L}_{\infty}$ automorphism of the Schouten algebra of polyvector fields. The propagator

$$
\omega\left(z_{1}, z_{2}\right)=\operatorname{dArg}\left(z_{1}-z_{2}\right)
$$

is well-defined on $\widehat{\mathfrak{C}}_{2}(\mathbb{C})$ and satisfies the aforementioned boundary conditions. However, all the weights (64) with $n \geq 2$ vanish in this case so that the associated automorphism $F$ is just the identity map. Any other smooth propagator on $\widehat{\mathfrak{C}}_{2}(\mathbb{C})$ is of the form

$$
\omega\left(z_{1}, z_{2}\right)=d \operatorname{Arg}\left(z_{1}-z_{2}\right)+d f\left(z_{1}, z_{2}\right)
$$

for some smooth (or semialgebraic) function $f$ on $\widehat{\mathfrak{C}}_{2}(\mathbb{C})$. It was proven in $\overline{\mathrm{Me} 2}$ that any such propagator defines a $\mathcal{L}_{\infty}$-automorphism of $\mathcal{T}_{\text {poly }}(V)$ which is homotopy equivalent to the trivial one. Thus the class of smooth propagators on $\widehat{\mathfrak{C}}_{2}(\mathbb{C})$ can not give us an exotic (i.e. homotopy non-trivial) universal automorphism of the Schouten algebra. Hence one should try using singular propagators for that purpose (cf. [Ko3]) which have at most simple polar singularity at the collapsing stratum $S^{1} \subset \widehat{\mathfrak{C}}_{2}(\mathbb{C})$. We conjecture that

$$
\omega\left(z_{1}, z_{2}\right):=\frac{1}{\mathfrak{i}} d \log \frac{z_{1}-z_{2}}{1+\left|z_{1}-z_{2}\right|}
$$


is an example of such suitable propagator on $\widehat{\mathfrak{C}}_{2}(\mathbb{C})$ which gives us an exotic universal $L_{\infty}$-automorphism of the Schouten algebra via Corollary 10.1.2. We hope to discuss elsewhere our motivation for that conjecture, and its relation to the Deligne-Drinfeld conjecture on the structure of the Grothendieck-Teichmüller algebra grt.

De Rham field theories on the upper half space model of $\mathcal{M o r}\left(L_{\infty}\right)$ have been studied in Me2].

10.2. On (auto)morphisms of deformation quantizations. Any de Rham field theory, $\Omega$, on the 4-coloured operad,

$$
\widehat{\mathfrak{C}}(\mathbb{H}):=\underbrace{\bar{C}_{\bullet}(\mathbb{C}) \bigsqcup \bar{C}_{\bullet}, \bullet(\mathbb{H})}_{\text {in }} \bigsqcup \widehat{\mathfrak{C}}_{\bullet, \bullet}(\mathbb{H}) \bigsqcup \widehat{\mathfrak{C}}_{\bullet}(\mathbb{C}) \bigsqcup \underbrace{\bar{C}_{\bullet}(\mathbb{C}) \bigsqcup \bar{C}_{\bullet},(\mathbb{H})}_{\text {out }},
$$

gives us an open-closed homotopy morphism between the two deformation quantizations corresponding to the "in" and "out" colours, respectively.

It follows from Tamarkin's proof of Kontsevich formality theorem that the Grothendieck-Teichmüller group, GRT, acts (up to homotopy) on deformation quantizations. Following the general philosophy one can expect that this action can be explicitly presented as an open-closed homotopy morphism determined by a propagator $\omega \in \Omega_{\widehat{\mathfrak{C}}_{2,0}(\mathbb{H})}^{1}$ which vanishes on all boundary strata of $\widehat{\mathfrak{C}}_{2,0}(\mathbb{H})$ except the inner cylinders, both Kontsevich eyes and the spaces $B_{1}$ and $B_{2}$ shown in the following picture

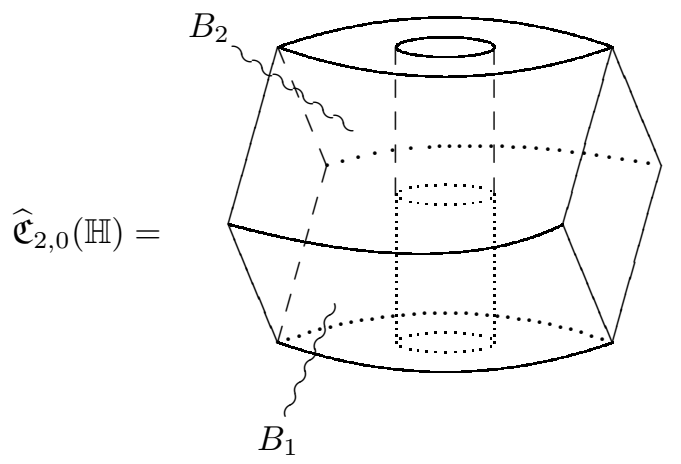

It is not hard to construct such a de Rham field theory on $\widehat{\mathfrak{C}}(\mathbb{H})$ out of a smooth propagator on $\widehat{\mathfrak{C}}_{2,0}(\mathbb{H})$ along the lines explained in the previous section; however, as $\widehat{\mathfrak{C}}_{2,0}(\mathbb{H})$ is contractible to the topological circle, any such a theory gives us a homotopy trivial open-closed transformation. Therefore again only singular propagators can, in principle, give us explicit formulae for the action of GRT on deformation quantizations. One such singular propagator on $\bar{C}$ •,• $(\mathbb{H})$ was introduced by Kontsevich in $[\mathrm{Ko}]$ but a rigorous proof of his claim that this propagator works indeed is not yet available in the literature (to the best knowledge of the author).

\section{Appendix A. Operads and coloured operads [BM, GJ, GK, LV]}

A.1. Trees. Let $\mathcal{T}$ be the set of all possible connected genus 0 graphs constructed from the following 1-vertex directed graphs called $n$-corollas,

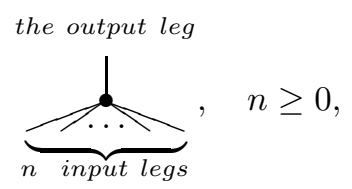

by taking their disjoint unions then and gluing some output legs to the same number of input legs. The resulting graph is called a tree. The glued legs are called the edges of the tree, and all the rest legs are called the legs of the tree. Each tree $T$ has, by construction, a unique output leg. The set of edges of $T$ is denoted by $E(T)$, the set of vertices by $V(T)$, and the set of input legs by $L(T)$. If $\# L(T)=n$, then $T$ is called an $n$-tree. The subset of $\mathcal{T}$ consisting of $n$-trees is denoted by $\mathcal{T}_{n}$. Note that every edge as well as every leg of a tree is naturally directed; we tacitly assume in all our pictures that the direction flow runs from the bottom to the top. For a vertex $v$, we denote by $I n_{v}$ the set of its input legs.

Let $I$ be a finite set. An $I$-tree is an \#I-tree equipped with a bijection $I \rightarrow L(T)$. The set of $I$-trees is denoted by $\mathcal{T}_{I}$. 
A.2. $\mathcal{S}$-modules. Let $\mathcal{C}$ be a symmetric monoidal category with the tensor product denoted by $\otimes$ and the unit object denoted by $\mathbb{1}$. We assume that the category $\mathcal{C}$ has small limits and colimits, and that for any object $O$ the functor $O \otimes$ preserves colimits.

Let $\mathcal{S}$ be the groupoid of finite sets. A functor $\mathcal{O}: \mathcal{S} \rightarrow \mathcal{C}$ is called an $\mathcal{S}$-module. The subcategory, $\mathbb{S}$, of $\mathcal{S}$ whose objects are the sets $[n], n \in \mathbb{N}$, and morphisms are the permutation groups $\mathbb{S}_{n}$ is the full sceleton of $\mathcal{S}$. The restriction of $\mathcal{O}$ to $\mathbb{S}$ is called an $\mathbb{S}$-module. One can reconstruct $\mathcal{O}$ from its restriction to $\mathbb{S}$ by setting $\mathcal{O}(I)$ to be the colimit

$$
\mathcal{O}(I):=\left(\bigoplus_{[\# I] \rightarrow I} \mathcal{O}([\# I])\right)_{\mathbb{S}_{\# I}} .
$$

Given an $\mathcal{S}$-module $\mathcal{O}$ and a tree $T$, one constructs a decorated tree $T\langle\mathcal{O}\rangle$ as the colimit,

$$
T\langle\mathcal{O}\rangle:=\left(\bigoplus_{\sigma:[\# V(T)] \rightarrow V(T)} \mathcal{O}\left(\operatorname{In}_{\sigma(1)}\right) \otimes \mathcal{O}\left(\operatorname{In}_{\sigma(2)}\right) \otimes \ldots \otimes \mathcal{O}\left(\operatorname{In}_{\sigma(\# V(T))}\right)\right)_{\mathbb{S}_{\# V(T)}},
$$

and then defines an $\mathcal{S}$-module, $\mathcal{T}\langle\mathcal{O}\rangle: \mathcal{S} \rightarrow \mathcal{C}$, which is given on a finite set $I$ as the following colimit,

$$
\mathcal{T}\langle\mathcal{O}\rangle(I):=\bigoplus_{T \in \mathcal{T}_{I}} T\langle\mathcal{O}\rangle
$$

The association $\mathcal{T}: \mathcal{O} \rightsquigarrow \mathcal{T}\langle\mathcal{O}\rangle$ is an endofunctor in the category of $\mathcal{S}$-modules which comes canonically equipped with a natural transformation $t: \mathcal{T} \circ \mathcal{T} \rightarrow \mathcal{T}$ as, for any finite set $I$, there is a natural in $\mathcal{O}$ "tautological" map

$$
\mathcal{T}\langle\mathcal{T}\langle\mathcal{O}\rangle\rangle(I) \longrightarrow \bigoplus_{T \in \mathcal{T}_{I}} T\langle\mathcal{O}\rangle=\mathcal{T}\langle\mathcal{O}\rangle
$$

which sends a tree $T^{\prime} \in \mathcal{T}\langle\mathcal{T}\langle\mathcal{O}\rangle\rangle$ whose vertices, $v$, are decorated by some $\mathcal{O}$-decorated trees, $T_{v}^{\prime \prime} \in \mathcal{T}\langle\mathcal{O}\rangle$, into the $\mathcal{O}$-decorated tree $T$ obtained from $T^{\prime}$ by replacing each $v$ with $T_{v}^{\prime \prime}$.

\section{A.3. Definitions of an operad.}

A.3.1. First definition. A non-unital operad is an $\mathcal{S}$-module, $\mathcal{O}$, together with a natural transformation,

$$
\mu: \mathcal{T}\langle\mathcal{O}\rangle \longrightarrow \mathcal{O}
$$

such that the diagrams,

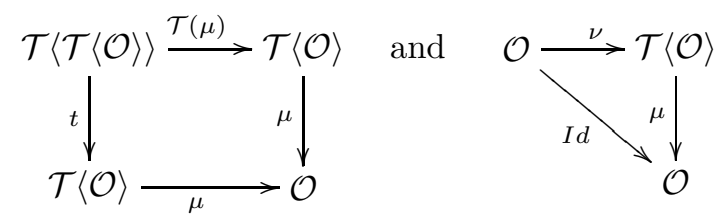

commute. Here $\nu: \mathcal{O} \rightarrow\langle\mathcal{T}\rangle$ stands for the trasnformation which identifies $\mathcal{O}(I)$ with the decorated $I$-corolla. We omit the definition of a morphism of (non-unital) operads as it is obvious.

A.3.2. Example. For any $\mathcal{S}$-module $\mathcal{E}$ the $\mathcal{S}$ module $\mathcal{T}\langle\mathcal{E}\rangle$ has a natural structure of a non-unital operad called the free operad generated by $\mathcal{E}$.

A.3.3. Example. For any vector space $V$ the $\mathbb{S}$-module $\mathcal{E} n d_{V}:=\left\{\operatorname{Hom}\left(V^{\otimes n}, V\right)\right\}_{n \geq 0}$ has a natural structure of an operad called the endomorphism operad of $V$.

Let $\uparrow$ denote the exceptional tree without vertices, and set

$$
\mathcal{T}^{+}:=\mathcal{T} \sqcup \uparrow
$$

be the enlarged family of trees. For any $\mathcal{S}$-module $\mathcal{O}$ set the decorated graph $\uparrow\langle\mathcal{O}\rangle$ to be $\mathbb{1}$, the unit in the category $\mathcal{C}$.

An operad with unit is defined by replacing in the as bove definition of a non-unital operad the symbol $\mathcal{T}$ by the symbol $\mathcal{T}^{+}$. 
A.3.4. Second definition. A non-unital operad is an $\mathcal{S}$-module, $\mathcal{O}$, together with a family of natural transformations,

$$
\left\{\mu_{T}: T\langle\mathcal{O}\rangle \longrightarrow \mathcal{O}\right\}_{T \in \mathcal{T}}
$$

parameterized by all possible tress from the family $\mathcal{T}$ such that

$$
\mu_{T}=\mu_{T / T^{\prime}} \circ \tilde{\mu}_{T^{\prime}}
$$

for any subtree $T^{\prime} \subset T$. Here $T / T^{\prime}$ stands for the tree obtained from $T$ by shrinking the whole subtree $T^{\prime}$ into a single $L\left(T^{\prime}\right)$-corolla, and $\tilde{\mu}_{T^{\prime}}: T\langle\mathcal{O}\rangle \rightarrow\left(T / T^{\prime}\right)\langle\mathcal{O}\rangle$ stands for the natural transformation which equals $\mu_{T^{\prime}}$ on the decorated vertices lying in $T$ and which is identity on all other vertices of $T$. Enlarging the family of trees from $\mathcal{T}$ to $\mathcal{T}^{+}$as above, one obtains similarly a definition of an operad with unit.

A.3.5. Third definition. A non-unital operad is an $\mathcal{S}$-module, $\mathcal{O}$, together with a family of natural transformations,

$$
\left\{\mu_{T}: T\langle\mathcal{O}\rangle \longrightarrow \mathcal{O}\right\}_{T \in \mathcal{T}^{\text {red }}}
$$

parameterized by the subfamily $\mathcal{T}^{\text {red }} \subset \mathcal{T}$ of 2 -vertex trees such that for any three vertex tree $\bar{T}$ the diagram

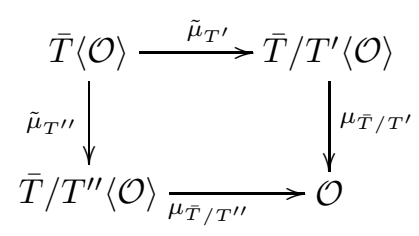

commutes. Here $T^{\prime}$ and $T^{\prime \prime}$ stand for the two only possible 2-vertex subtrees of $\bar{T}$.

For arbitrary finite sets $I$ and $J$ let $T_{I}$ be the $I$-corolla, $T_{J}$ be the $J$-corolla, and, for any $i \in I$, let $T_{(I-i) \sqcup J}$ be the 2 -vertex tree obtained by gluing the output vertex of $T_{J}$ into the $i$-labeled input leg of $T_{I}$. The associated composition

is often denoted in the literature by $o_{i}^{I, J}$.

$$
\mu_{T_{(I-i) \sqcup J}}: \mathcal{O}(I) \otimes \mathcal{O}(J) \longrightarrow \mathcal{O}((I-i) \sqcup J)
$$

An operad with unit is, by definition, a non-unital operad equipped with a morphism $\mathbb{1} \bullet: \mathbb{1} \rightarrow \mathcal{O}(\bullet)$ for any one point set • such that the compositions

$$
\mathcal{O}(I) \rightarrow \mathcal{O}(I) \otimes \mathbb{1} \stackrel{\operatorname{Id} \otimes \mathbb{I} \bullet}{\longrightarrow} \mathcal{O}(I) \otimes \mathcal{O}(\bullet) \stackrel{\circ_{i}^{I} \bullet}{\longrightarrow} \mathcal{O}(I)
$$

and

$$
\mathcal{O}(I) \rightarrow \mathbb{1} \otimes \mathcal{O}(I) \stackrel{\mathbb{1} \bullet \otimes \operatorname{Id}}{\longrightarrow} \mathcal{O}(\bullet) \otimes \mathcal{O}(I) \stackrel{\bullet}{\longrightarrow} \mathcal{O} \mathcal{O}(I)
$$

are the identities for any finite set $I$ and any $i \in I$.

A.3.6. Fourth definition. A non-unital operad is an $\mathcal{S}$-module, $\mathcal{O}$, together with a family of natural transformations,

$$
\left\{\circ_{f}: \mathcal{O}(I) \otimes \bigotimes_{i \in I} \mathcal{O}\left(f^{-1}(i)\right) \longrightarrow \mathcal{O}(J)\right\}_{f: J \rightarrow I}
$$

parameterized by a family, $\{f: J \rightarrow I\}$, of surjections of finite sets, such that, for any triple $K \stackrel{g}{\rightarrow} J \stackrel{f}{\rightarrow} I$ the diagram

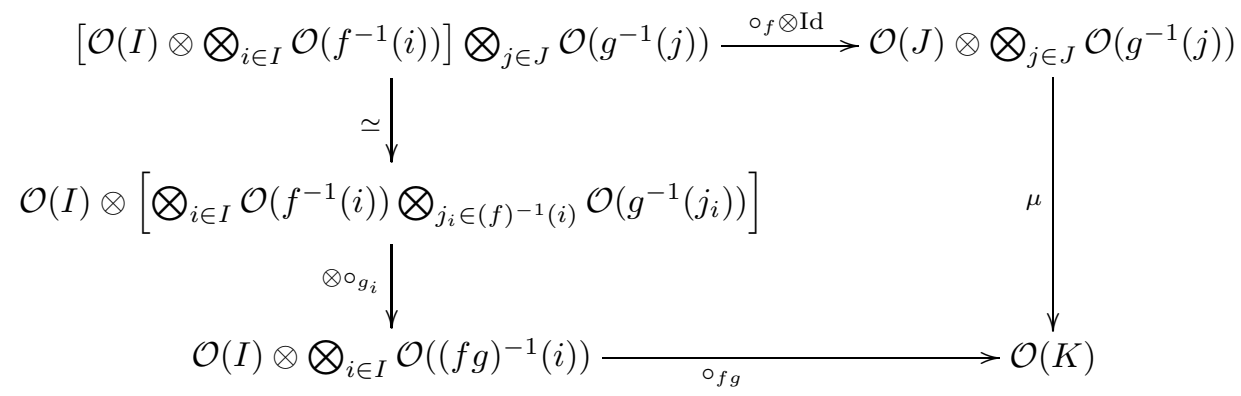


commutes. An operad with unit is, by definition, a non-unital operad equipped with a morphism $\mathbb{1} \bullet: \mathbb{1} \rightarrow \mathcal{O}(\bullet)$ for any one point set $\bullet$ such that the compositions

$$
\mathcal{O}(I) \rightarrow \mathcal{O}(I) \otimes \mathbb{1}^{\otimes \# I} \stackrel{\operatorname{Id} \otimes \mathbb{1}_{\rightarrow}^{\otimes \# I}}{\longrightarrow} \mathcal{O}(I) \otimes \mathcal{O}(\bullet)^{\otimes \# I} \stackrel{\circ_{I d}}{\longrightarrow} \mathcal{O}(I)
$$

and

$$
\mathcal{O}(I) \rightarrow \mathbb{1} \otimes \mathcal{O}(I) \stackrel{I_{\amalg}}{\longrightarrow} \mathcal{O}(I)
$$

are identities.

A.3.7. (Non)equivalences of definitions. All the four definitions of operads with unit are equivalent to each other. The first three definitions of non-unital operads are equivalent to each other, but not to the fourth definition. Every non-unital operad in the sense of the first three definitions is a non-unital operad in the sense of the fourth definition, but, obviously, not vice versa. A free non-unital operad in the sense of the fourth definition uses leveled trees rather than the ordinary ones.

In this paper we always understand a non-unital operad in the sense of any of the first three definitions. However, when we work with non-unital coloured operads we can in principle have a mixture of both approaches, one approach for one set of colours and another inequivalent approach for another set of colours. Such a mixture of two non-equivalent approaches does indeed happen in the geometric models for various operads of homotopy morphisms between homotopy algebras. We give a rigorous definition of that mixture below under the name of a non-unital coloured operad of transformation type.

A.4. Coloured operads. Let $\Phi$ be a set which we refer to as the set of colours. An n-corolla,

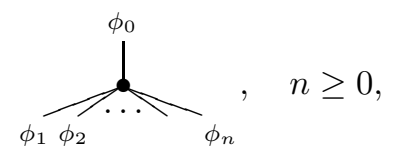

whose all legs are decorated with some (not-necessarily distinct) elements $\phi_{0}, \phi_{1}, \ldots, \phi_{n} \in \Phi$ is a called an $\Phi$ coloured $n$-corolla. If the set $\Phi$ consists just of a few elements, then we often make legs dashed or wiggy to indicate their colours, for example

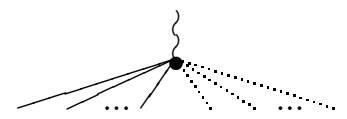

Let $\mathcal{T}^{\Phi}$ be the set of all possible connected genus 0 graphs constructed from $\Phi$-coloured corollas by taking their disjoint unions and then gluing some output legs with input legs of the same colour. The resulting graph is called a $\Phi$-coloured tree.

Now repeating all the first three definitions above with the symbol $\mathcal{T}$ replaced by $\mathcal{T}^{\Phi}$ we obtain three equivalent definitions of a (non-unital) $\Phi$-coloured operad in a symmetric monoidal category $\mathcal{C}$.

A.5. Coloured operads of transformation type. Many important examples of coloured operads come from ordinary operads and their modules.

Let $\mathcal{O}_{i n}$ and $\mathcal{O}_{\text {out }}$ be ordinary non-unital operads. An $\mathcal{S}$-module $\mathcal{M}$ is said to be a bimodule of transformation type over operads $\mathcal{O}_{\text {in }}$ and $\mathcal{O}_{\text {out }}$ if

(i) $\mathcal{M}$ is a right module over $\mathcal{O}_{\text {out }}$ in the sense of the first definitions of a non-unital operad, i.e. for any finite sets $I$ and $J$ and any $i \in I$ there is a morphism

$$
\circ_{i}^{I, J}: \mathcal{M}(I) \otimes \mathcal{O}_{i n}(J) \longrightarrow \mathcal{M}((I-i) \sqcup J)
$$

which is natural in $i, I$ and $J$ and satisfies obvious associativity conditions;

(ii) $\mathcal{M}$ is a right pseudo-module over $\mathcal{O}_{i n}$ in the sense of the fourth definition of a non-unital operad, i.e. for any surjection $f: J \rightarrow I$ there is a morphism,

$$
\circ_{f}: \mathcal{O}(I) \otimes \bigotimes_{i \in I} \mathcal{M}\left(f^{-1}(i)\right) \longrightarrow \mathcal{M}(J)
$$


such that, for any triple $K \stackrel{g}{\rightarrow} J \stackrel{f}{\rightarrow} I$ the diagram

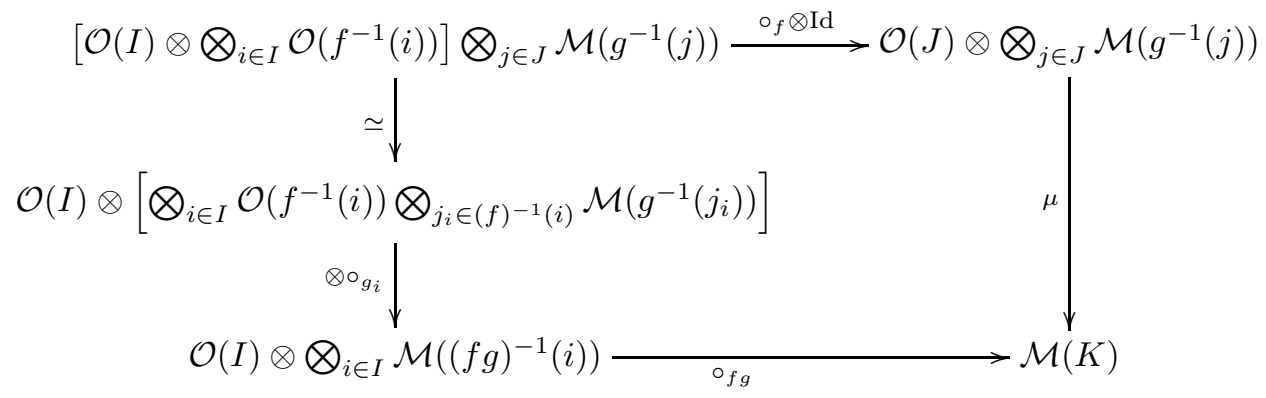

commutes.

The colimit $\mathcal{O}_{\text {in }} \oplus \mathcal{M} \oplus \mathcal{O}_{\text {out }}$ has then a natural structure of a non-unital two-coloured operad of mixed type which we call a non-unital coloured operad of transformation type. Such operads often occur when, for example, one is interested in universal morphisms from $\mathcal{O}_{\text {in }}$-algebras to $\mathcal{O}_{\text {out }}$-algebras. Propositions $\mathbf{3 . 1 . 1}$ and $\mathbf{5 . 1 . 1}$ describe typical examples of such 2-coloured operads.

The above notion can be straightforwardly generalized to the case when $\mathcal{O}_{\text {in }}$ and $\mathcal{O}_{\text {out }}$ are themselves non-unital coloured operads. The associated colimits $\mathcal{O}_{\text {in }} \oplus \mathcal{M} \oplus \mathcal{O}_{\text {out }}$ are also called non-unital coloured operad of transformation type. Theorem $\mathbf{6 . 2}$ describes an example.

Acknowledgement. It is a pleasure to thank Johan Alm, Johan Granåker and Carlo Rossi for valuable discussions. I am very grateful to the referee for a spotting a mistake in the original definition of the operad $\mathcal{G}^{\uparrow \downarrow}$ in $\S 7.1 .1$ and for numerous very useful suggestions and comments. 


\section{REFERENCES}

[A] J. Alm, Two-colored noncommmutative gerstenhaber formality and infinity Duflo isomorphism, preprint arXiv:1104.2194

[AT] A. Alekseev and C. Torossian, a preprint.

[BM] C. Berger and I. Moerdijk, Resolution of coloured operads and rectification of homotopy algebras, Contemp. Math. 431 (2007) $31-58$.

[CFFR] D. Calaque, G. Felder, A. Ferrario, and C. A. Rossi, Bimodules and branes in deformation quantization (2009), preprint arXiv:0908.2299.

[CaRo] D. Calaque and C. Rossi, Lectures on Duflo isomorphisms in Lie algebras and complex geometry, http://www.math.ethz.ch/u/felder/Teaching/AutumnSemester2007/Calaque

[Du] M. Duflo, Opèrateurs differentiels bi-invariants sur un groupe de Lie, Ann. Sci. ENS 10 (1977), 265-288.

[Ga] G. Gaiff, Compactifications of configuration spaces, In: Algebraic Geometry Seminars, 19981999 (Pisa), Scuola Normale Superiore di Pisa, Pisa, 1999, pp. 87-109.

[GJ] E. Getzler and J.D.S. Jones, Operads, homotopy algebra, and iterated integrals for double loop spaces, preprint hep-th/9403055

[GK] V. Ginzburg and M. Kapranov, Koszul duality for operads, Duke Math. J. 76 (1994) 203-272.

[HLTV] R. M. Hardt, P. Lambrechts, V. Turchin, and I. Volić, Real homotopy theory of semi-algebraic sets, preprint arXiv:0806.0476 (2008).

[Ho] E. Hoefel, On the coalgebra description of OCHA, preprint arXiv:math/0607435

[KaSt] T. Kajiura and J. Stasheff, Homotopy algebras inspired by classical open-closed string field theory, Commun. Math. Phys. 263 (2006), 553-581.

[Ko1] M. Kontsevich, Feynman diagrams and low-dimensional topology. In: First European Congress of Mathematics (Paris, 1992), Vol. II, Progr. in Math. 120, Birkha user, Basel, 1994, pp. 97121.

[Ko2] M. Kontsevich, Deformation quantization of Poisson manifolds, Lett. Math. Phys. 66 (2003), 157-216.

[Ko3] M. Kontsevich, Operads and motives in deformation quantization, Lett. Math. Phys. 48(1) (1999), 3572.

[KS] M. Kontsevich and Y. Soibelman, Deformations of algebras over operads and the Deligne conjecture. In Conference Moshe Flato 1999, Vol. I (Dijon), volume 21 of Math. Phys. Stud., pages 255307. Kluwer Acad. Publ., Dordrecht, 2000.

[LV] J.-L. Loday and B. Vallette, forthcoming book on operads.

[MT] D. Manchon and C. Torossian, Cohomologie tangente et cup-produit pour la quantification de Kontsevich, Ann. Math. Blaise Pascal 10 (2003), no. 1, 75106

[M] J.P. May. The Geometry of Iterated Loop Spaces, volume 271 of Lecture Notes in Mathematics. Springer-Verlag, New York, 1972.

[Me1] S.A. Merkulov, Operad of formal homogeneous spaces and Bernoulli numbers, Algebra \& Number Theory, 2 (2008), No. 4, 407-433

[Me2] S.A. Merkulov, Exotic automorphisms of the Schouten algebra of polyvector fields, preprint arXiv:0809.2385

[PT] M. Pevzner and C. Torossian, Isomorphisme de Duflo et cohomologie tangentielle, J. Geom. Phys. 51 (2004), no. $4,486505$.

[Sh1] B. Shoikhet, An $L_{\infty}$ algebra structure on polyvector fields, preprint arXiv:0805.3363, (2008).

[Sh2] B. Shoikhet, Koszul duality in deformation quantization and Tamarkins approach to Kontsevich formality, arXiv:0805.0174, to appear in Adv. Math.

[St] J.D. Stasheff. On the homotopy associativity of H-spaces, I II. Trans. Amer. Math. Soc., 108 (1963), $272-292$ \& $293-312$.

[Ta1] D.E. Tamarkin, Another proof of M. Kontsevich formality theorem, math.QA/9803025, Lett. Math. Phys. 66 (2003) 65-72.

[Ta2] D.E. Tamarkin, Action of the Grothendieck-Teichmueller group on the operad of Gerstenhaber algebras, preprint arXiv:math/0202039, 2002.

Sergei A. Merkulov: Department of Mathematics, Stockholm University, 10691 Stockholm, Sweden

E-mail address: sm@math.su.se 